\title{
On the Oscillatory Modes of Compressible Thermal Convection in inclined differentially heated cavities
}

\author{
Marcello Lappa and Tomislav Gradinscak \\ Department of Mechanical and Aerospace Engineering, University of Strathclyde \\ James Weir Building, 75 Montrose Street, Glasgow, G1 1XJ, UK \\ email: marcello.lappa@strath.ac.uk
}

\begin{abstract}
We consider the case of a tall differentially heated cavity (containing air) for which the buoyancy flow is in the so-called boundary-layer regime (displaying thin regions featured by strong temperature gradients adjacent to the vertical walls and thermally stratified fluid in the centre). By using a numerical method able to account for compressibility and variable viscosity effects, we investigate the response of such a system to the application of temperature differences in a range of characteristic numbers for which the resulting flow is expected to be time-periodic or highly timedependent. More precisely, the problem is examined by allowing both the Rayleigh number and the inclination $(\theta)$ of this configuration with respect to the vertical direction to span relatively wide intervals $\left(1.8 \times 10^{5} \leq \mathrm{Ra} \leq 3 \times 10^{6},-90^{\circ} \leq \theta \leq 90^{\circ}\right)$. Insights into the influence of variable thermodynamic properties are obtained by comparing such numerical results with equivalent simulations based on the classical Boussinesq approximation. The evolution of the considered system towards a fully turbulent state is also discussed to a certain extent.
\end{abstract}

\section{Introduction}

Thermal convection in differentially heated cavities has been a research subject for many decades. Studying the phenomena relating to convective flows inside closed geometries can provide valuable information on the fundamental mechanisms that drive a fluid system from an initially quiescent state to the onset of turbulence. The path taken by the system during this process is strongly linked to the sequence of bifurcations that it undergoes when the temperature difference is progressively increased. In turn, this evolution is extremely sensitive to the physical properties of the fluid. NonBoussinesq (NOB) effects can arise from compressibility and alterations of thermal conductivity or viscosity induced by temperature. Even minute variations in such properties can lead to remarkable changes in the fluid-dynamic instabilities displayed by the flow.

The problem is made even more complex by the sensitivity of such systems to the effective relative direction of gravity and applied temperature difference. Indeed, the hierarchy of bifurcations can be modified significantly (and dramatically in some circumstances) according to whether gravity and the applied temperature gradient are perpendicular, have the same direction or form an arbitrary angle.

It is a well-known fact that the simplest condition is that corresponding to a fluid being uniformly heated from above and cooled from below for which no convection is produced (system sticking to its initial quiescent conditions). The opposite limiting situation is that in which the direction of the temperature gradient is reversed (i.e. gravity and $\Delta \mathrm{T}$ are concurrent). While for such circumstances motion ensues with the onset of an instability only when $\Delta \mathrm{T}$ exceeds a given value (classical Rayleigh-Bénard convection), overcoming a threshold is no longer required if the externally 
imposed $\Delta \mathrm{T}$ yields temperature gradients that are primarily horizontal. In these cases motion occurs for any value of $\Delta \mathrm{T}$. Remarkably, such differences are not limited to the primary flow; the secondary, tertiary and high-order modes of convection emerging when the temperature difference is further increased do also depend significantly on the specific circumstances considered.

Such problems are made even more complex by the simple realization that such fundamental cases (with purely vertical or horizontal temperature differences) are idealized situations. Proper analysis of the rich and wide-ranging phenomena displayed by many effective natural and technological processes would require more general and realistic models or configurations, a first step along these lines being represented by the consideration of "inclined" systems (Nield [1]; Delgado-Buscalioni et al. [2]; Delgado-Buscalioni and Crespo del Arco [3,4]).

When the angle $\theta$ between the horizontal direction and the applied temperature difference is varied in the range $-90^{\circ} \leq \theta \leq 90^{\circ}$, a variety of new states become possible. Though much work over recent years has been devoted to classify and characterize the properties of all these solutions, this field continues to burgeon and bring surprises to this day even if related studies are carried out under the assumption of incompressible flow (Boussinesq approximation) and constant fluid properties (constant viscosity and thermal diffusivity).

The present work examines the consequence of this specific long-held assumption/weakness of current models, which appears not to have been questioned yet in the literature. Indeed, in the present study we target regimes of buoyancy convection produced when the inclination angle and the magnitude of the driving force (buoyancy) are changed by expressly including NOB effects.

Clarifying this issue would substantially enhance our fundamental understanding of a large number of key issues and problems, which remain unresolved and still elude us whilst being relevant to a number of both industrial and natural contexts (some of which are mentioned in the following).

Typical engineering applications include furnace engineering (Baltasar et al. [5]), the cooling of high-power devices, solar energy and nuclear power plants (von Backström and Gannon [6]; Elmo and Cioni [7]; Hu et al. [8]; Martineau et al. [9]; Bensaci et al. [10]). Additional relevance is provided by the closeness of these themes to some meteorological and geophysical problems as well as to some fundamental questions to be addressed in the general field relating to the analysis of the dynamics of non-linear systems. Apart from being well suited for the study of buoyancy and shear flow driven instabilities in the Earth's atmosphere (Lipps [11]; Thorpe [12]), differentially heated cavities may also serve as a paradigm for anisotropic pattern forming phenomena (Lappa [13]).

In the present work, we focus on the case of an elongated rectangular enclosure uniformly heated along its longest wall, cooled at the opposite side and with adiabatic conditions on the remaining boundaries. While orienting the cavity in order to make its longest wall the bottom horizontal boundary would make this a classical example of a Rayleigh-Bénard (RB) pattern forming system (mimicking to a certain extent an infinite horizontal layer), rotating it by $90^{\circ}$ would lead to the classical case of a differentially heated tall cavity for which the flow is expected to be in the socalled boundary-layer regime. When such condition is attained (typically for relatively high values 
of the Rayleigh number), thin boundary layers are formed along the lateral walls, whereas in the central core the temperature is uniform in horizontal planes and increases in the vertical direction. Interesting stability analyses based on the assumption of a basic temperature field such as that described above were originally carried out under the assumption of incompressible flow by Vest and Arpaci [14], Mizushima and Gotoh [15] and Bergholz [16]. The latter author, in particular, considered widely varying levels of stable background stratification for Pr ranging from 0.73 to $10^{3}$. Most of the available experimental results dealing with boundary-layer instabilities in tall slots have been produced for transparent liquids (see, e.g. Elder [17]; Seki et al. [18]; Chen and Thangam [19]; Chen and $\mathrm{Wu}[20]$; Wakitani [21]).

Our article concentrates on building upon, but also seeks to extend, the valuable research that has been carried out most recently on such topics with special attention to the case of gases (for which variable-density effects are expected to play a significant role). Along these lines, for instance, it is worth citing Roux et al. [22] and Lee and Korpela [23], who observed numerically steady multicellular flow formed as secondary cells under the classical assumption of constant fluid properties. At higher values of the Rayleigh number, several authors have confirmed the existence of a reverse transition from multicellular to unicellular structure (Roux et al. [22] and Chenoweth and Paolucci [24]; Brenier et al. [25]) as well as several branches of solutions characterized by different numbers of cells (Le Quéré [26] and Wakitani [27]). Unsteady multicellular solutions were investigated by Wakitani [28], while the interested reader may consult Christon et al. [29] for a summary of the results from a special session dedicated to understanding the fluid dynamics of the 8:1 thermally driven cavity which was held at the First MIT Conference on Computational Fluid and Solid Dynamics in June, 2001. Interesting numerical results about NOB effects can be found in the works by Le Quéré et al. [30], Weisman et al. [31], Bouloumou et al. [32].

For the specific case of inclined configurations and incompressible flow, some glimpses into the astonishing variety of new phenomena enabled would follow naturally from careful readings of the recent and excellent papers by Ortiz-Pérez and Dávalos-Orozco [33,34], Saury et al. [35], Torres et al. [36], Williamson et al. [37].

Though such valuable efforts and related exciting discoveries (the observed suppression of flow multiplicity, the activation of new codimension-two points and the transition to multi-modal flow, just to cite a few), however, fundamental questions persist regarding the combined role of the inclination and NOB effects on such regimes. Despite its perceived practical importance, the problem has not been investigated sufficiently. Among other things, from a more academic standpoint (beyond practical applications), the work set forth in this paper might also be regarded as a specific attempt to bridge theoretically two lines of inquiry previously running in parallel in the literature, namely that devoted to the assessment of NOB effects in horizontal or vertical rectangular cavities (Lappa [38] and references therein) and that focusing on the influence of arbitrary angles of inclination on the emerging dynamics. 


\section{Mathematical model}

\section{$\underline{2.1 \text { Geometry }}$}

The geometry that we consider in the present work is a classic two-dimensional (2D) tall vertical cavity, already used in past fundamental studies on this subject. This geometry is depicted in Fig. 1a with regards to its 'basic configuration' or orientation, namely a vertically elongated rectangular enclosure with height $\mathrm{H}$ and width $\mathrm{W}$, respectively. The heated side is on the right of the geometry and the cooled side on the left with thermally insulated horizontal adiabatic walls. Gravity $g$ is oriented in the negative $y$-direction. Inclined cases are obtained by tilting the basic configuration with respect to the vertical direction as shown in Fig. $1 \mathrm{~b}$.

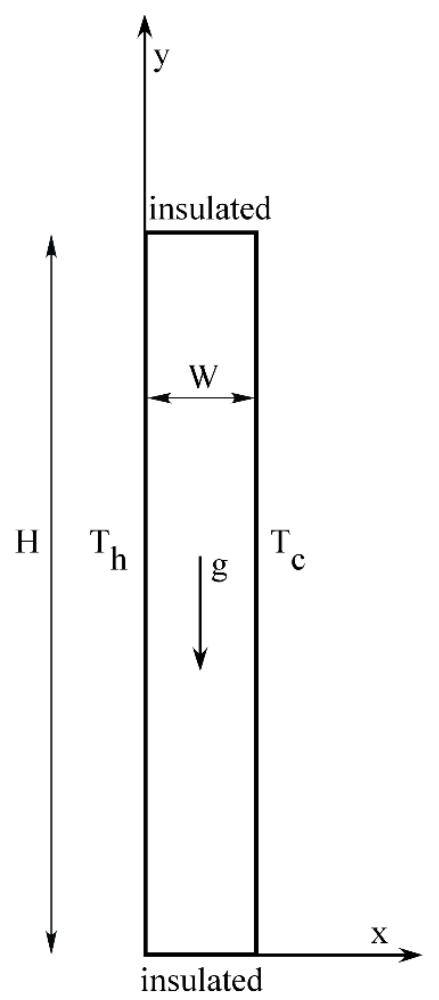

a) Basic configuration

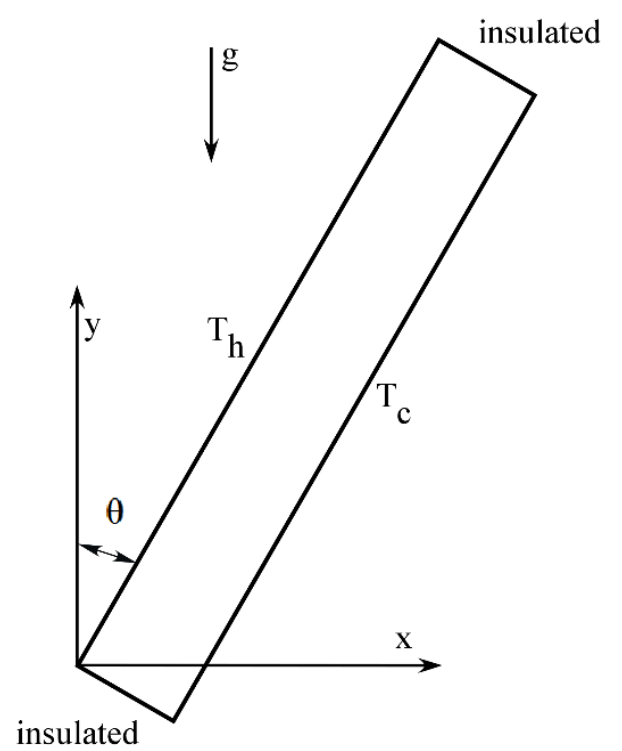

b) Inclined cavity

Fig. 1: Sketch of the differentially heated cavity.

For the situations in which we assume the flow to be incompressible, the temperature difference is supposed to be sufficiently small to satisfy the conditions required for the validity of the standard Oberbeck-Boussinesq (OB) approximation (Oberbeck [39]; Boussinesq [40]; Gray and Giorgini [41]). As explained in the introduction, the present investigation also considers other circumstances for which the temperature difference is so high that large density variations are produced that can no longer be handled in the framework of the classical Boussinesq model. For such cases, as the considered fluid is a gas, we assume molecular transport variables such as fluid viscosity and thermal conductivity to vary according to the Sutherland law. 


\section{Nomenclature}

A cavity aspect ratio $(\mathrm{H} / \mathrm{W})$

C specific heat

$f \quad$ frequency

g gravitational acceleration

$\mathrm{H} \quad$ height of the cavity

h enthalpy

$\mathrm{k}$ thermal conductivity

$\mathrm{N}$ number of grid points

$\mathrm{Nu} \quad$ Nusselt number

$\mathrm{p}$ pressure

Pr Prandtl number, $v / \alpha$

R gas constant

Ra Rayleigh number, $g \beta \Delta \mathrm{TW}^{3} / v \alpha$

$\mathrm{S}$ Constant appearing in the Sutherlands law

$\mathrm{t}$ time

T Temperature

$\mathrm{u}, \mathrm{V}$ velocity components

V fluid velocity

W cavity width

$\mathrm{x}, \mathrm{y} \quad$ Cartesian coordinates

\section{Greeks}

$\alpha \quad$ thermal diffusivity

$\beta \quad$ thermal expansion coefficient

$\Delta \quad$ increment

$\delta \quad$ increment

$\eta \quad$ Kolmogorov length scale

$\lambda \quad$ thermal boundary layer thickness

$\mu \quad$ dynamic viscosity

$v \quad$ kinematic viscosity

$\rho$ density

$\theta \quad$ inclination angle with respect to the vertical direction

Subscripts and superscripts

$0 \quad$ initial conditions

C cold

$\mathrm{H}$ hot

med average

ref reference quantity

std standard 
As a control parameter for the analysis of the stability of thermal convections it is common practice to introduce the dimensionless Rayleigh number Ra. Although there are several possible variants of such a parameter that are equally relevant, we adopt the following expression:

$R a=\frac{g \Delta T W^{3}}{T_{r e f} \nu_{r e f} \alpha_{r e f}}$

where $T_{\text {ref }}=300 \mathrm{~K}$ is a reference temperature calculated as $T_{\text {ref }}=\left(T_{\mathrm{H}}+T_{\mathrm{C}}\right) / 2 . \Delta T=T_{\mathrm{H}}-T_{\mathrm{C}}$ is the temperature difference responsible for the onset of convection. Additional useful dimensionless quantities are the nondimensional temperature difference $\varepsilon=\left(T_{\mathrm{H}}-T_{\mathrm{C}}\right) /\left(2 T_{\text {ref }}\right)$ and the reference Prandtl number, classically defined as the ratio of the reference fluid kinematic viscosity and thermal diffusivity. Since air is chosen as the working fluid, the reference Prandtl number is set to $\operatorname{Pr}=0.71$. The reference values for the dynamic viscosity $\mu_{\text {ref }}$ and thermal diffusivity $\alpha_{\text {ref }}$ are defined according to the relationships:

$\mu_{\text {ref }}=v_{\text {ref }} \rho_{\text {ref }}$

$\alpha_{r e f}=\frac{v_{r e f}}{\operatorname{Pr}}$

where density $\rho_{\text {ref }}=1.1768 \mathrm{~kg} / \mathrm{m}^{3}$ and kinematic viscosity $v_{\text {ref }}=15.68 \times 10^{-6} \mathrm{~m}^{-2} \cdot \mathrm{s}^{-1}$ are calculated for the reference temperature $T_{\text {ref. }} R_{\text {gas }}$ is the individual gas constant for air, which equals to $R_{\text {gas }}=287$ $\mathrm{J} /(\mathrm{K} \cdot \mathrm{kg})$.

In line with our choice of characteristic length used for the definition of the Rayleigh number (W), all frequencies reported in this work are made nondimensional by using $\alpha_{\text {ref }} / \mathrm{W}^{2}$ as the reference frequency. From a fundamental-research point of view, the inclined configuration can effectively be used as a paradigm system for the study of several types of instabilities and their corresponding interactions. It will be shown here how, in transitioning from the canonical RB problem $\left(\theta=-90^{\circ}\right)$ to the classical flow in heated-from-the-side systems $\left(\theta=0^{\circ}\right)$, new types of instabilities and patterns appear if NOB effects are taken into account.

\subsection{Model equations for the incompressible flow case}

Assuming that the density becomes a temperature dependent variable solely for what concerns the production term of the momentum equation and that all the other thermodynamic properties are constant, the mathematical model reduces to the standard OB approximation. Such a model consists of the simplified (incompressible formulation) Navier-Stokes and energy equation, which in dimensional form read: 
$\underline{\nabla} \cdot \underline{\mathrm{V}}=0$

$\frac{\partial \underline{V}}{\partial t}+\underline{\nabla} \cdot[\underline{V} \underline{V}]=-\frac{1}{\rho} \underline{\nabla} p+v_{r e f} \nabla^{2} \underline{V}+\underline{g}\left[1-\beta\left(T-T_{0}\right)\right]$

$\frac{\partial T}{\partial t}+\underline{\nabla} \cdot[\underline{V} T]=\alpha_{r e f} \nabla^{2} T$

where $\underline{\mathrm{V}}$ and $\mathrm{p}$ are the velocity and pressure, respectively, and $\beta$ is the thermal expansion coefficient $\beta=\partial \rho / \partial \mathrm{T}=$ const.

\subsection{Model equations for the variable-density case}

When the incompressibility assumption is no longer valid, the Navier-Stokes and energy equations must be solved in their variable-density formulation (the interested reader being referred to Paolucci [42]; Chenoweth and Paolucci [24]; Müller [43]; Munz et al. [44]; Beccantini et al. [45]; Benteboula and Lauriat [46] for further details on the derivation of such a set of equations and the related so-called low-Mach number approximation). The resulting coupled system of equations can be summarized as:

$\frac{\partial \rho}{\partial t}+\underline{\nabla} \cdot[\rho \underline{V}]=0$

$\frac{\partial \rho \underline{V}}{\partial}+\underline{\nabla} \cdot[\rho \underline{V} \underline{V}]=-\underline{\nabla} p+\underline{\nabla} \cdot[\mu \underline{\nabla} \underline{V}]+\underline{\rho g}$

$\frac{\partial \rho h}{\partial t}+\underline{\nabla} \cdot[\rho \underline{V h}]-\underline{\nabla} \cdot[\alpha \underline{\nabla h}]=\frac{D p}{D t}$

$\rho=\frac{p}{R_{\text {gas }} T}$

where $\mathrm{h}$ is the specific enthalpy.

\section{Numerical aspects}

\subsection{The method}

As previously shown in Fig. 1, the governing equations have been solved numerically assuming conditions of prescribed temperature along two parallel boundaries (the longest walls delimiting the cavity) and adiabatic conditions for the remaining boundaries (no slip conditions being considered for all the walls). In particular, we solved the balance equations and related boundary conditions in their complete non-linear and time-dependent form using the open source software OpenFoam. The 
related solution strategy relies on a classical Finite Volume Method (FVM) approach by which the governing equation are properly discretized over a finite set of control volumes with a nonstaggered variable arrangement for the different problem quantities (which means all primitive variables occupy the same computational points). The related method pertains to the so-called category of fractional-step methods or pressure-correction methods (often simply referred to as primitive-variables approach). The velocity and pressure fields are typically treated in a sequential way, with the pressure determined by using a specific pressure-correction equation. This additional elliptic equation is built by forcing the discrete momentum equation into the continuity equation. This specific modus operandi is the basis of the so-called PISO approach. For additional information, the reader should refer to Jang et al. [47] or to the exhaustive book by Moukalled et al. [48] . Because of the non-staggered collocation of variables on the grid, the related solution also relies on the well-known Rhie and Chow [49] interpolation stencil, by which it is possible to prevent the algorithm from developing spurious oscillations due to unresolved coupling between velocity and pressure (Choi et al. [50,51] and references therein).

Spatial discretization of convective terms in the momentum and energy equations has been implemented via a third order accurate Quick (Quadratic Upstream Interpolation for Convective Kinematics) scheme, while diffusive terms have been treated using the Gauss approximation and linear interpolation throughout all simulations. To account for temporal changes, time derivatives have been discretized with implicit, first order accurate, backward stepping. Finally, the pressure (elliptic) equation has been integrated using a standard conjugate gradient method or a multigrid approach.

Though the above descriptions apply to both the incompressible and variable-density formulation of the Navier-Stokes equations, the latter case deserves some additional discussion given the inherent higher complexity and increased number of equations:

The perfect gas equation of state (10) is used in order to evaluate the initial density field $\rho$. Enthalpy $\mathrm{h}$ is subsequently computed from the temperature field under the assumption of constant specific heat $\mathrm{C}_{\mathrm{p}}$. At each time step, the newly calculated enthalpy field and constant specific heat are, in turn, used to update the temperature field. The details about the time-marching algorithm can be summarised as follows:

a) solve equation (7);

b) solve a modified version of equation (8) if the momentum predictor is solved;

c) solve equation (9) ;

d) update the temperature field using the equation $\mathrm{T}=\mathrm{h} / \mathrm{Cp}$;

e) enter the PISO algorithm;

f) update the density field using equation (10).

In order to compute temperature-dependent transport properties, the dynamic viscosity $\mu$ and thermal diffusivity $\alpha$ as provided by the Sutherland's law are evaluated as follows: 


$$
\begin{aligned}
\mu & =\frac{S \sqrt{T}}{1+T_{s} / T} \\
\alpha & =\frac{\mu C_{v}}{\widetilde{C}_{p}}\left(1.32+1.77 \frac{R_{\text {gas }}}{C_{v}}\right)
\end{aligned}
$$

where $S$ and $T_{\mathrm{s}}$ are known constants with values $S=1.4584 \cdot 10^{-6} \mathrm{~kg} /\left(\mathrm{m} \cdot \mathrm{s} \cdot \mathrm{K}^{1 / 2}\right)$ and $T_{\mathrm{s}}=110.33 \mathrm{~K}$ and $\widetilde{C}_{p}$ in eq. (12) is calculated according to:

$$
\widetilde{C}_{p}=\frac{\delta T\left(h-h_{T_{s t d}}\right)+C_{p}}{\sqrt{\delta T}+1}
$$

where $\delta T=\left(T-T_{\text {std }}\right)$ and $T_{\text {std }}$ is the standard temperature $T_{\text {std }}=293 \mathrm{~K}$.

\subsection{Initial and boundary conditions}

For the convenience of the reader, the initial and boundary conditions are reported in Tables I and II, respectively.

Table I: Initial conditions for incompressible and compressible flow.

\begin{tabular}{lccc}
\hline \hline Variable & $T[\mathrm{~K}]$ & $p[\mathrm{pa}]$ & $\underline{\mathrm{V}}[\mathrm{m} / \mathrm{s}]$ \\
Boussinesq flow & 300 & 101325 & 0 \\
Variable-properties flow & 300 & 101325 & 0 \\
\hline \hline
\end{tabular}

Table II: Boundary conditions for incompressible and compressible flow (n denotes the direction perpendicular to the wall).

\begin{tabular}{lccc}
\hline \hline Variable & \multicolumn{1}{c}{$p$} & $\underline{\mathrm{V}}$ \\
\hline \hline Left wall & Incompressible flow with OB approximation & \\
Right wall & $T_{\mathrm{H}}=303 \mathrm{~K}$ & $\underline{\nabla} p=\underline{\rho g} \cdot \underline{n}$ & $\underline{\mathrm{V}}=0 \mathrm{~m} / \mathrm{s}$ \\
$T_{\mathrm{C}}=297 \mathrm{~K}$ & $\underline{\nabla p}=\underline{\rho g} \cdot \underline{n}$ & $\underline{\mathrm{V}}=0 \mathrm{~m} / \mathrm{s}$ \\
Top wall & $\frac{\partial T}{\partial n}=0$ & $\underline{\nabla} p=\underline{\rho g} \cdot \underline{n}$ & $\underline{\mathrm{V}}=0 \mathrm{~m} / \mathrm{s}$ \\
Bottom wall & $\frac{\partial T}{\partial n}=0$ & $\underline{\nabla} p=\underline{\rho g} \cdot \underline{n}$ & $\underline{\mathrm{V}}=0 \mathrm{~m} / \mathrm{s}$ \\
Left wall & $T_{\mathrm{H}}=480 \mathrm{~K}$ & $\underline{\nabla} p=\underline{\rho g} \cdot \underline{n}$ & $\underline{\mathrm{V}}=0 \mathrm{~m} / \mathrm{s}$ \\
Right wall & $T_{\mathrm{C}}=120 \mathrm{~K}$ & $\underline{\nabla} p=\underline{\rho g} \cdot \underline{n}$ & $\underline{\mathrm{V}}=0 \mathrm{~m} / \mathrm{s}$ \\
Top wall & $\frac{\partial T}{\partial n}=0$ & $\underline{\nabla} p=\underline{\rho g} \cdot \underline{n}$ & $\underline{\mathrm{V}}=0 \mathrm{~m} / \mathrm{s}$ \\
Bottom wall & $\frac{\partial T}{\partial n}=0$ & $\underline{\nabla} p=\underline{\rho g} \cdot \underline{n}$ & $\underline{\mathrm{V}}=0 \mathrm{~m} / \mathrm{s}$ \\
\hline
\end{tabular}




\subsection{Benchmark and mesh independence analyses}

Buoyancy flow in square cavity

Table III illustrates the comparison between the results obtained using OpenFOAM (present work) and those reported by other authors [52-55] with regard to the classical square-cavity benchmark case for $\mathrm{Ra}=10^{6}$. As a sensitive parameter for comparison we use the mean value of the Nusselt number $\mathrm{Nu}$ on the hot and cold walls. It can be seen that the results agree with those in the abovementioned studies to less than $0.5 \%$ for both the variable-property and incompressible (OB) cases.

\section{Differentially heated tall cavity}

In order to make sure that conditions of mesh independence are attained when the tall cavity case is considered (aspect ratio height/width $\mathrm{A}=8$ ), we analysed three different grids assuming the classical configuration $\theta=0^{\circ}$, i.e. walls oriented vertically and the horizontal temperature difference, already considered in past studies ([32]). The effects produced by increasing the number of points (evenly spaced along the horizontal and vertical directions) for the variable-property case with $\mathrm{Ra}=3.4 \times 10^{5}$, namely $(60 \times 700),(70 \times 800)$ and $(75 \times 900)$, are shown in Table IV (note that, unless otherwise specified, hereafter, the denominations of "compressible" and "incompressible" flow will be used as synonyms for the variable-property and constant-property models, respectively).

Table III: Benchmark analysis (data considered: air, $\mathrm{T}_{\text {med }}=\mathrm{T}_{0}=600 \mathrm{~K}, \mathrm{p}_{0}=101325 \mathrm{~Pa}, \mathrm{Pr}=0.71$, $\mathrm{A}=1, \theta=0^{\circ}, \mathrm{Ra}=10^{6}, 250 \times 250$ grid points)

\begin{tabular}{lcc}
\hline \hline & $\mathrm{Nu}_{\text {cold }}$ & $\mathrm{Nu}_{\text {hot }}$ \\
\hline \hline & Compressible flow (Sutherland law) & \\
Present & 8.73403 & 8.69183 \\
Reference & 8.7135 & 8.7160 \\
Error (\%) & 0.24 & -0.28 \\
& Compressible flow ( $\mu$ const.) & \\
Present & 8.82213 & 8.81913 \\
Reference & 8.8133 & 8.8112 \\
Error (\%) & 0.10 & 0.09 \\
& Incompressible flow & \\
Present & 8.81733 & 8.81733 \\
Reference & 8.8133 & 8.8112 \\
Error (\%) & 0.04 & 0.07 \\
\hline \hline
\end{tabular}


Table IV: Mesh independence analysis (data considered: air, $\mathrm{T}_{\text {med }}=\mathrm{T}_{0}=300 \mathrm{~K}, \mathrm{p}_{0}=101325 \mathrm{~Pa}, \mathrm{Pr}$ $=0.71, \mathrm{~A}=8, \theta=0^{\circ}, \mathrm{Ra}=3.4 \times 10^{5}$, Compressible flow $(\mu=$ const. $)$ )

\begin{tabular}{lcc}
\hline \hline $\mathrm{N}_{\mathrm{x}} \times \mathrm{N}_{\mathrm{y}}$ & $\begin{array}{c}\text { Nondimensional } \\
\text { frequency }\end{array}$ & $(\%)$ variation \\
\hline \hline $60 \times 700$ & 146.98 & - \\
$70 \times 800$ & 148.02 & $<1 \%$ \\
$75 \times 900$ & 149.07 & $<1 \%$ \\
\hline \hline
\end{tabular}

Though conditions of grid-independence could be assumed for a mesh (60x700), we decided to conduct all the simulations in this work using the $(75 \times 900)$ mesh, as we found it sufficiently dense to capture all the spatial scales involved in these phenomena for Ra up to $3 \times 10^{6}$ (see the related discussions in Sect. 3.4). This also guaranteed grid-independence conditions in the case in which the cavity is not vertical (e.g., when it is horizontal and heating is applied from below, i.e. $\theta=-90^{\circ}$ ). Further validation of the code was obtained by comparing the results for the tall cavity $\left(\theta=0^{\circ}\right)$ with other available results in the literature for the case of compressible flow. To do so, we had to sift through existing studies with different foci in order to identify relevant cases to be considered for comparison. In particular, by using the mesh specified above we found the flow to become oscillatory at the same critical Rayleigh number $\left(\mathrm{Ra} \cong 1.7 \times 10^{5}\right)$ determined by (Le Quéré et al. [30]) for the case of compressible flow with variable viscosity; moreover, assuming compressible flow with constant viscosity and $\mathrm{Ra}=3.4 \times 10^{5}$ we could get a satisfactory agreement in terms of nondimensional frequency with [32].

\subsection{Mesh assessment against the Kolmogorov length scale}

Given the relatively wide range of Rayleigh number values considered (up to $3 \times 10^{6}$ ), we also deemed it necessary to assess the resolution requirements in relation to the value taken by the Kolmogorov length scale $(\eta)$.

This requirement relates to the well-known property of turbulence to develop a hierarchy of scales through which the energy flows from larger scales towards smaller scales. In particular, the Kolmogorov's hypothesis postulates the existence of an "inertial" wavenumber region where the flux of the cascading quantity across any scale can be determined solely from dynamic variables on that scale (similarity hypothesis, we will come back to this concept later in this work). Such inertial range is limited from one side by the scales of the order of Kolmogorov length $\eta$ where the flow kinetic energy tends to be dissipated by viscous effects and from the other side by the length-scale of the energy-containing range (the scale $\mathrm{L}$ at which energy is injected into the fluid and funnelled into the cascade). In general, it is known that these two limiting scales at the extremes of the cascade can 
differ by several orders of magnitude at high Rayleigh numbers and that in a perfect simulation the mesh size should not be larger than the Kolmogorov scale.

Along these lines, the grid-size and the minimum spacing determined on the basis of the mesh refinement study discussed in Sect. 3.3 have also been assessed against such requirements for both limiting situations represented by $\theta=0^{\circ}$ and $\theta=-90^{\circ}$.

In the first case, some relevant information can be found in the works by Paolucci [56], Farhangnia et al. [57] and Trias et al. [58]. As a general criterion, in order to ensure that the smallest flow structures in turbulent natural convection, i.e. the Kolmogorov length and time scales, are adequately resolved for the case in which the applied temperature gradient is perpendicular to gravity, these authors proposed the following spatial and temporal spacing requirements:

$$
\begin{aligned}
& \Delta x, \Delta y \leq \eta=\pi\left(\frac{16 \operatorname{Pr}}{R a}\right)^{3 / 8} \\
& \Delta t \leq 8 \pi\left(\operatorname{Pr} R a^{3}\right)^{-1 / 4}
\end{aligned}
$$

Moreover, they found the thickness $\left(\lambda_{\theta}\right)$ of the conductive sublayer adjacent to the active (vertical) walls to scale as:

$$
\lambda_{\theta}=3.91 \operatorname{Pr}^{1 / 6}\left(\frac{R a}{2}\right)^{-1 / 3}
$$

The time step used for the present simulations was much smaller than the limit expressed by eq. (14b). Moreover, the reader will easily verify that, on increasing the Rayleigh number from the lowest $\left(1.9 \times 10^{5}\right)$ to the highest value $\left(3 \times 10^{6}\right)$ considered here, with the mesh indicated in Sect. 3.3 the extremes of the intervals covered by $\Delta x / \eta, \Delta y / \eta$, and $\Delta x / \lambda_{\theta}$ would be $(1 / 6,1 / 2),(1 / 8,2 / 7)$, and $(1 / 6,1 / 2)$, respectively, which can be considered well within the constraints prescribed by eqs. (14a) and (15).

A similar assessment for the case in which the temperature gradient is parallel to gravity $\left(\theta=-90^{\circ}\right.$ corresponding to Rayleigh-Bénard convection), has been based on equivalent criteria available in the literature (Shishkina et al., [59]). As an example, according to Kerr [60] and confirmed by De et al. [61] and Scheel et al. [62]:

$\eta=1.3 R a^{-0.32}$

Again, comparison of our maximum grid resolution to $\eta$ leads to the conclusion that our grid spacing was either smaller than or comparable to the Kolmogorov scale. Indeed, from the smallest to the highest Rayleigh number, $\Delta x / \eta$ and $\Delta y / \eta$, vary approximately from $1 / 2$ to 1 and from $1 / 3$ to 1 , respectively, which satisfies the general criteria indicated by [59]. 


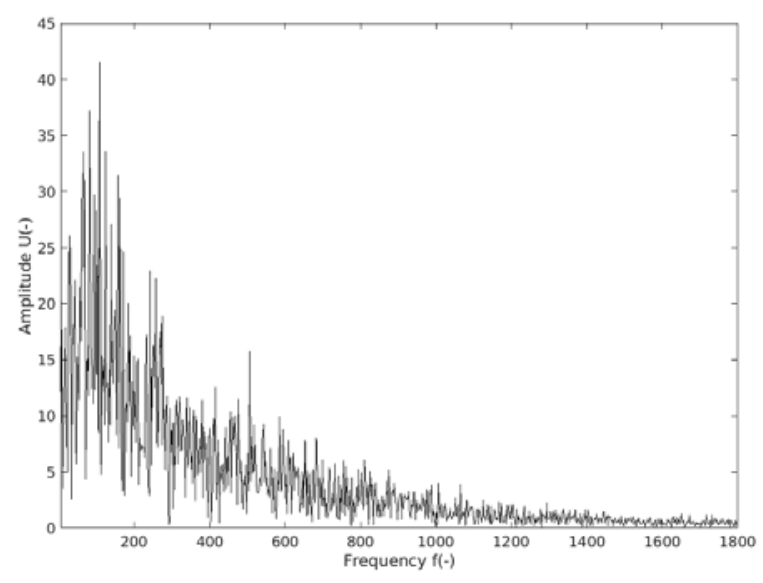

a)

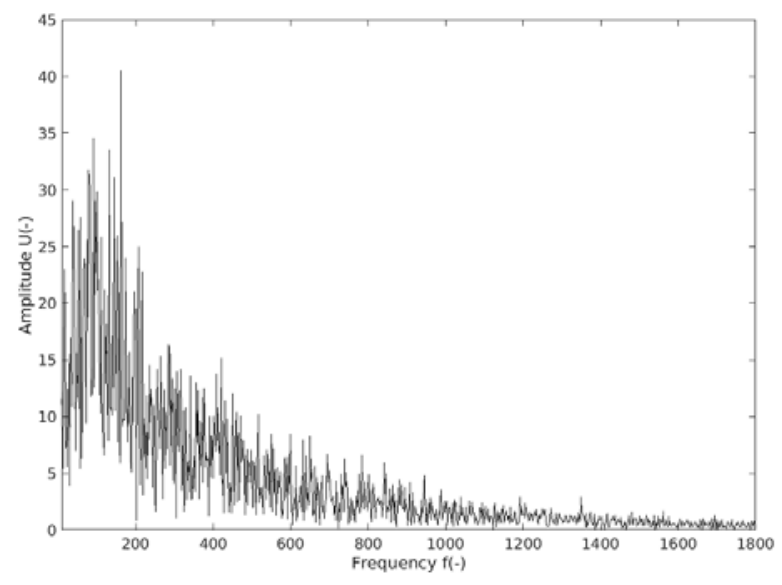

b)

Fig. 2: Frequency spectrum (data considered: air, $\mathrm{T}_{\text {med }}=\mathrm{T}_{0}=300 \mathrm{~K}, \mathrm{p}_{0}=101325 \mathrm{~Pa}, \mathrm{Pr}=0.71$, $\mathrm{A}=8, \theta=-45^{\circ}, \mathrm{Ra}=3 . \times 10^{6}, \varepsilon=0.6$, Compressible flow, variable properties): a) $65 \times 780$, b) $75 \times 900$.

Along these lines, Figure 2 finally provides evidence for the ability of the algorithm to guarantee conditions of mesh independence even if a representative inclined configuration and the "worst" conditions in terms of Rayleigh numbers are considered (namely, $\theta=-45^{\circ}$ and $\mathrm{Ra}=3 \times 10^{6}$ ). By increasing the mesh resolution from $65 \times 780$ to $75 \times 900$, no significant changes can be seen in the spectral content of the flow in terms of amplitude. Among other things, this may be regarded as a justification for our explicit intention not to implement turbulence models in our numerical framework (though many of such models could easily be accessed via the OpenFoam platform). As explained before, in general, the simulation of turbulent fluid flows is made very complex by the wide range of scales typically involved in these problems. Approximations of such flows can be achieved numerically either by solving directly the unsteady balance equations with an adequate number of grid points (DNS) or by replacing them with an equivalent set of equations (somehow accounting for turbulent effects that cannot be captured directly by the used grid). This alternative set of equations can be obtained by filtering the original equations in time and introducing a closure model for the Reynolds stresses (RANS approaches) or filtering the equations in space and introducing an adequate representation for the sub-grid dynamic stresses and heat flux (the so-called LES strategy). However, in the related "closure relations" both of these methods involve parameters/constants which need to be modified every time in order to produce accurate results. In addition, these parameters depend on many factors such as the flow regime, flow configuration, Rayleigh number etc. and may take substantially different values in space.

On the other hand, direct solution (DNS) of the governing partial differential equations (the NavierStokes equations complemented by the energy equation for the diffusive and convective transport of heat) is not free of bottlenecks. It is very demanding (often prohibitive) in terms of computational 
resources and simulation time required ([63]). As shown in Fig. 2, however, the present mesh proved sufficient to resolve all the typical spatial and temporal scales involved in the considered dynamics for Ra not exceeding $3 \times 10^{6}$, as witnessed by the negligible modifications experienced by the flow in terms of frequency spectrum (further details on such scales and the typical size of "eddies" produced by the turbulent flow will be given in Sect. 4). It cannot be excluded, of course, that values of the Rayleigh number larger than those considered here would require RANS or LES approaches such as those used by other authors (see, e.g., the relevant efforts by Altaç and Ugurlubilekc [64], Mason [65], Kimmel and Domaradzki [66] and Salat et al. [67]).

\section{Results}

Following earlier examples in the literature [32,68], we set the dimensionless temperature difference to $\varepsilon=0.6$ for compressible flows (with the temperature difference considered for incompressible flow corresponding to $\varepsilon=0.01$ ). Three values of the Rayleigh number are investigated in particular: $R a=1.9 \times 10^{5}, R a=3 \times 10^{5}$ and $R a=3 \times 10^{6}$. The inclination angle is varied between $\theta=-90^{\circ}$ and $\theta=90^{\circ}$ (with $\theta=0^{\circ}$ being the reference case as explained earlier). The initial conditions used in the computations correspond to iso-thermal fluid (at the reference temperature) at rest with $p_{0}=101325 \mathrm{~Pa}$ and $T_{0}=T_{\text {ref }}=300 \mathrm{~K}$.

\subsection{Canonical cases with vertical or horizontal cavity (Rayleigh-Bénard flow)}

Prior to addressing more complex configurations in which the cavity has an intermediate orientation in space with respect to the vertical or horizontal directions, it is instructive to consider such 'limiting' situations. This may be seen as a convenient strategy to lead the reader to the proper interpretation of multiple and variegated results via a step-by-step approach (we introduce situations of increasing complexity accordingly as the discussion progresses). Yet pursuing this approach, we begin our analysis from the simplified case in which the density and the other thermodynamic properties are constant (classical OB limit). 


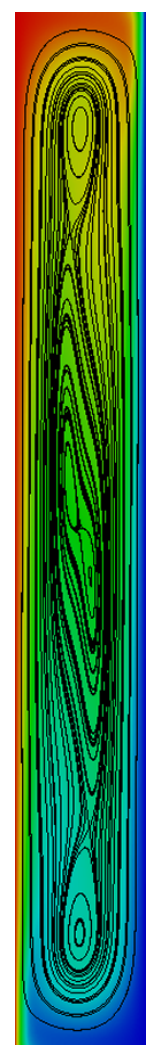

a) $R a=1.9 \times 10^{5}$

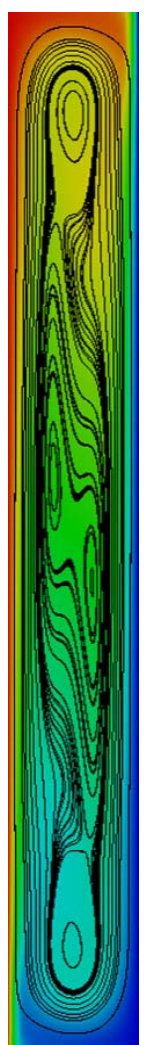

b) $R a=3 \times 10^{5}$

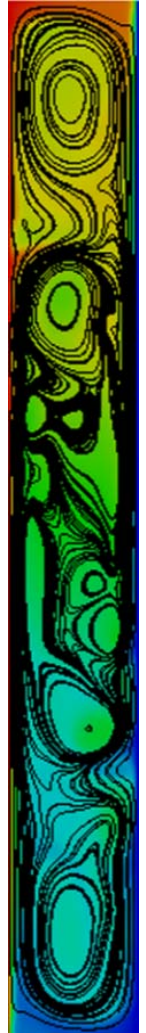

c) $R a=3 \times 10^{6}$

Fig. 3: Structure of temperature and velocity fields of incompressible flow for $\theta=0^{\circ}$.

The typical evolution of the flow established in the vertical cavity $\left(\theta=0^{\circ}\right.$ case) for increasing values of the Rayleigh number can be seen in Fig. 3. For $R a=1.9 \times 10^{5}$ the flow is steady and skewsymmetric (Fig. 3a). The related pattern may be regarded as a classical manifestation of the aforementioned boundary layer regime (thin boundary layers are clearly recognizable along the lateral walls, whereas in the central region the temperature is uniform along the horizontal direction and increases in the vertical direction).

A similar flow structure is retained for $R a=3 \times 10^{5}$ (Fig. 3b), but the flow is now oscillatory. Both flows resemble that reported by Salinger et al. [69] (though in their case the results are related to Ra $=3.61 \times 10^{5}$ ). One peculiar feature of these flows is the constant presence of two relatively large circulations (embedded in the main convective system) at the top and the bottom of the cavity (visible in both Fig. $3 a$ and $3 b$ ).

This seems to be a persistent characteristic of the flow as witnessed by the case shown in Fig. 3c. Though in this situation convection is highly oscillatory with a relatively complex frequency spectrum (see Sect. 4.2.3) and many eddies with different sizes unevenly distributed in the physical domain (which makes it essentially chaotic), two big circulations are still steadily located in proximity to the top and the bottom walls of the cavity.

Changing the orientation of the cavity from vertical to horizontal has a remarkable effect. As expected, a $90^{\circ}$ rotation causes a shift in the prevailing mode of convection, which changes from 
the boundary-layer regime discussed before to the standard Rayleigh-Bénard flow (that is obtained when a system is uniformly heated from below and cooled from above).

The consequence of an increase in the Rayleigh number is now less dramatic in terms of patterning behaviour. The most striking difference is represented by the number of vortices which is $\mathrm{m}=9$ for $R a=1.9 \times 10^{5}$ and $\mathrm{m}=8$ for $R a=3 \times 10^{5}$ (Fig. 4). Another distinguishing mark can be found in the fact that it is necessary to increase the Rayleigh number up to $R a=\mathrm{O}\left(10^{6}\right)$ to cause a transition to oscillatory flow ( $R a$ must be larger than $2 \times 10^{6}$, that is at least one order of magnitude higher than that required for the transition to unsteadiness in the $\theta=0^{\circ}$ case). As shown in Fig. $4 \mathrm{c}$, for $R a=$ $3 \times 10^{6}$, though the flow is oscillatory, the spatial structure is still somewhat regular and ordered. The number of rolls is still 8 as for $R a=3 \times 10^{5}$ and randomly distributed small-sized eddies (such as those visible in Fig. 3c) are not a feature of the flow.

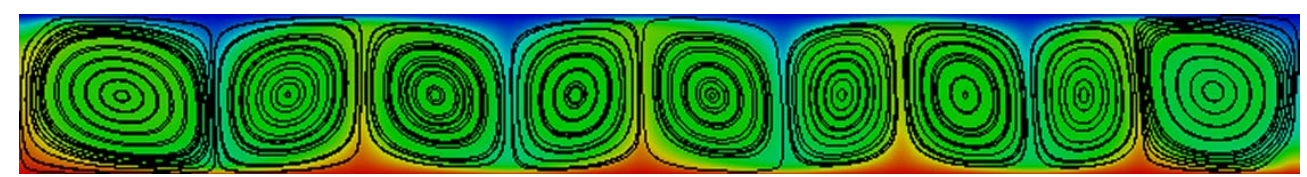

a) $R a=1.9 \times 10^{5}$

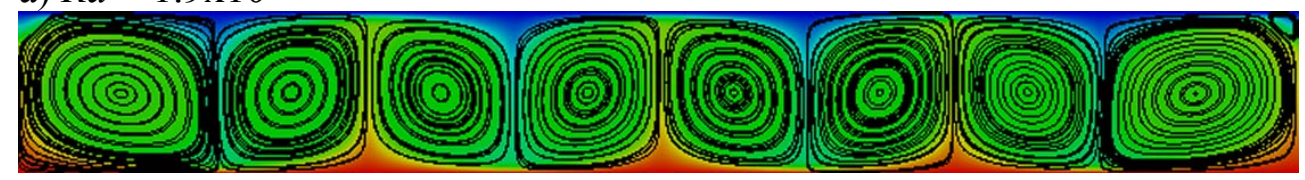

b) $R a=3 \times 10^{5}$

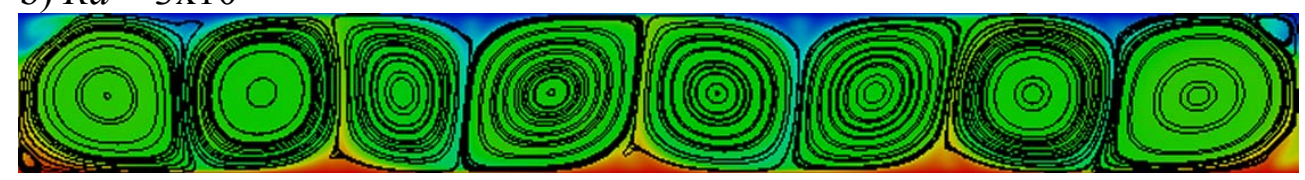

c) $R a=3 \times 10^{6}$

Fig. 4: Structure of temperature and velocity fields of incompressible flow for $\theta=-90^{\circ}$.

We can now come back to the vertical-cavity case and consider the implications and intricacies eventually brought in by NOB effects (varying density, viscosity and thermal diffusivity). One of the most interesting differences produced by such factors (for the conditions considered here) is the breaking of the skew symmetry.

Such a rupture of symmetry can be seen in both the velocity field and temperature distribution. Moreover, a stagnation area appears in the upper part of the cavity for $R a=1.9 \times 10^{5}$ and $R a=$ $3 \times 10^{5}$. The reason for this flow detachment from the walls can be found in the "conversion of kinetic energy into potential energy due to the thickening of the boundary layer on the hot side" (Bouloumou et al. [32]). Under these conditions, the flow is already time-periodic for $R a=1.9 \times 10^{5}$ while for $R a=3 \times 10^{5}$ it is oscillatory with distinct frequencies in the spectrum (we will come back to these interesting aspects later).

Some non-negligible departures from the constant-properties model can also be spotted for the case $\theta=-90^{\circ}$ (horizontal orientation and heating from below). As shown in Fig. 6, the flow patterns are 
similar to those obtained under the constraint of constant density and thermodynamic properties. Nevertheless, as expected (see, e.g., Zhang et al. [70]; Robinson and Chan [71]), some asymmetry involving the horizontal boundary layers (up-down asymmetry) is also evident in this case.

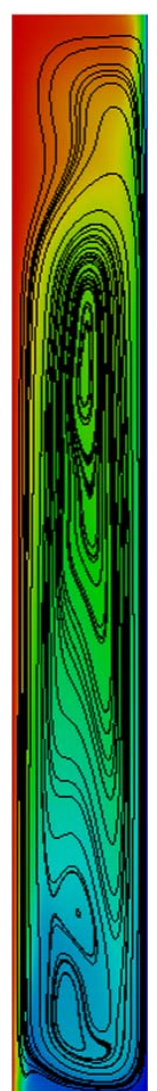

a) $R a=1.9 \times 10^{5}$

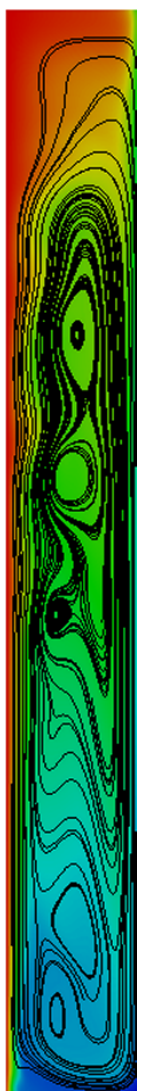

b) $R a=3 \times 10^{5}$

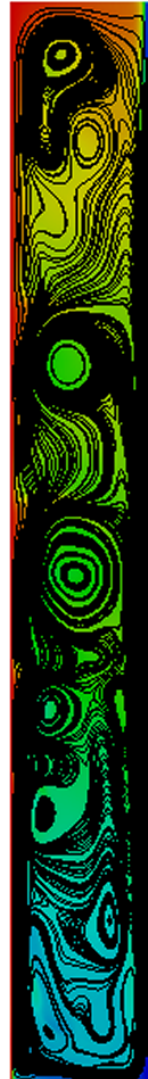

c) $R a=3 \times 10^{6}$

Fig. 5: Structure of temperature and velocity fields of compressible flow $(\varepsilon=0.6)$ for $\theta=0^{\circ}$.

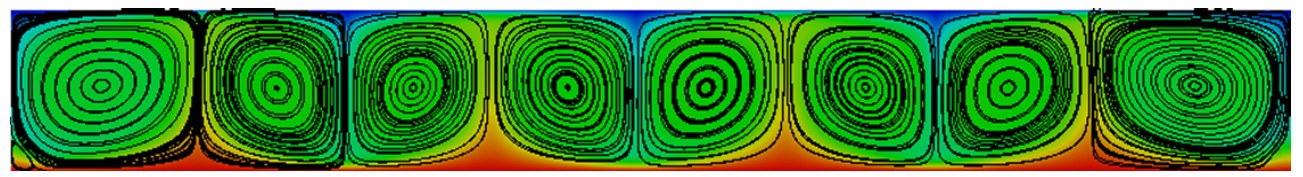

a) $R a=1.9 \times 10^{5}$

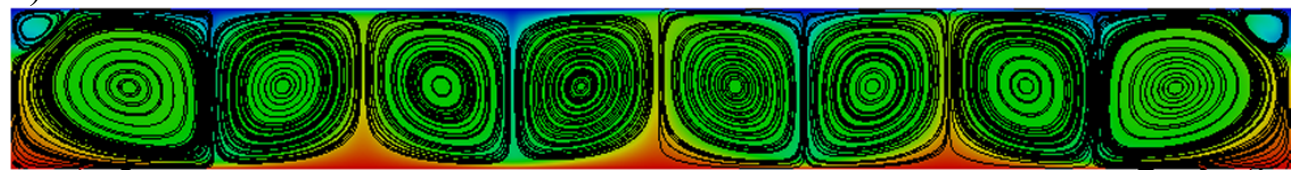

b) $R a=3 \times 10^{5}$

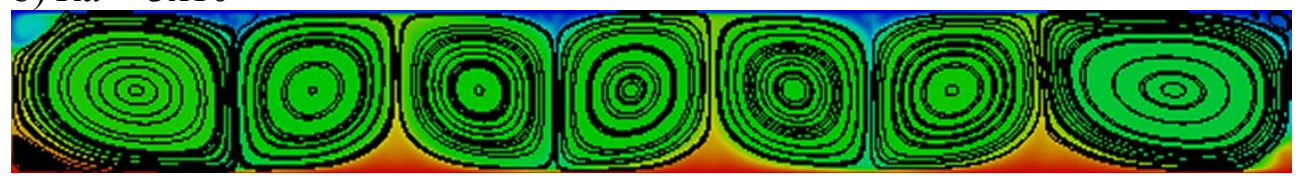

c) $R a=3 \times 10^{6}$

Fig. 6: Structure of temperature and velocity fields of compressible flow $(\varepsilon=0.6)$ for $\theta=-90^{\circ}$. 
With a behaviour relatively similar to that seen in the Boussinesq limit, for $R a=1.9 \times 10^{5}$ and $R a=$ $3 \times 10^{5}$ the variable-property flow is steady, while it is oscillatory for $R a=3 \times 10^{6}$. However, some changes can be noticed in the wavenumber, i.e. the number of rolls. This number is $\mathrm{m}=8$ and $\mathrm{m}=7$ for $R a=1.9 \times 10^{5}$ and $R a=3 \times 10^{6}$, respectively.

In this case the oscillations manifest as a rhythmic back and forth displacement (not shown) of the rolls (a snapshot can be seen in Fig. 6c).

For the convenience of the reader, the oscillatory dynamics relating to compressible flow for $\theta=0^{\circ}$ have been reported in Fig. 7 for $R a=1.9 \times 10^{5}$. It can be seen in this case that two circulations are periodically created and subsequently merged in proximity to the centre of the cavity as time passes. For $R a=3 \times 10^{5}$ similar rolls nucleate just below the middle plane of the cavity. Such circulations then travel in the positive $y$-direction and merge with the larger roll located in the upper part of the cavity (Fig. 8). Like the incompressible case, for $R a=3 \times 10^{6}$ the regime is chaotic with several new relatively small vortices and eddies being created continuously and merging without a particular order or a clearly recognizable evolutionary path.
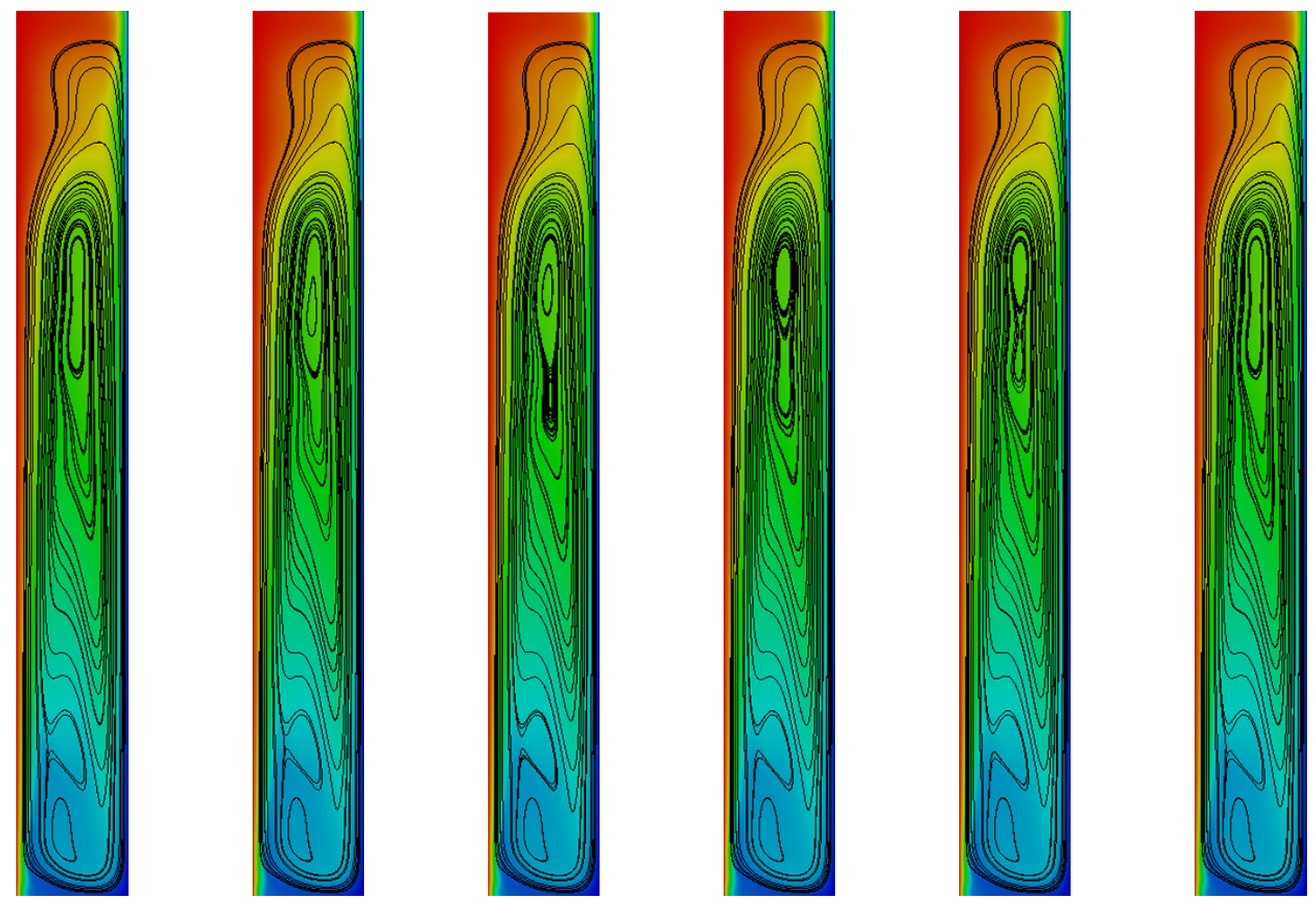

Fig. 7: Series of flow patterns (compressible flow, $\mathrm{Ra}=1.9 \times 10^{5}, \varepsilon=0.6, \theta=0^{\circ}, f=130.08$ ). 

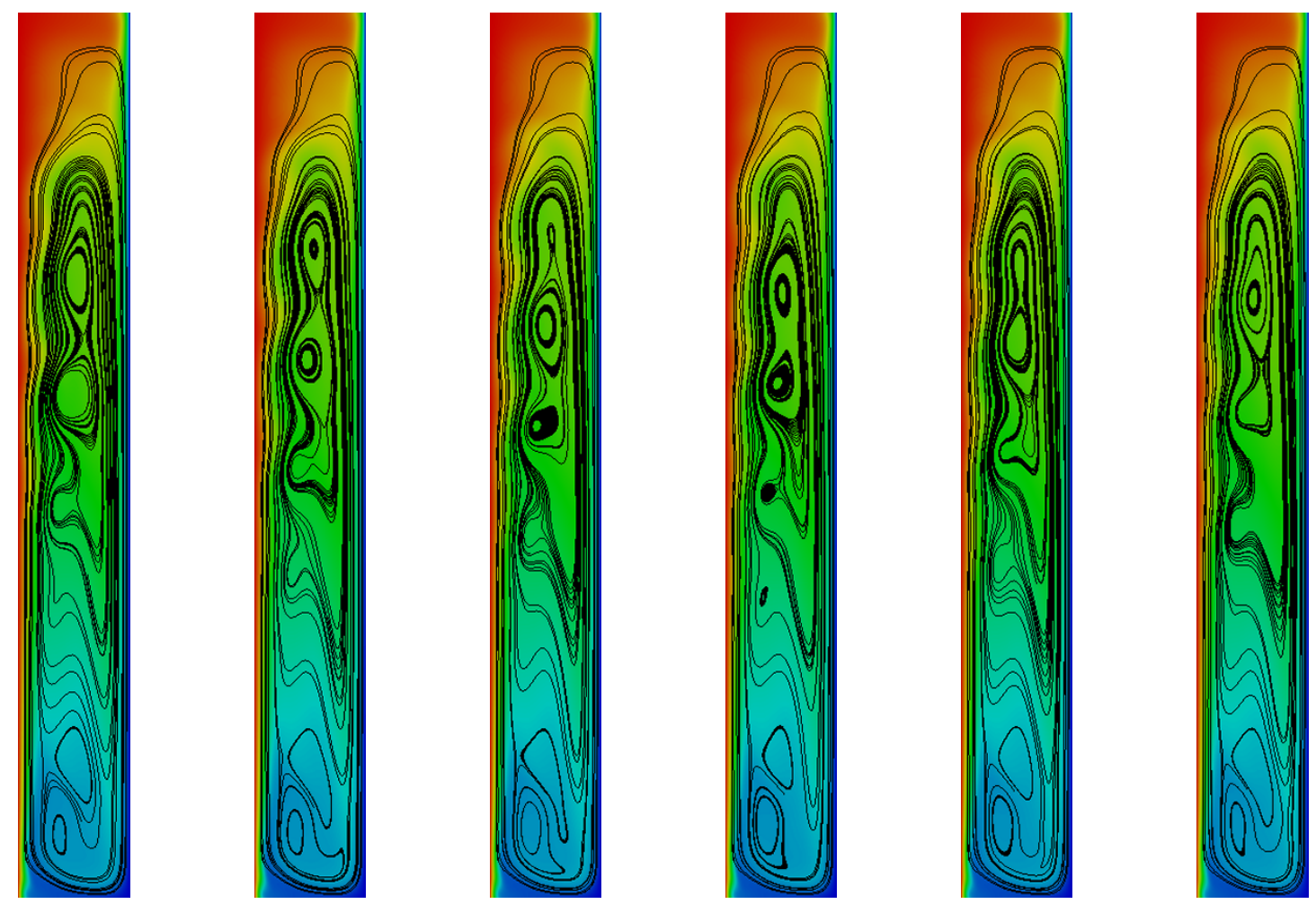

Fig. 8: Series of flow patterns (compressible flow, $\mathrm{Ra}=3 \times 10^{5}, \varepsilon=0.6, \theta=0^{\circ}, f_{D}=178.84$ ).

\subsection{Inclined configurations}

Having completed a description of the fundamental cases for which a proper interpretation can be attempted on the basis of earlier studies about classical configurations, we turn now to more complex circumstances in which the inclination angle of the cavity can be arbitrary. More specifically we allow the angle $\theta$ to span the entire interval $-90^{\circ} \leq \theta \leq 90^{\circ}$, which would lead the system from the standard $\mathrm{RB}$ convection $\left(\theta=-90^{\circ}\right)$ to the trivial situation in which no convection can emerge (cavity uniformly heated from above, $\theta=90^{\circ}$ ), passing through the aforementioned boundary-layer regime when $\theta$ is in a given (relatively limited) neighbourhood of $\theta=0^{\circ}$.

It is worth beginning the related analysis from the important observation that, after the departure of the angle from the $\theta=-90^{\circ}$ condition, the basic state is characterized not only by a linear temperature gradient but also by a symmetry-breaking shear flow (not present at the onset of standard RB convection, which evolves from an initially quiescent and patternless state). 
As shown in Fig. 1b, indeed, the component of gravity tangential to the fluid layer will cause buoyant fluid to flow up along the warm wall and down along the cold wall. This shear flow, in turn, tends to break the in-plane isotropy of the usual horizontal layer heated from below, causing a departure of the dynamics from well-known RB mechanisms (Chen and Pearlstein [72]; Fujimura and Kelly [73]; Kaloni and Qiao [74]). For $\theta<0$, the higher the absolute value of $\theta$, the less important the contribution of the shear flow is, until for $\theta=-90^{\circ}$ classical RB convection is recovered.

Given the high number of degrees of freedom displayed by the considered problem (inclination angle, Rayleigh number, incompressible or variable-properties model), we try to make the presentation (and subsequent analysis) of the results more intuitive and manageable via the introduction of condensed "maps" showing the evolution of the flow for fixed values of $\mathrm{Ra}$, different values of $\theta$ and distinct assumptions (Boussinesq vs NOB model for $\varepsilon=0.6$ ). Such maps are essentially polar diagrams where all the different solutions have been systematically reported in terms of representative snapshots (arranged as two concentric layers according to whether the incompressible or variable-density model are used, and aligned along different radial directions, each corresponding to a given value of $\theta$ ).

This specific strategy led us to cast the results into three main figures (Figs. 9, 10 and 11). As the reader will realize by inspecting such maps, each figure is complemented by qualitative descriptions of the flow behaviour and related dominant frequency $f_{D}$ (frequency with maximum amplitude in the spectrum). We recognized (and classified accordingly) 5 distinctive types of flow: steady convection, oscillatory flows with one frequency (purely periodic convection), oscillatory flows with more frequencies (multi-modal flow) and turbulent states (to be distinguished in broadband states in which a dominant frequency can still be clearly identified and fully chaotic states in which the amplitude of oscillations varies continuously with the frequency over a relatively large interval). 


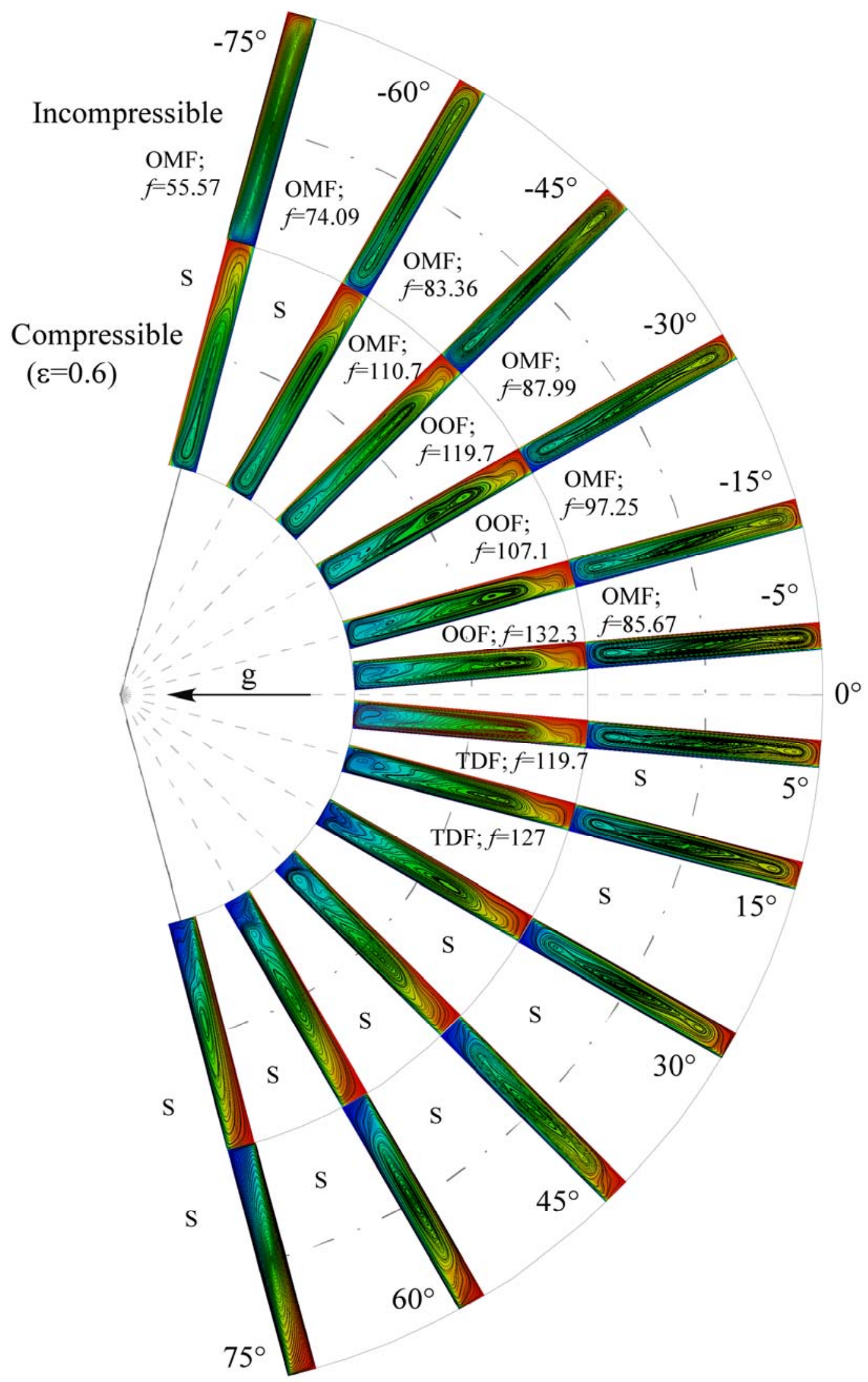

Figure 9: Map with different regimes for $\mathrm{Ra}=1.9 \times 10^{5}$. Legend: $\mathrm{S}-$ Steady; $\mathrm{T}-$ Turbulent; OOF oscillatory flow with on frequency; OMF - oscillatory flow with more frequencies; TDF Turbulent flow with one dominant frequency. 


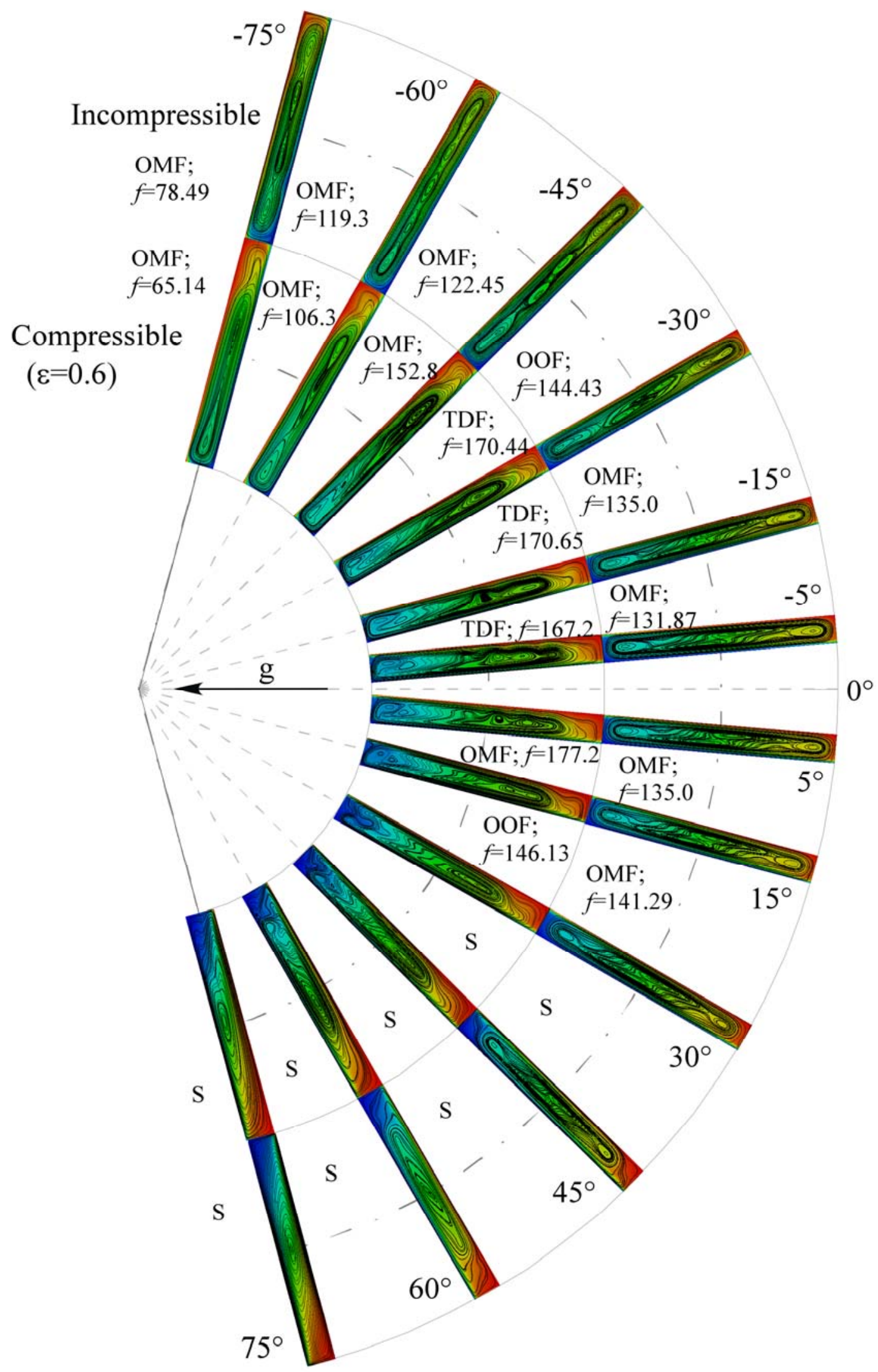

Figure 10: Map with different regimes for $\mathrm{Ra}=3 \times 10^{5}$. Legend: $\mathrm{S}-\mathrm{Steady} ; \mathrm{T}-$ Turbulent; OOF oscillatory flow with on frequency; OMF - oscillatory flow with more frequencies; TDF Turbulent flow with one dominant frequency. 


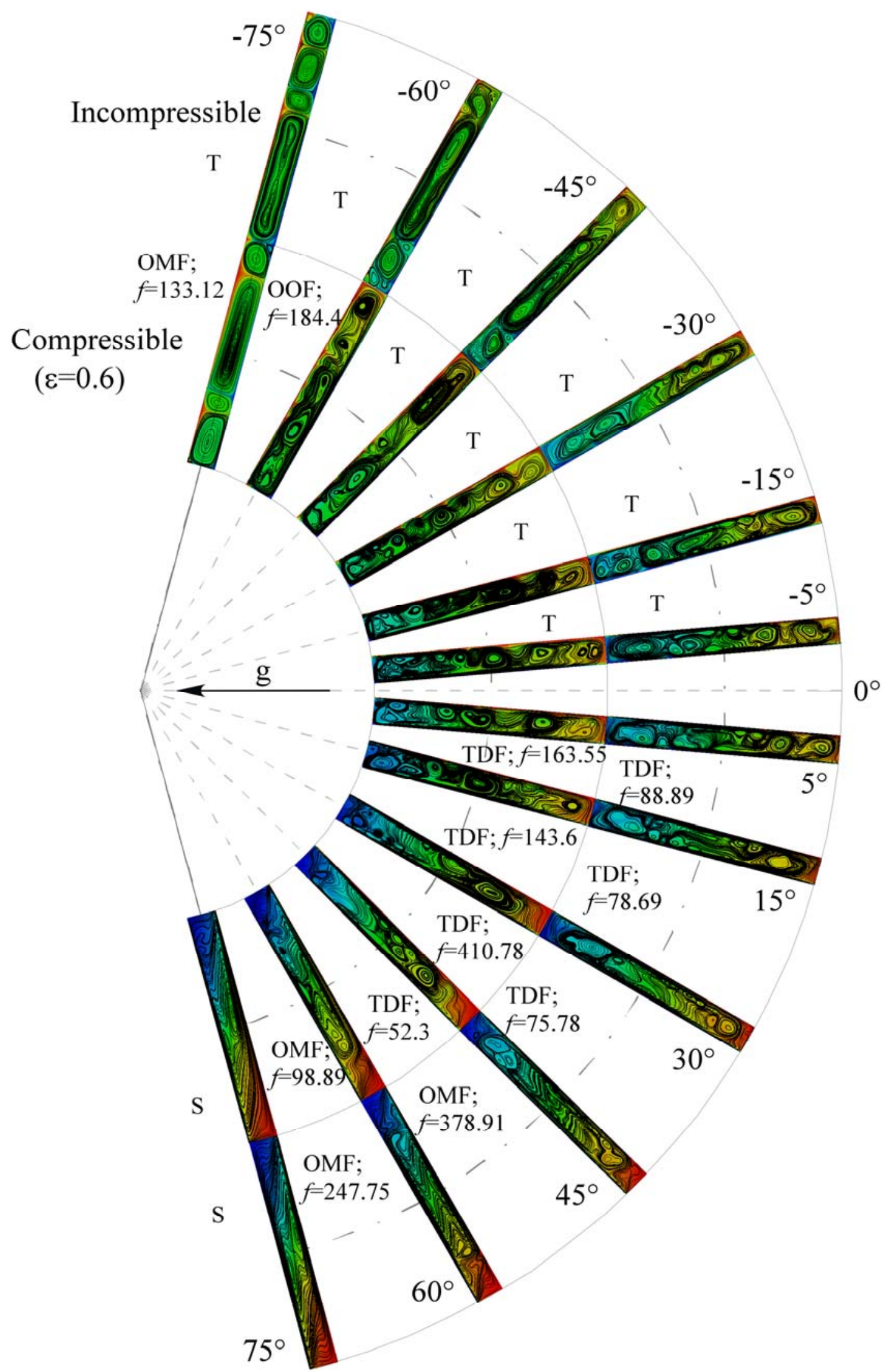

Figure 11: Map with different regimes for $\mathrm{Ra}=3 \times 10^{6}$. Legend: $\mathrm{S}-\mathrm{Steady} ; \mathrm{T}-\mathrm{Turbulent}$; OOF oscillatory flow with on frequency; OMF - oscillatory flow with more frequencies; TDF Turbulent flow with one dominant frequency. 
Individual and representative cases are discussed in detail in the following subsections. Understandably, we omit a description of the trivial condition corresponding to $\theta=90^{\circ}$ (in such a case quiescent and thermally diffusive conditions are established in the cavity). Following the same approach used in Sect. 4.1, the presentation of findings is articulated in two main parts or subsections corresponding to flows being obtained under the assumption of constant (Boussinesq model) or variable properties (NOB effects being properly captured).

\subsubsection{Boussinesq limit}

First we examine the cases with positive values of the angle $\theta$ for which the vertical component of buoyancy is expected to play a stabilizing role (vertical component of the imposed temperature gradients and gravity having opposite directions). Situations in which the inclination of the cavity leads to excitations of convective modes typical of the RB problem are then considered (vertical component of the imposed temperature gradients and gravity being concurrent, i.e. $\theta \leq 0$ ).

According to the results for $R a=1.9 \times 10^{5}$, when the inclination angle is varied from $0^{\circ}$ to $90^{\circ}$ the flow retains its steady state over the entire range of inclinations (as expected, given the stabilizing role of the heating-from-above effect). For $R a=3 \times 10^{5}$, however, the dynamics display a much more interesting and apparently counter-intuitive behaviour. Indeed, the initially oscillatory (periodic) flow found for the vertical cavity $\left(\theta=0^{\circ}\right)$, is taken over by a multi-frequency state as soon as the angle $\theta$ enters the interval of positive values (this can be seen for both $\theta=5^{\circ}$ and $\theta=15^{\circ}$ ). A further increase in $\theta$, however, causes the transition from the multi-modal state to steady flow, a condition that persists until $\theta$ attains its maximum value $\theta=90^{\circ}$ (corresponding to the system with heated horizontal top wall, i.e. the aforementioned quiescent degenerate state).

It can be seen that in all cases, the distinguishable circulations originally visible in the central region of the cavity tend to merge into one relatively large vortex located around its middle (back transition from a multi-roll state to a single roll state).

The dynamics for negative values of $\theta$ are more complicated. For $R a=1.9 \times 10^{5}$, any departure of $\theta$ from the two limiting situations corresponding to the boundary-layer regime $\left(\theta=0^{\circ}\right)$ or system uniformly heated from below $\left(\theta=-90^{\circ}, \mathrm{RB}\right.$ convection) in which convection is steady, causes the flow to become time-dependent. Time-dependence manifests itself via the continuous nucleation and merging of two circulations in the centre of the cavity as shown for $\theta=-15^{\circ}$ in Fig. 12. When the inclination angle is decreased towards $\theta=-75^{\circ}$, these features tend to be compressed in the very narrow space in the middle of the fluid domain.

For $R a=3 \times 10^{5}$, though the dynamics are still essentially oscillatory in the entire range of angles covering the interval $-90^{\circ}<\theta \leq 0^{\circ}$, the spatio-temporal behaviours displayed by the flow are considerably different with respect to those seen for $R a=1.9 \times 10^{5}$. For $\theta=-45^{\circ}$ as shown in Fig. 13, 
the two rolls initially created in the middle travel as time increases in opposite directions towards the ends of the cavity where they finally merge with the large circulations located there. The flow is anti-symmetric with respect to the mid-plane. Further increase in the inclination causes the narrowing of such vortices. For $\theta=-90^{\circ}$ (RB convection) stationary conditions are recovered with many steady rolls (multicellular state) being present in the cavity as shown in Fig. $4 \mathrm{~b}$.

The case $R a=3 \times 10^{6}$ deserves a separate discussion. The dynamics are rather turbulent. Interestingly, when the inclination is such that convective modes typical of RB convection are excited $(\theta<0)$, turbulence manifests essentially in the frequency spectrum (the patterning behaviour still giving rise to recognizable regular structures). On the other hand, if the temperature gradient has a vertical component corresponding to heating the system from above $(\theta>0)$, the complexity of the flow can be seen especially in terms of topology of the related streamlines (featured by many eddies, see, e.g. Fig. 14). However, for $\theta=45^{\circ}$, the basic structure of the flow is very similar to that obtained for $\theta=0$ and both $R a=1.9 \times 10^{5}$ and $R a=3 \times 10^{5}$. There are two smaller circulations located in the middle and two larger circulations located at each end of the cavity (Fig. 15).

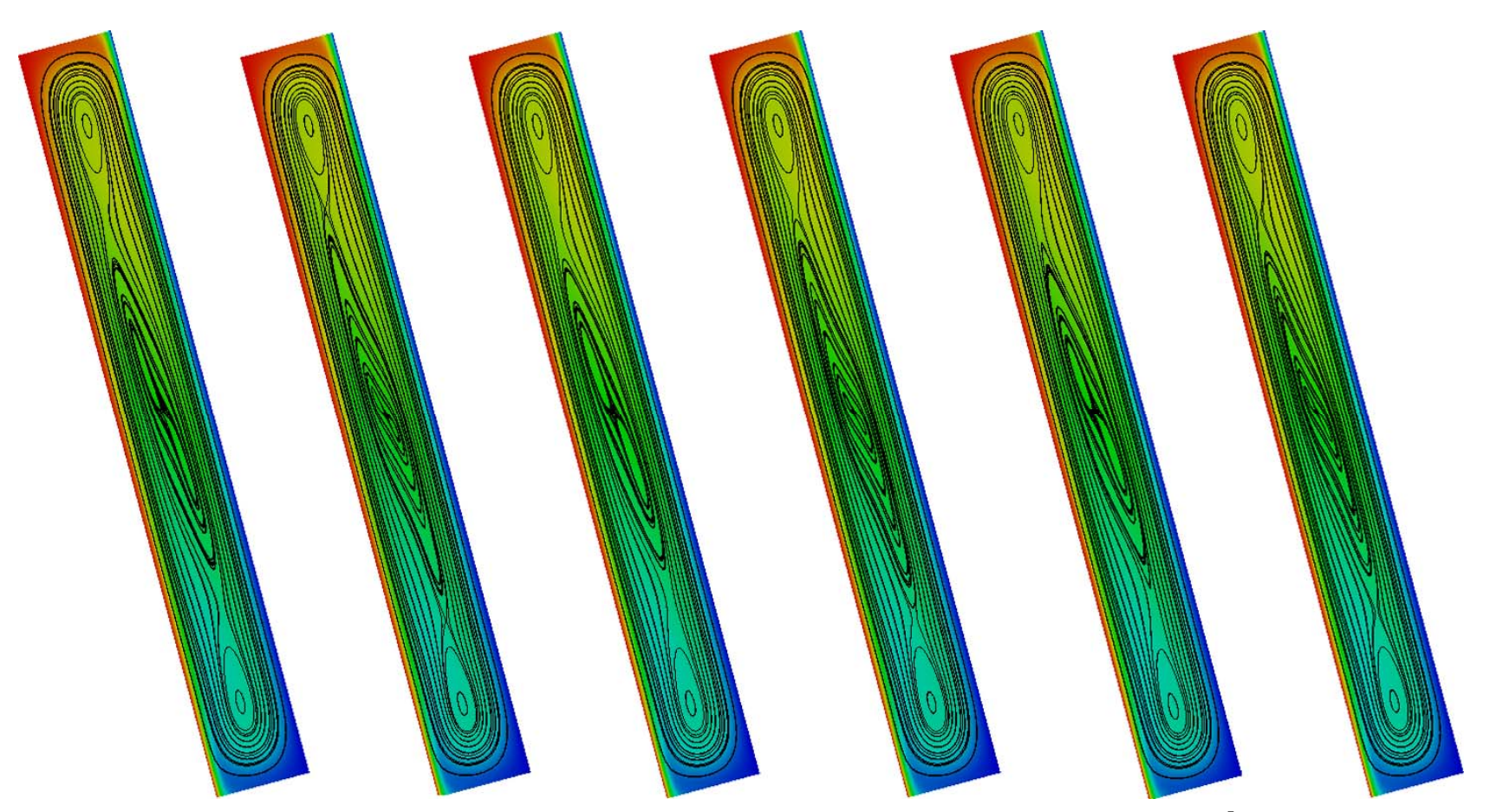

Fig. 12: Subsequent snapshots of convection (incompressible flow, $\mathrm{Ra}=1.9 \times 10^{5}, \theta=-15^{\circ}, f_{D}=$ 97.25). 


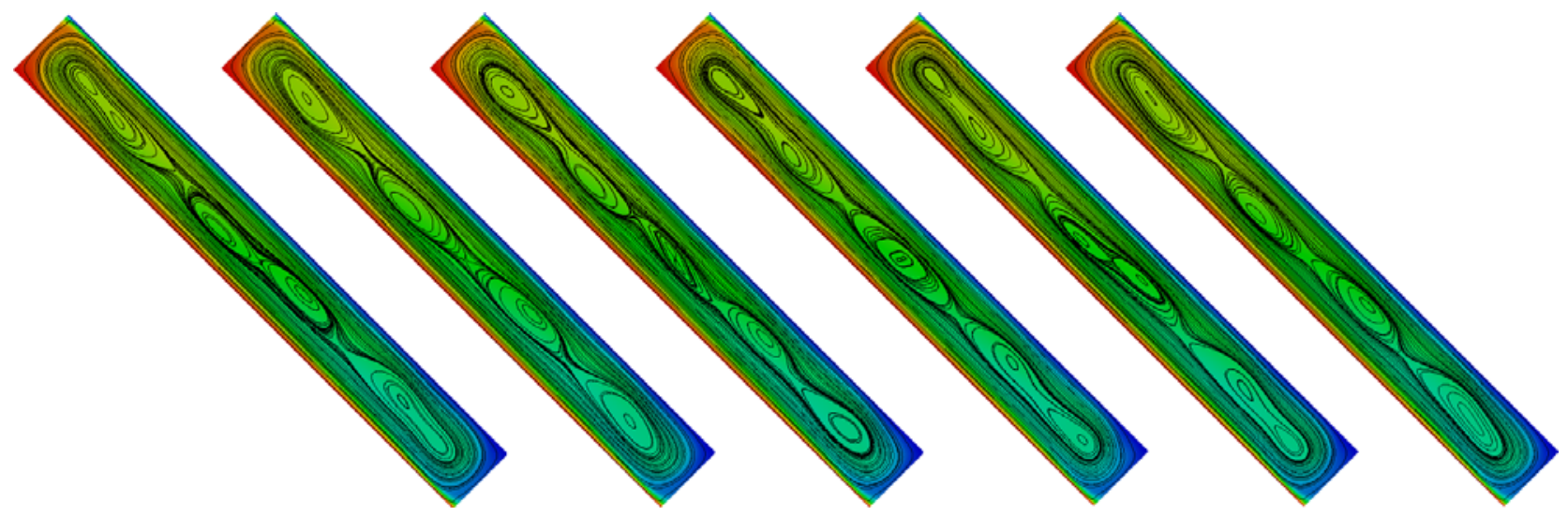

Fig. 13: Subsequent snapshots of convection (incompressible flow, $\mathrm{Ra}=3 \times 10^{5}, \theta=-45^{\circ}, f_{D}=$ 122.45).

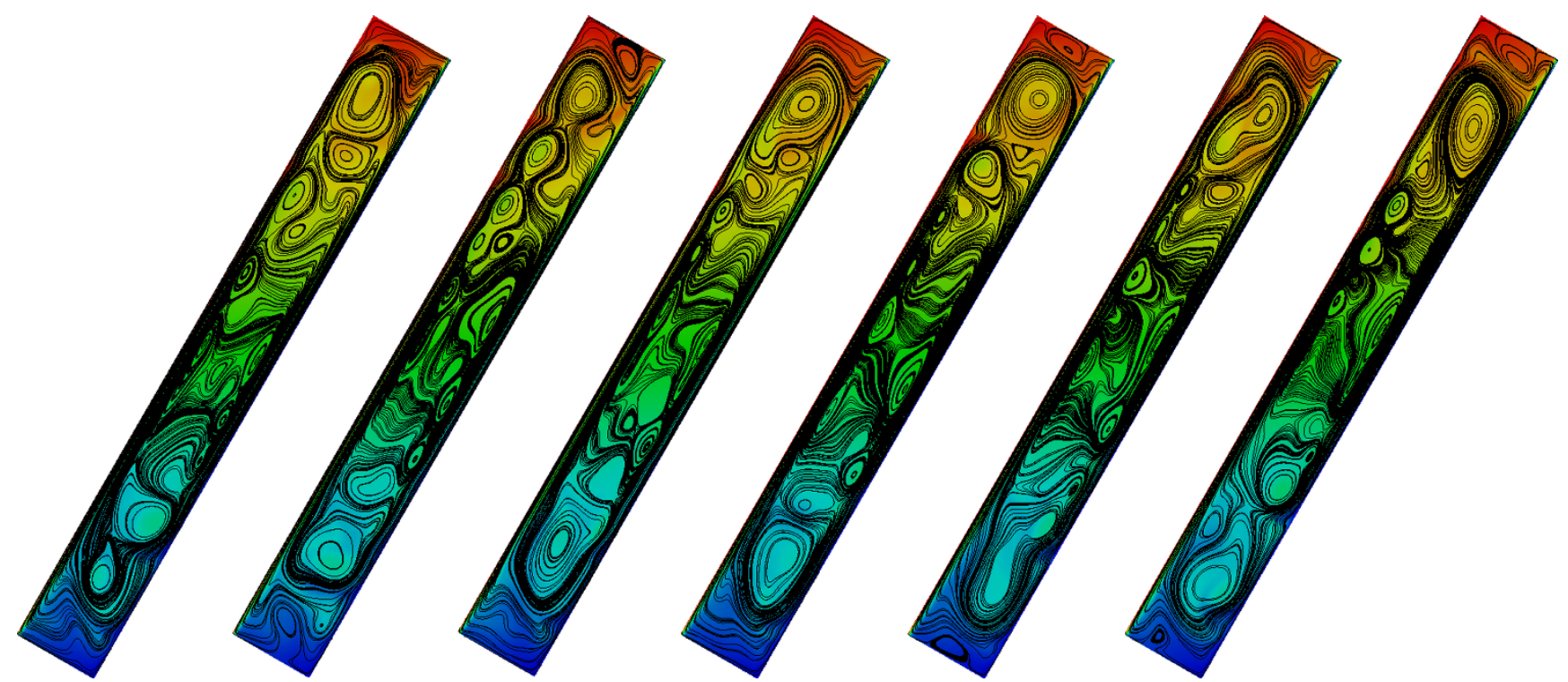

Fig. 14: Subsequent snapshots of convection (incompressible flow, $\mathrm{Ra}=3 \times 10^{6}, \theta=30^{\circ}, f_{D}=$ 75.78).

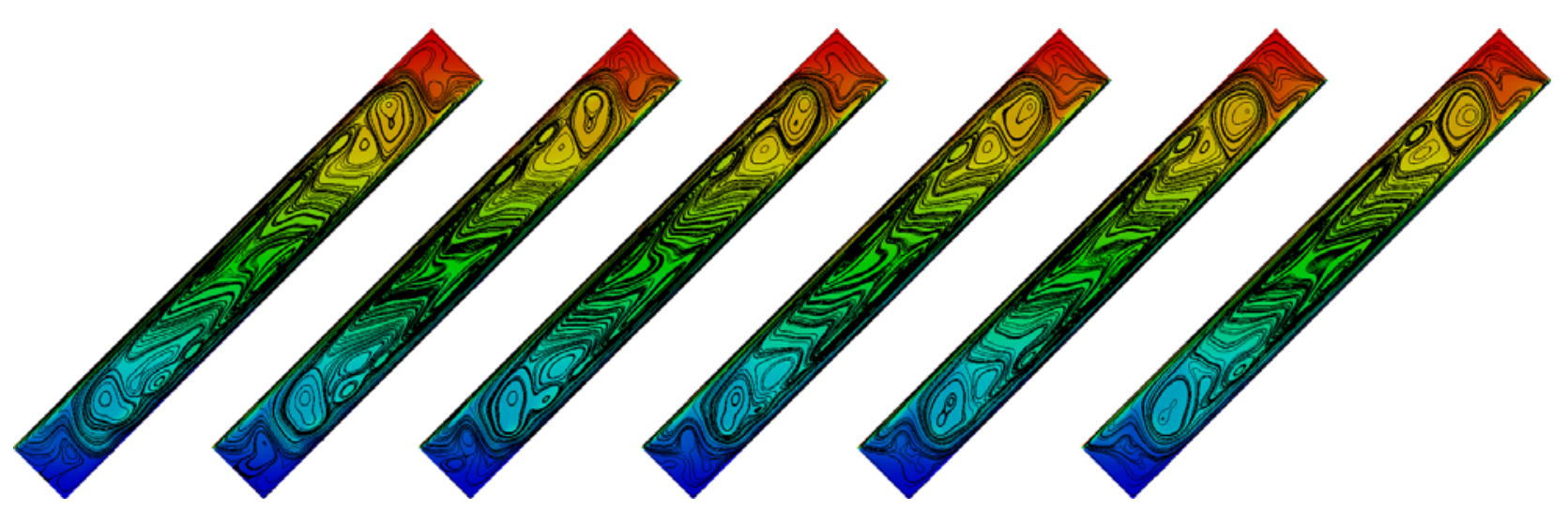

Fig. 15: Subsequent snapshots of convection (incompressible flow, $\mathrm{Ra}=3 \times 10^{6}, \theta=45^{\circ}, f_{D}=$ 378.91). 


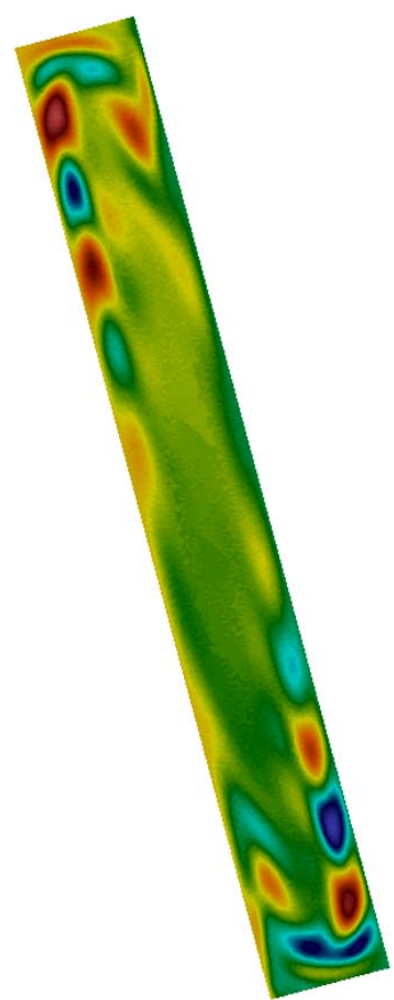

a) $-15^{\circ}$

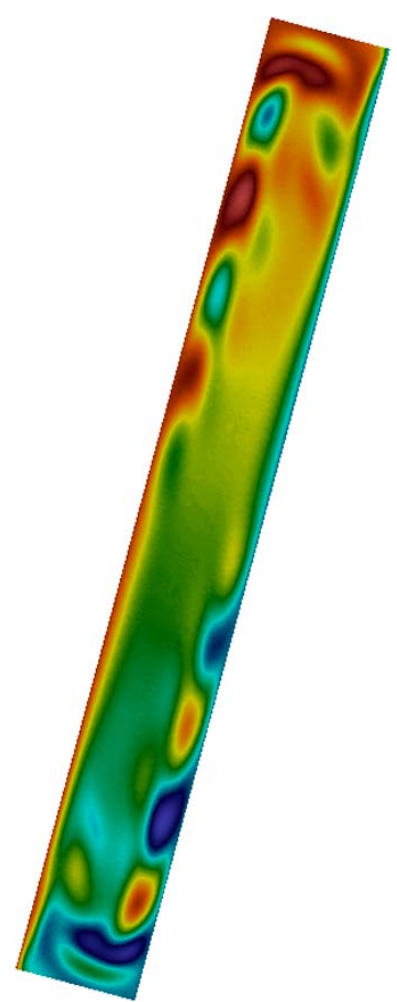

b) $15^{\circ}$

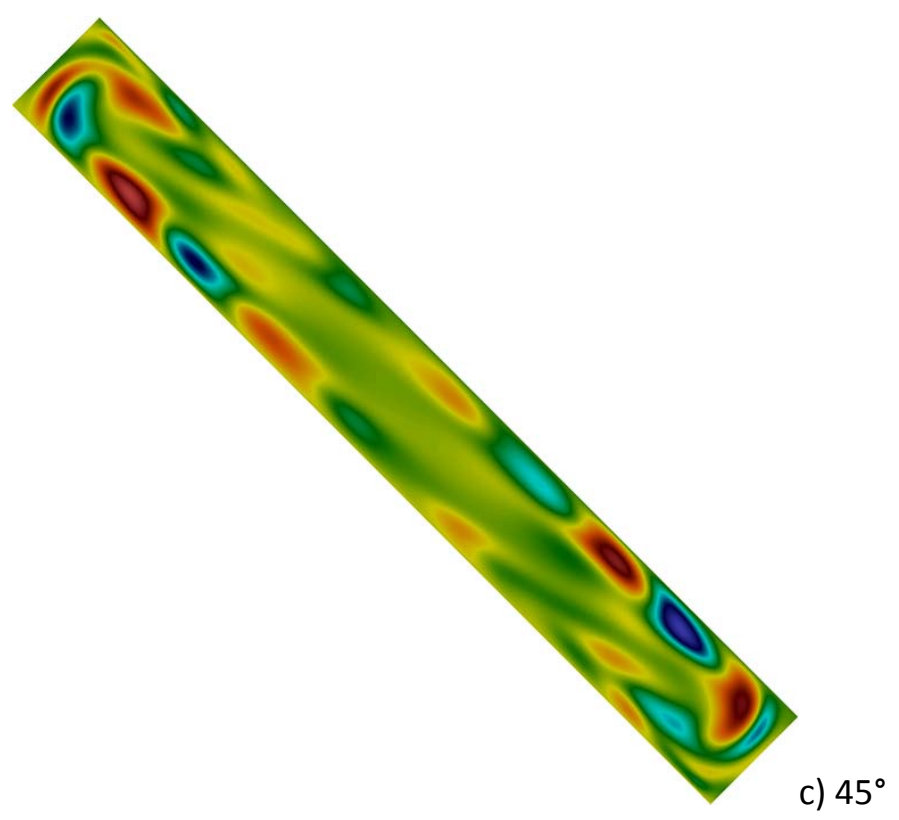

Fig. 16: Temperature disturbances for $\mathrm{Ra}=3 \times 10^{5}$ (incompressible flow).

Figure 16 reveals the typical structure of the temperature disturbances obtained subtracting the time-averaged field to the corresponding instantaneous field. It can be seen that time-dependence is essentially due to the presence of two counter-propagating waves travelling along the left and right walls. 
The two waves apparently originate from the middle of the cavity and satisfy the condition of skew symmetry already discussed for the velocity field.

\subsubsection{Variable properties}

Interestingly, when the variable-properties approach is used, the qualitative differences between the cases with $R a=1.9 \times 10^{5}$ and $R a=3 \times 10^{5}$ become much less evident.

By increasing the inclination angle beyond $15^{\circ}$ the flow becomes steady and only one vortex appears in the cavity for both Rayleigh numbers. For $\theta$ smaller than $-15^{\circ}$ two big circulations embedded in the general convective pattern can be distinguished. Such circulations merge around $\theta$ $=-75^{\circ}$ giving rise to a single elongated cell.

Leaving aside such qualitative aspects, however, the most interesting differences with respect to the incompressible case can be identified in terms of stability behaviour. Indeed, the impact of variableproperties on the spatio-temporal dynamics is not as trivial as one would imagine.

As an example, moving from the Boussinesq to the variable-properties approach makes the flow more stable when $\theta$ is in a given neighbourhood of $\theta=-90^{\circ}$ for $R a=1.9 \times 10^{5}$, and more unstable when $\theta$ is in a given neighbourhood $(\theta<0)$ of $\theta=0^{\circ}$ for $R a=3 \times 10^{5}$. The former trend must be ascribed to the increase in the Rayleigh number required for the onset of time-dependence in classical RB convection when NOB effects are taken into account (compare, e.g., the results by Goldhirsch et al. [75] and those by Bouabdallah et al. [76]). Vice versa, the latter is clearly due to the well-known shrinkage of the instability threshold (for the onset of oscillatory flow) induced by variable-property effects when the flow is in the boundary-layer regime (Le Quéré et al. [30]).

Figures 17-20 show some representative examples. The formation of localised circulations just below the middle of the cavity travelling in the positive $y$-direction and merging in the upper part of the cavity can be seen in all the figures for $R a=1.9 \times 10^{5}$ and $R a=3 \times 10^{5}$. However, the number of such rolls changes according to the specific set of parameters considered. For $R a=1.9 \times 10^{5}$ the number of rolls decreases from $\mathrm{m}=3$ for $\theta=15^{\circ}$ to $\mathrm{m}=2$ for $\theta=-15^{\circ}$ (Figs. 17 and 18). For $R a=$ $3 \times 10^{5}$ this number increases from $\mathrm{m}=3$ for $\theta=15^{\circ}$ to $\mathrm{m}=4$ for $\theta=-15^{\circ}$ (Figs. 19 and 20). 
Accepted for publication in the Int, J. Heat Mass Transfer on 19 December 2017

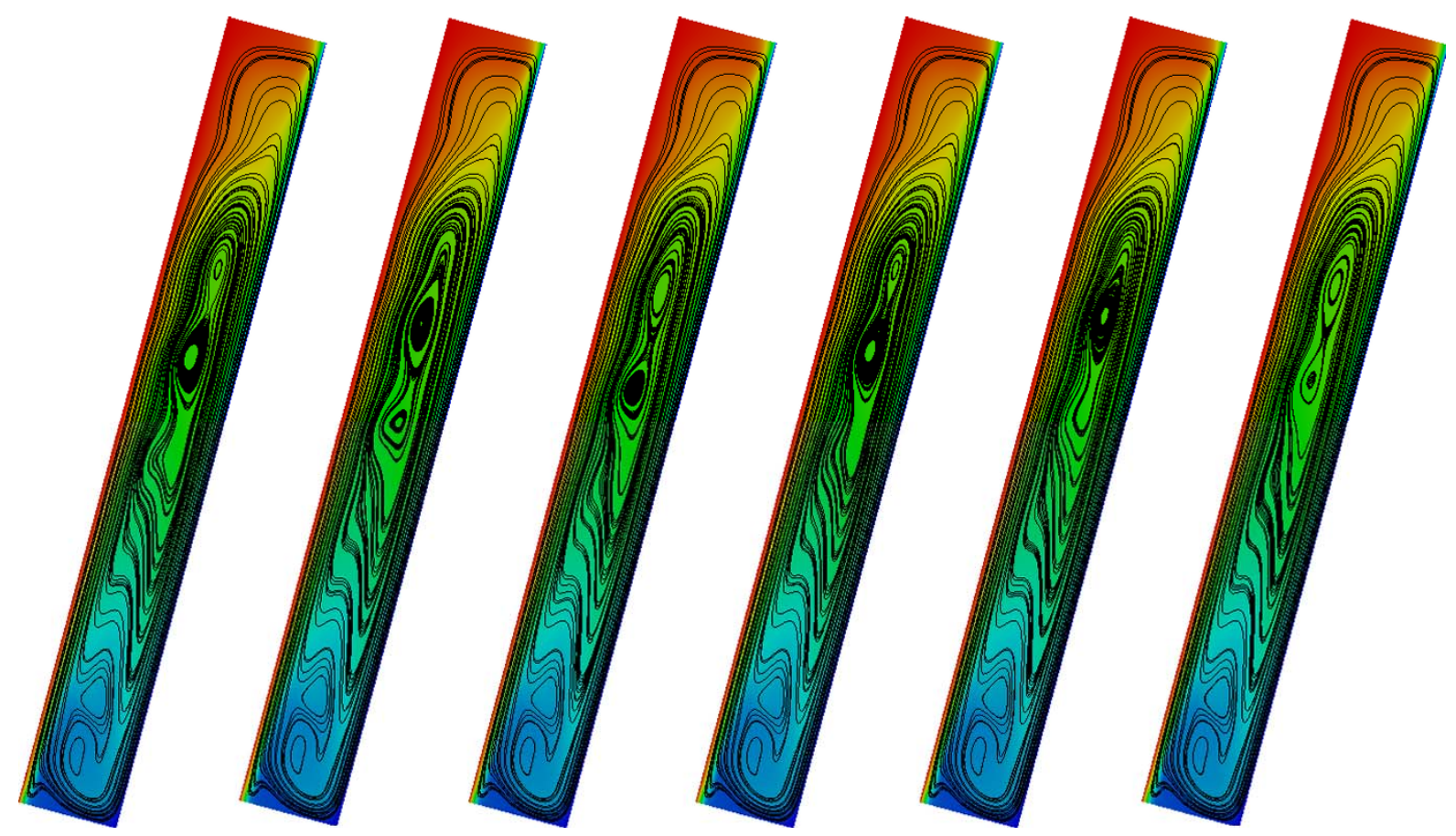

Fig. 17: Subsequent snapshots of convection (compressible flow, $\mathrm{Ra}=1.9 \times 10^{5}, \varepsilon=0.6, \theta=15^{\circ}, f_{D}=$ 127.06).

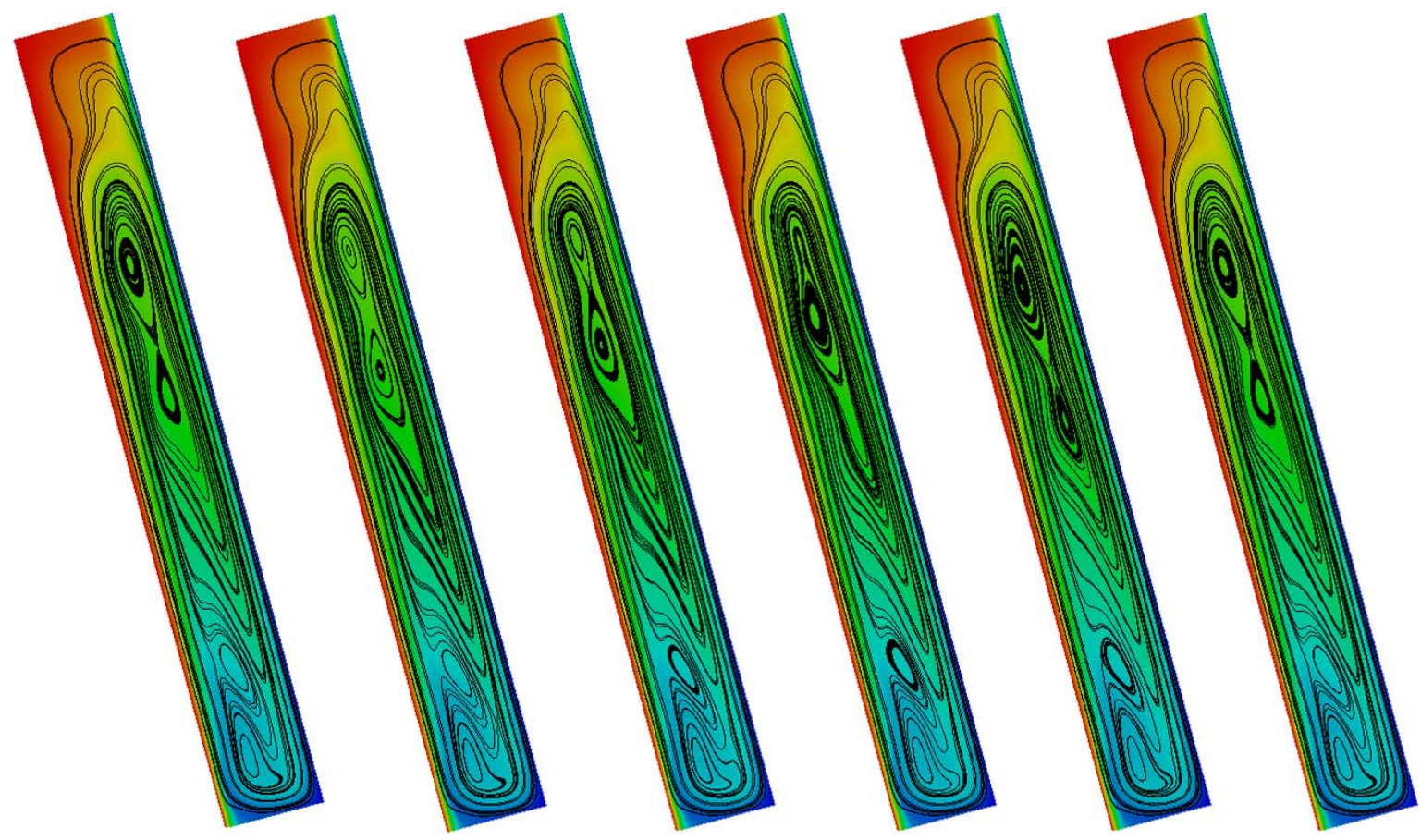

Fig. 18: Subsequent snapshots of convection (compressible flow, $\mathrm{Ra}=1.9 \times 10^{5}, \varepsilon=0.6, \theta=-15^{\circ}, f_{D}$ $=107.12$ ).

29 


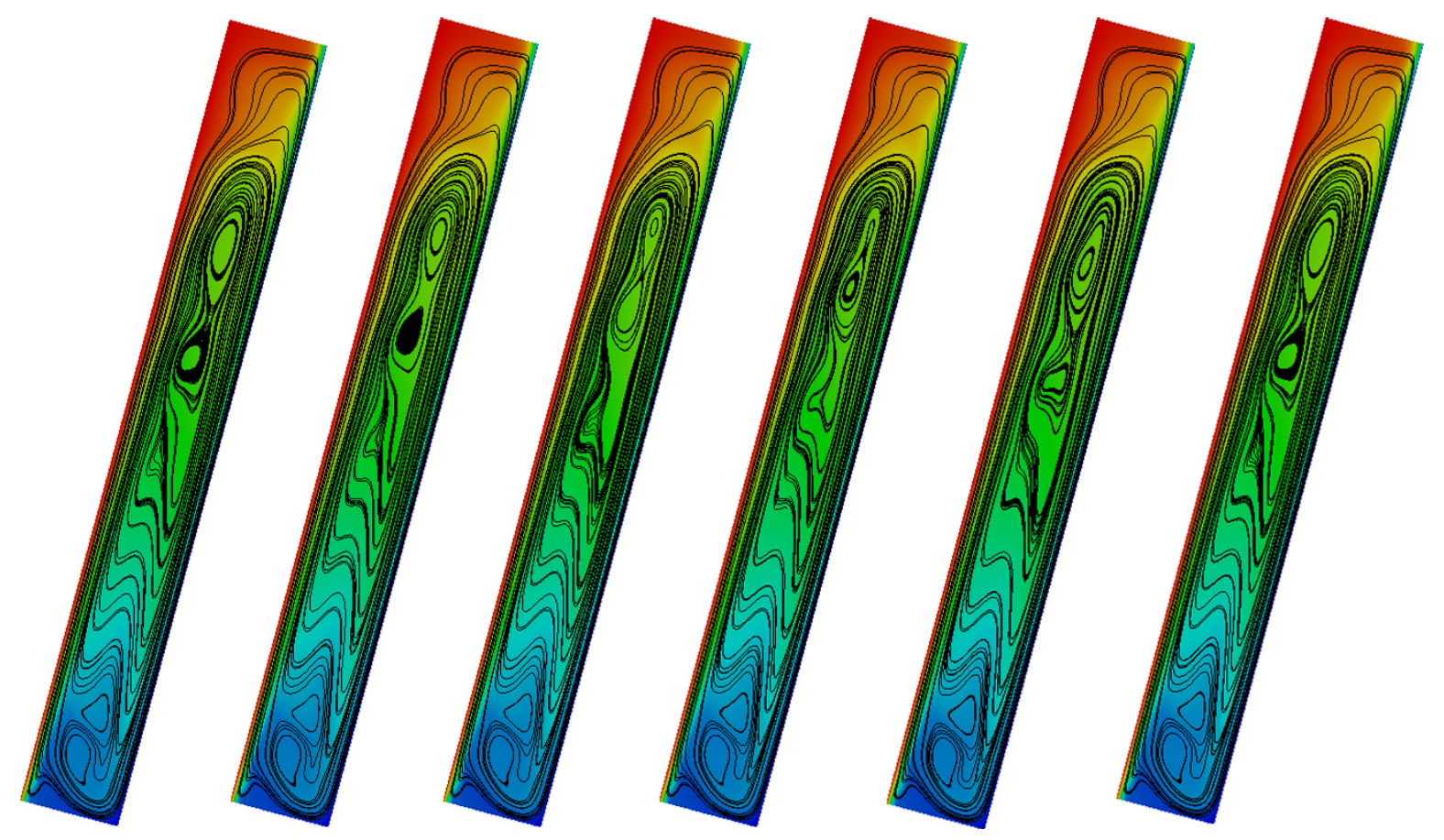

Fig. 19: Subsequent snapshots of convection (compressible flow, $\mathrm{Ra}=3 \times 10^{5}, \varepsilon=0.6, \theta=15^{\circ}, f_{D}=$ 146.13).

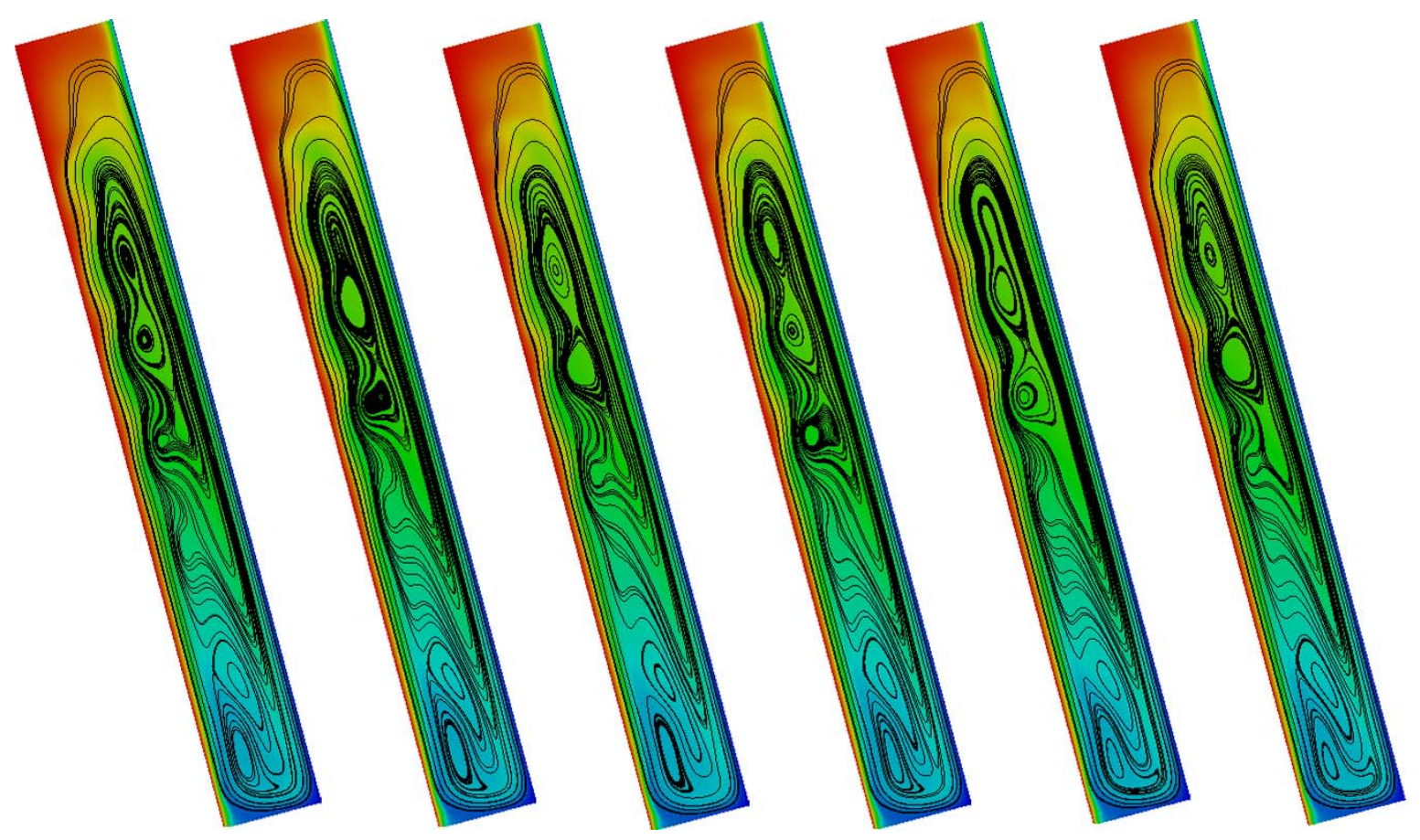

Fig. 20: Subsequent snapshots of convection (compressible flow, $\mathrm{Ra}=3 \times 10^{5}, \varepsilon=0.6, \theta=-15^{\circ}, f_{D}=$ 170.44).

Another notable distinguishing mark with respect to the incompressible case can be identified in the dominant role played now by the instability of the thermal boundary layer on the heated side. This can be clearly seen in Figs. 21 and 22, which show the instantaneous fluctuations of temperature. The disturbance nodes are located in the region near the hot wall and spread continuously from the 
bottom of the cavity towards its top. This is in line with the observed behaviour of the flow from a purely kinematic point of view (with circulations originating from the middle of the cavity and travelling upwards).

For the incompressible case, as discussed to a certain extent in Sect. 4.1.2, the patterning behaviour is quite different. The presence of a travelling wave in a given neighbourhood of the condition $\theta=0^{\circ}$ is not limited to the hot thermal boundary layer. As clearly shown in Fig. 16, we could identify trains of spatially spreading disturbances close to both hot and cold sides (where waves travel upward in the hot thermal boundary layer and in the opposite direction in the cold). Indeed, the interplay between these two counterpropagating waves was the main driver for the dynamics discussed in Sect. 4.2.1 (about circulations nucleating in the central area of the fluid domain and then giving rise to two different streams of rolls spreading from the centre towards the top and the bottom, respectively).

As noticed by Bouloumou et al. [32], a justification for such modifications can be found in the realisation that when the variable-properties approach is used, the heating of the flow along the hot wall becomes more efficient than the cooling along the cold wall. This results in a significant change in the relative thickness of the two side thermal boundary layers as revealed by Figs. 17-20. Figures 21 and 22 simply confirm that the symmetry rupture produced by the NOB effects is responsible for the suppression of one of such waves, which explains why in the compressible case the spatio-temporal mechanism with circulations being continuously produced in the centre is taken over by a one-way disturbance transport process.

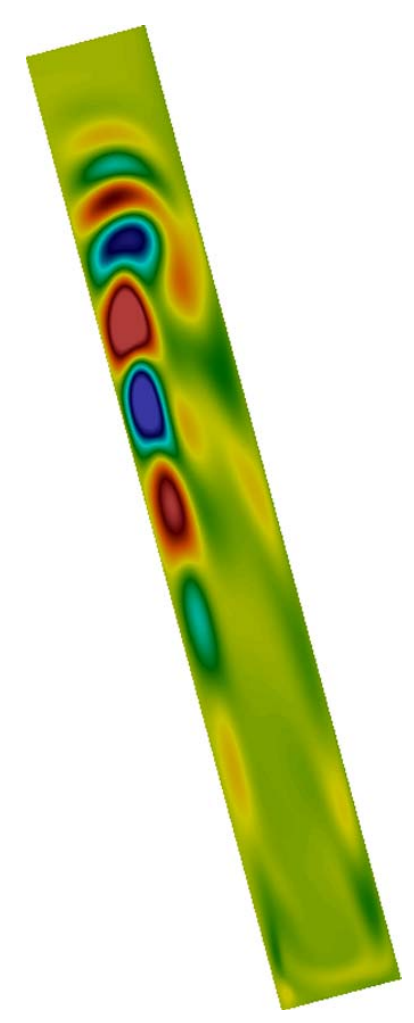

a) $-15^{\circ}$

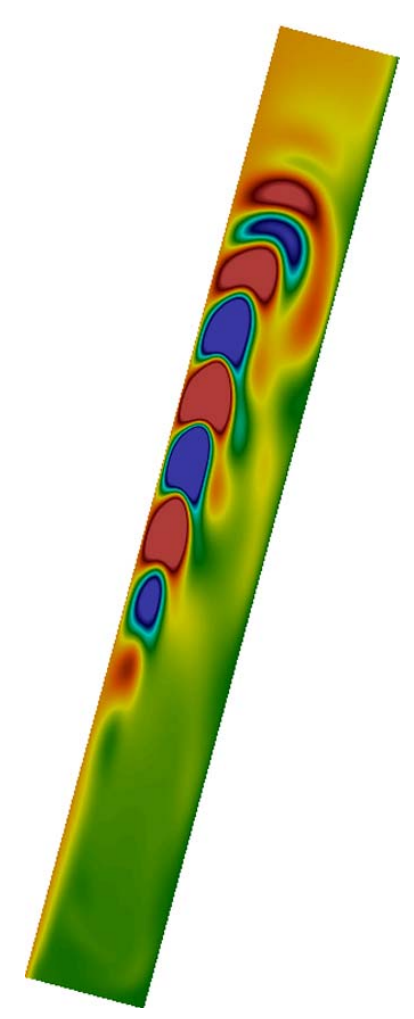

b) $15^{\circ}$

Fig. 21: Instantaneous temperature field for $\mathrm{Ra}=1.9 \times 10^{5}$ (compressible flow, $\varepsilon=0.6$ ). 


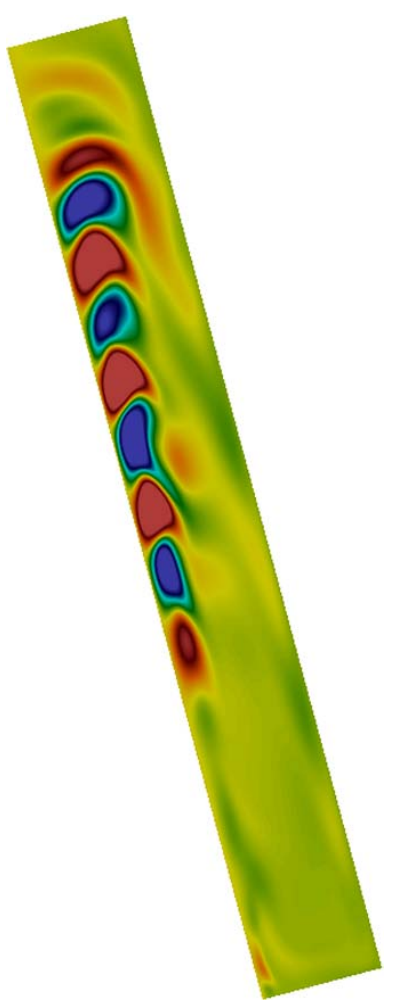

a) $-15^{\circ}$

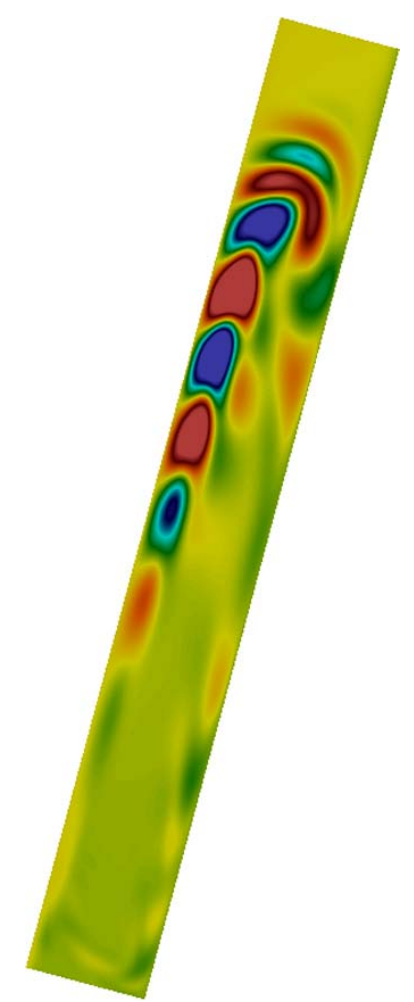

b) $15^{\circ}$

Fig. 22: Instantaneous temperature field for $\mathrm{Ra}=3 \times 10^{5}$ (compressible flow, $\varepsilon=0.6$ ).

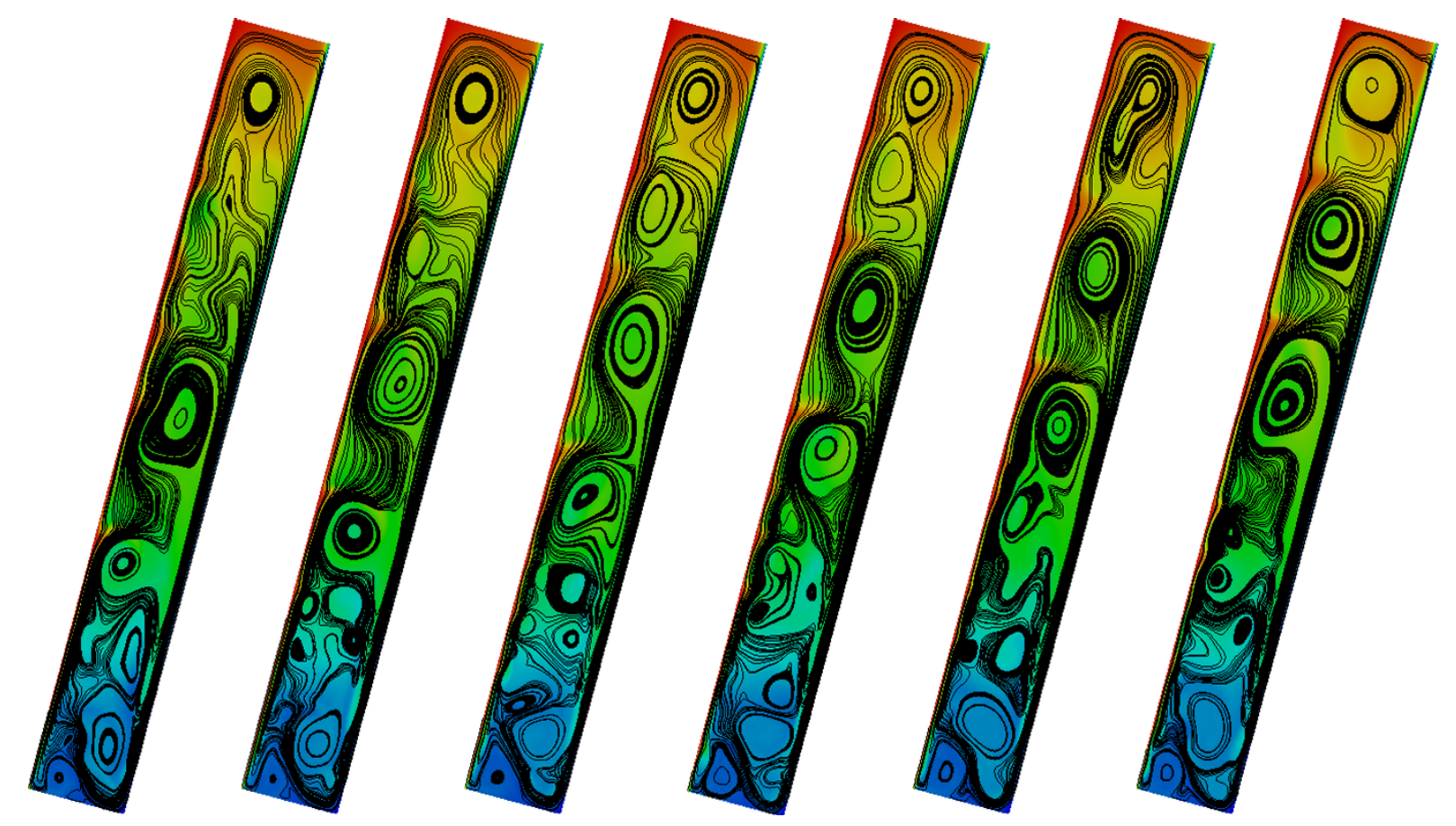

Fig. 23: Subsequent snapshots of convection (compressible flow, $\mathrm{Ra}=3 \times 10^{6}, \varepsilon=0.6, \theta=15^{\circ}, f_{D}=$ 143.58). 


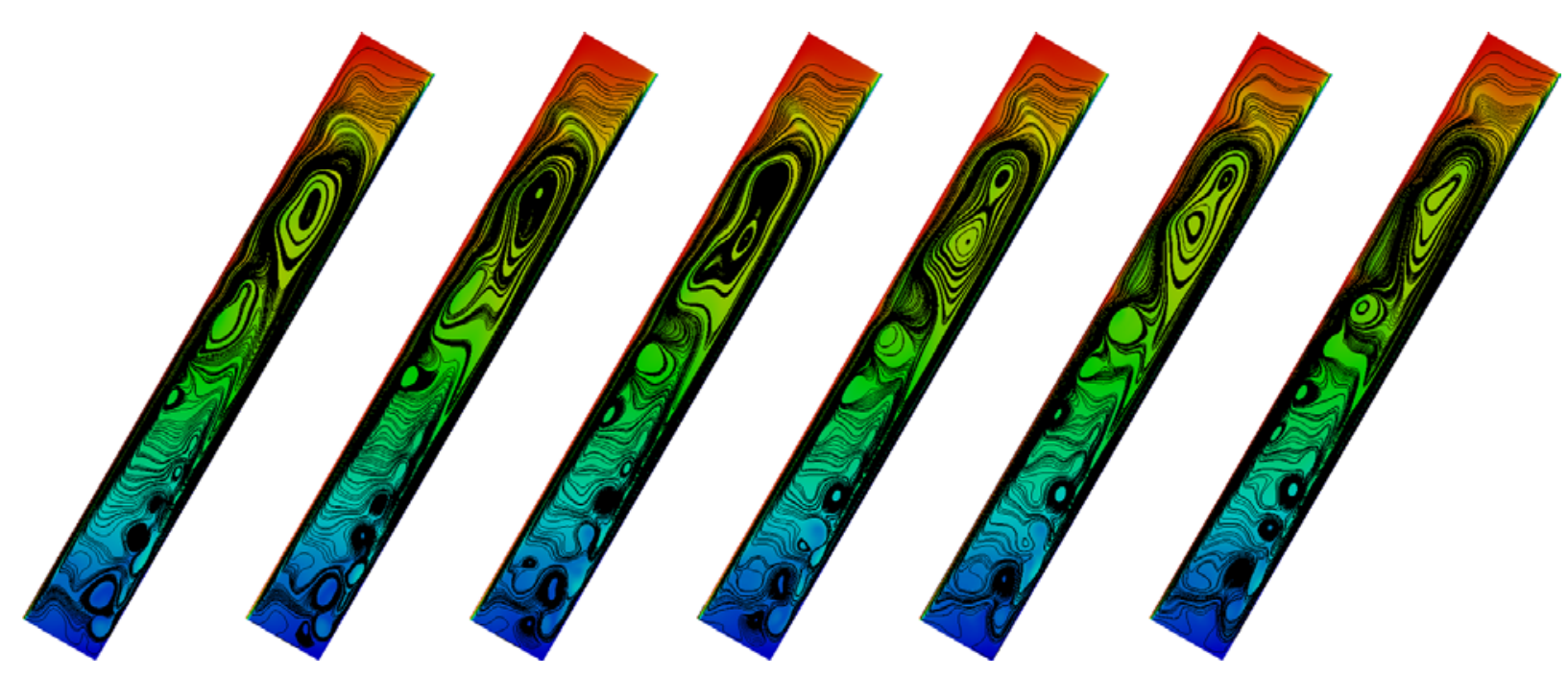

Fig. 24: Subsequent snapshots of convection (compressible flow, $\mathrm{Ra}=3 \times 10^{6}, \varepsilon=0.6, \theta=30^{\circ}, f_{D}=$ 410.78).

For $R a=3 \times 10^{6}$ the flow is generally chaotic. Multiple eddies seem to nucleate randomly in the cavity at different spatial locations as shown in Fig. 23 and 24. Nevertheless, some similarities with the lower Rayleigh-number cases can be observed. Travelling circulations are constantly being created, which then coalesce in the upper parts of the cavity. A peculiar scenario can be seen for $\theta=30^{\circ}$. A relatively extended stagnation region is formed in the upper part of the cavity, which resembles that typically produced for smaller values of Ra. For this inclination angle, moreover, the formation of small eddies seems to occur with a similar rate along both the hot and cold walls of the cavity.

\subsubsection{Frequency analysis}

It is possible to get additional useful insights into the dynamics described in the preceding sections through a critical analysis of the related frequency spectra. Such information is discussed in the present section with the aid of dedicated plots (Figs. 25-35). Such diagrams naturally complement Figs. 9-11 (they may be regarded as the natural counterpart of those figures); while the emphasis in Figs. 9-11 was on patterning behaviour, Figs. 25-35 provide specific information in terms of related spectral content of the flow.

In particular, we elaborate some general conclusions by filtering out specific details and concentrating on general trends.

Along these lines, by inspection of such plots (related to a numerical probe placed in the centre of the cavity), the reader will immediately realize that, in general, the flow is much more unstable when variable-density and variable-viscosity effects are taken into account (earlier transition to oscillatory flow and more complex frequency spectra). 


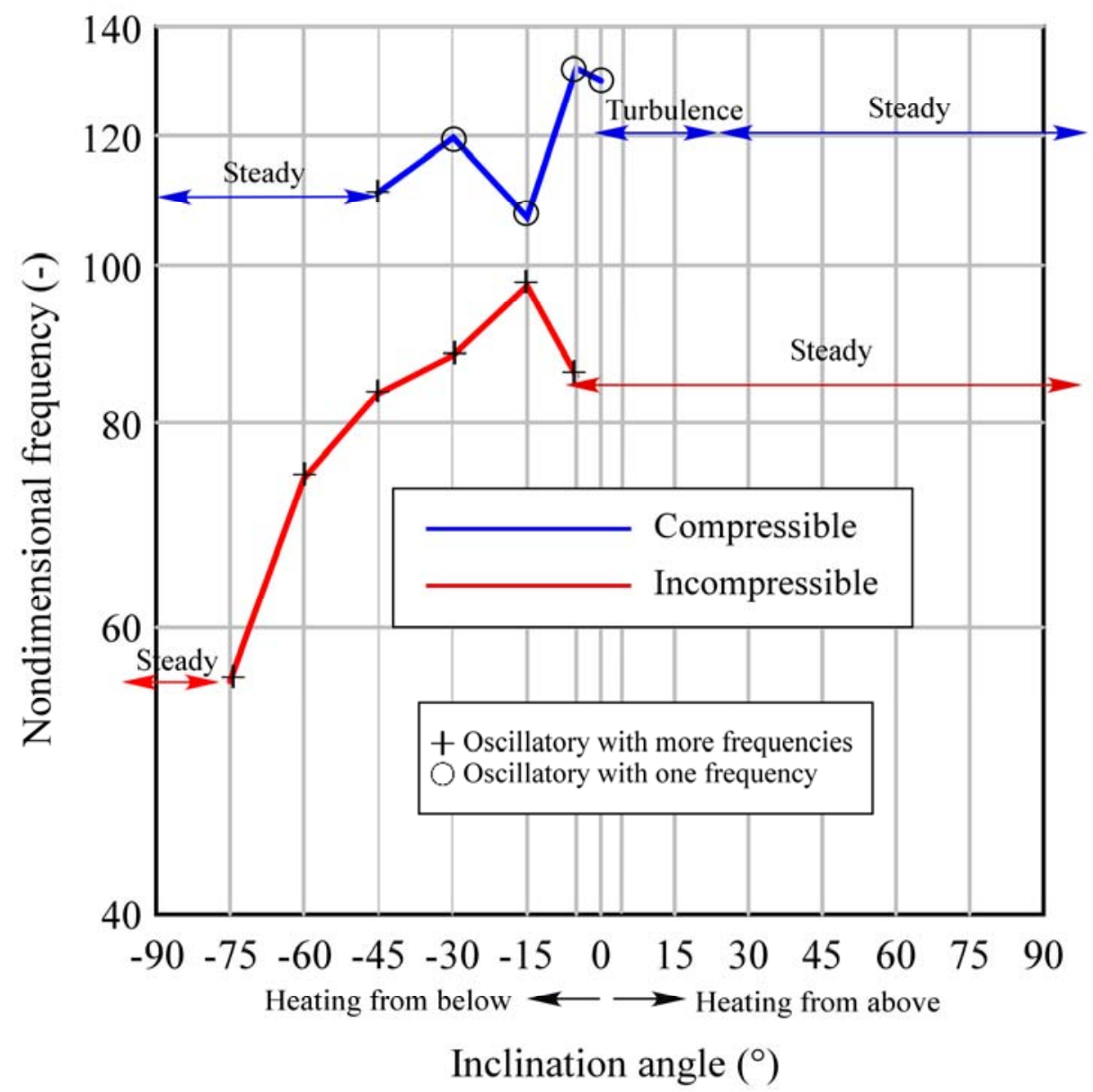

Fig. 25: Frequency analysis for $\mathrm{Ra}=1.9 \times 10^{5}$ : comparison between incompressible and compressible $(\varepsilon=0.6)$ flow solutions.

An initial indication along these lines can be gathered from an analysis of the plots showing the frequency (or frequency with maximum amplitude in the case of multi-modal or more complex spectra) as a function of the inclination angle for $\mathrm{Ra}=1.9 \times 10^{5}$ and $\mathrm{Ra}=3 \times 10^{5}$ (Figs. 25 and 29 , respectively).

For such values of the Rayleigh number, the flow is essentially steady for positive values of the angle $\theta$ regardless of whether NOB effects are at play or not (in qualitative agreement with the earlier experimental results by Saury et al. [35] for a cavity with $A=4$ ). Moreover, in agreement with [35], for negative values of $\theta$, starting from steady conditions for $\theta=-90^{\circ}$ (RB convection) the main frequency displays an increasing trend when the inclination becomes smaller (the absolute value of $\theta$ tends to zero). Though the qualitative trend is the same, when NOB effects are taken into account, the frequency corresponding to the most intense oscillation (maximum amplitude) undergoes a significant increase in the range $-45^{\circ} \leq \theta \leq-15^{\circ}$. 


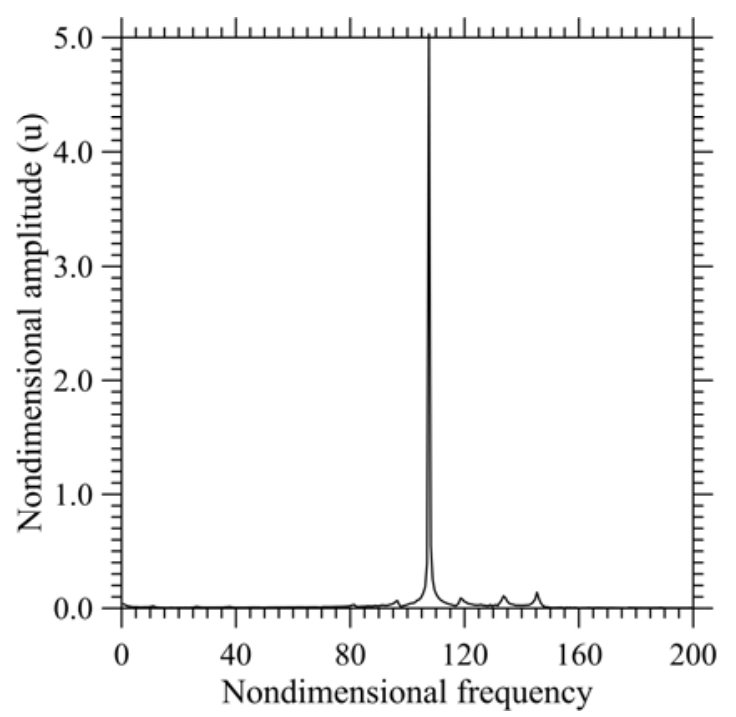

a) $-15^{\circ}$; $\operatorname{NOB}(\varepsilon=0.6)$

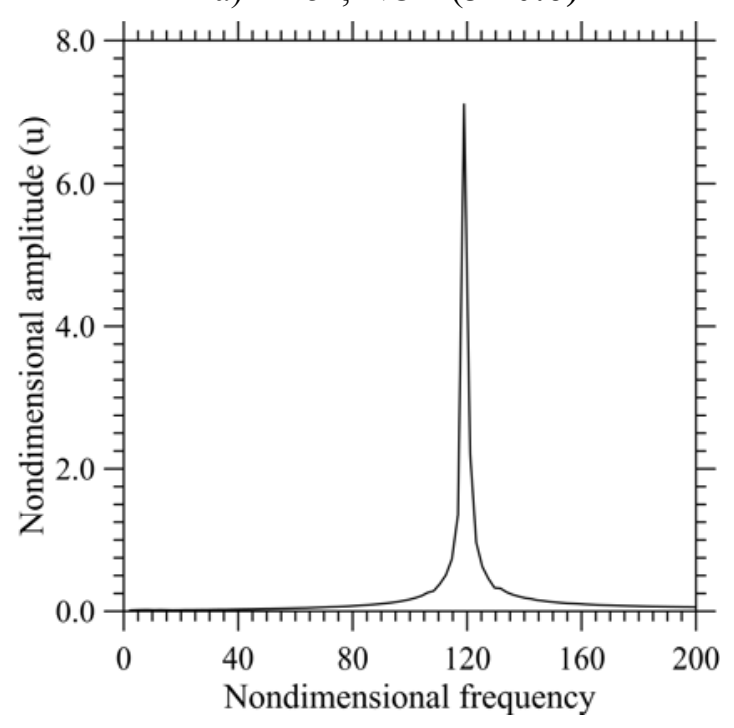

c) $-30^{\circ} ; \mathrm{NOB}(\varepsilon=0.6)$

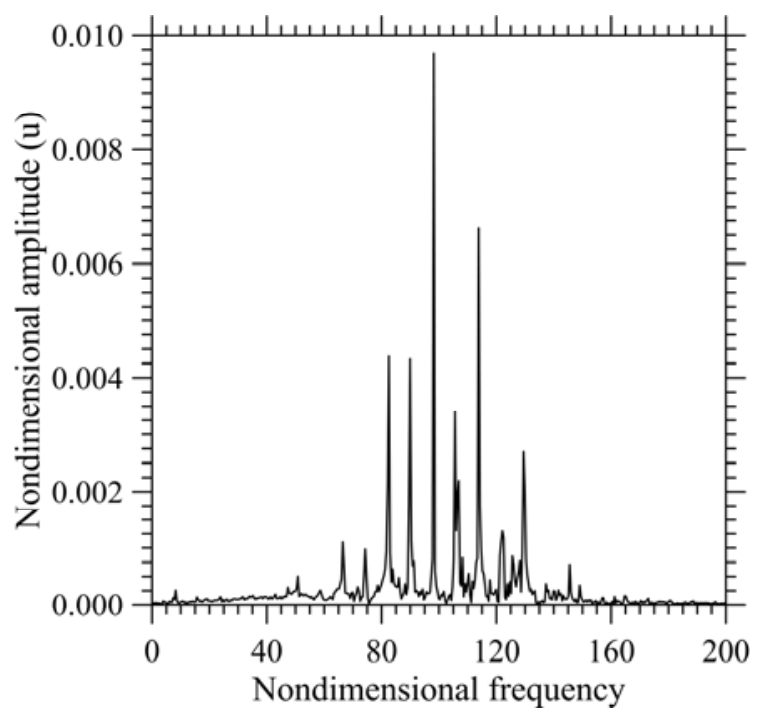

b) $-15^{\circ}$; Boussinesq

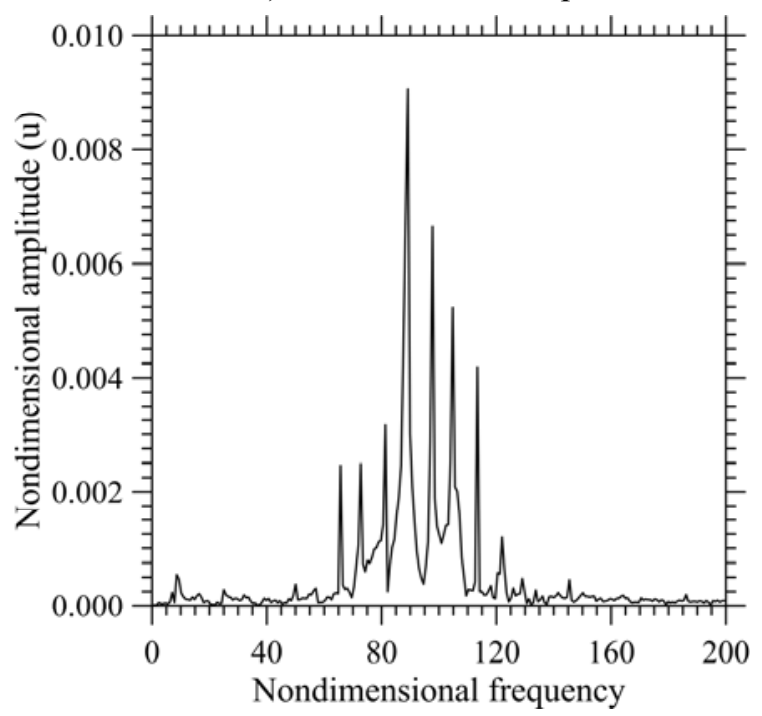

d) $-30^{\circ}$; Boussinesq

Fig. 26: Examples of frequency spectra for $\mathrm{Ra}=1.9 \times 10^{5}$.

Interestingly, for $\mathrm{Ra}=1.9 \times 10^{5}$ and $\theta<0$, though the frequency with maximum amplitude increases when NOB effects enter the dynamics, an opposite trend can be distinguished for what concerns the complexity of the frequency spectrum. Indeed, while for the cases with variable properties, purely periodic convection is obtained, multiple frequencies are present simultaneously in the spectrum when the Boussinesq model is used (Fig. 26), which is in qualitative agreement with earlier findings obtained by Williamson et al. [37] for the square cavity in conditions where this approximation is applicable. In order to discern the different cause-and-effect mechanisms responsible for such changes in the spectrum, we performed some additional simulations (for $\theta=-30^{\circ}$ ) allowing the imposed temperature difference to take intermediate values with respect to the two extremes indicated in Table II. The main outcomes of such simulations in terms of frequency spectra are summarised in Fig. 27 for $\varepsilon=0.1$ and $\varepsilon=0.2$, respectively. Additional useful information along these 
lines also follow naturally from Fig. 28, which shows the results of simulations carried out yet for $\varepsilon=0.6$, neglecting in one case the dependence of viscosity on temperature (intermediate model with variable density and constant viscosity, Fig. 28a) and fluid compressibility in the other case (constant density, variable viscosity, Fig. 28b)
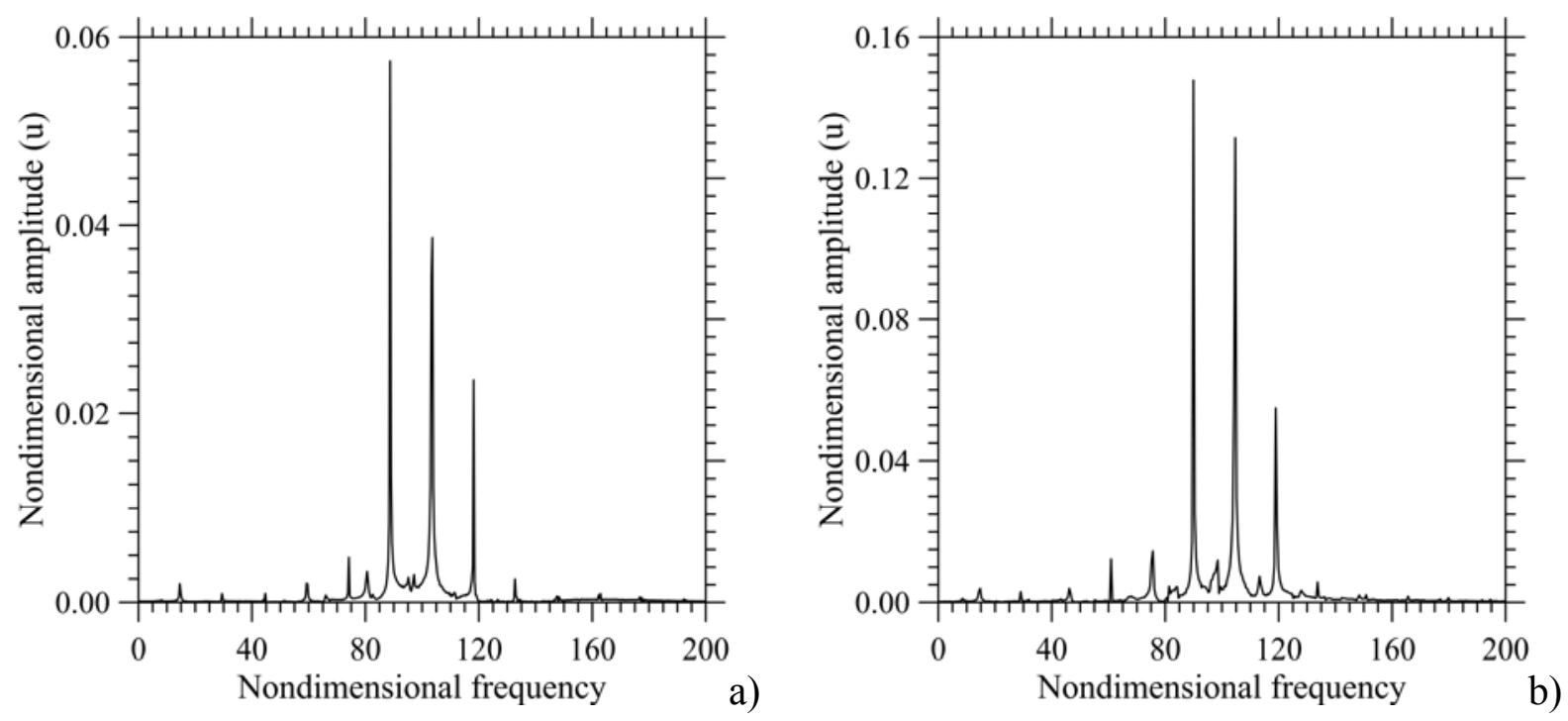

Fig. 27: Frequency spectra for $\mathrm{Ra}=1.9 \times 10^{5}, \theta=-30^{\circ}$ and different values of the temperature difference (variable properties, i.e. all NOB effects included): a) $\varepsilon=0.1$; b) $\varepsilon=0.2$.
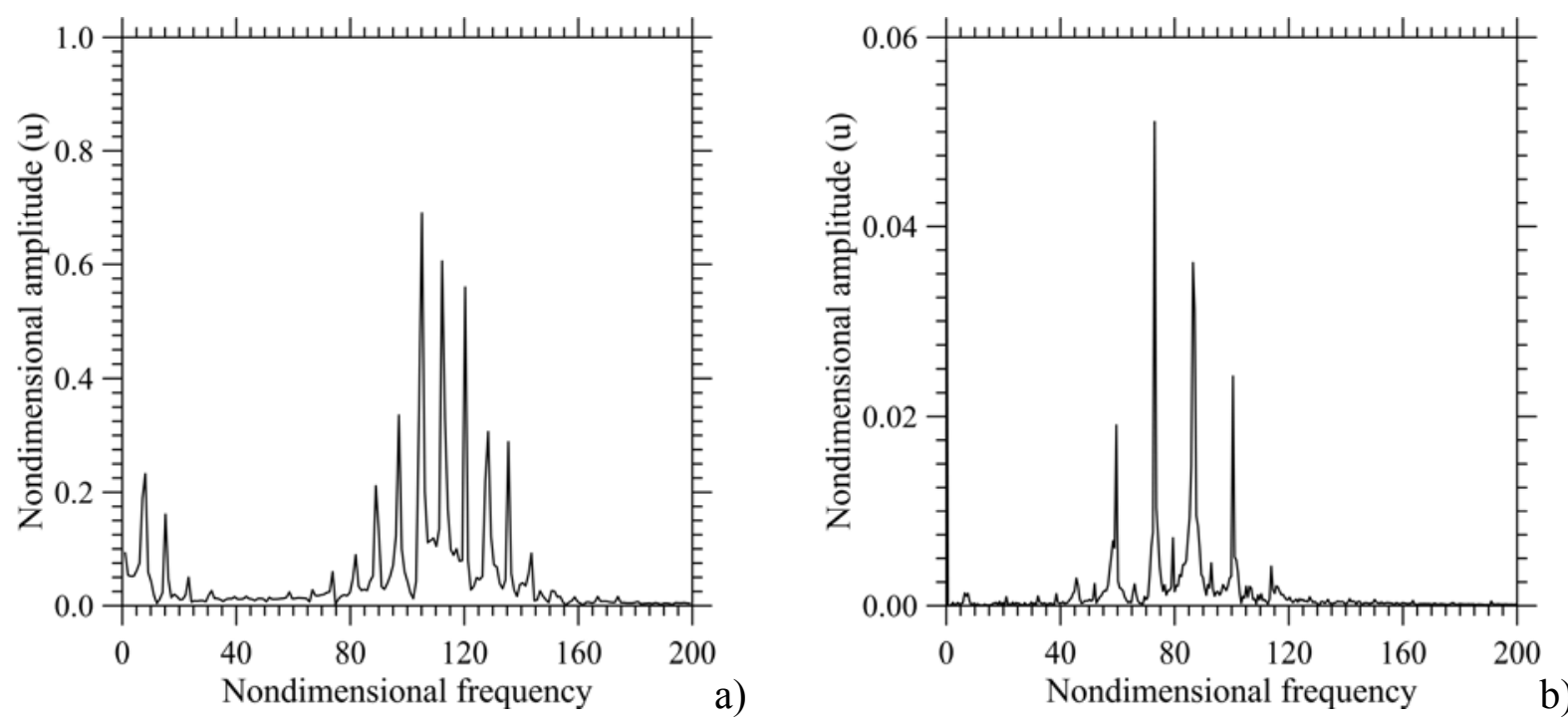

Fig. 28: Frequency spectra for $\mathrm{Ra}=1.9 \times 10^{5}, \varepsilon=0.6, \theta=-30^{\circ}$ and different models: a) Constant viscosity, variable density; b) Constant density, variable viscosity.

Comparison of Figs. 27a and $27 \mathrm{~b}$ leads to the interesting realisation that an increase in $\varepsilon$ from 0.1 to 0.2 (i.e. an increase in the applied temperature difference for a fixed value of the Rayleigh number), makes the amplitude of oscillations slightly higher. Such limited increments in $\varepsilon$, however, do not seem to modify significantly the main frequency (frequency with maximum amplitude), which remains more or less constant $\left(f_{\mathrm{D}} \cong 90\right)$ and almost equal to the corresponding value predicted by the 
numerical model with the Boussinesq approximation (with all properties assumed to be constant, Fig. 26d).

Figure $28 \mathrm{~b}$ provides some additional insights along these lines revealing that, for $\varepsilon=0.6$, if the density is kept constant while allowing the viscosity to vary, the amplitude of oscillations and the related frequency spectrum are very similar to that obtained with the Boussinesq approximation or with the variable-property model for $\varepsilon=0.1$. Vice versa, Fig. 28 a (yet for $\varepsilon=0.6$ and with the changes of viscosity neglected) finally demonstrates that compressibility can have a significant effect on both the amplitude and the main frequency (with the latter increasing from the aforementioned value $f_{\mathrm{D}} \cong 90$ to $f_{\mathrm{D}} \cong 120$, which is the same value visible in Fig. 26c).

Taken together all these figures indicate that the significant variations experienced by the frequency spectrum in Figs. 26c and 26d are essentially a consequence of the fluid compressibility (with the influence of variable viscosity being limited to producing a slight increase in the oscillation amplitude as $\varepsilon$ is increased). Comparison of Figs. 28a, 28b and 26c also supports the interesting conclusion that the increase in the oscillation amplitude is much higher when variable density and variable viscosity are considered at the same time.

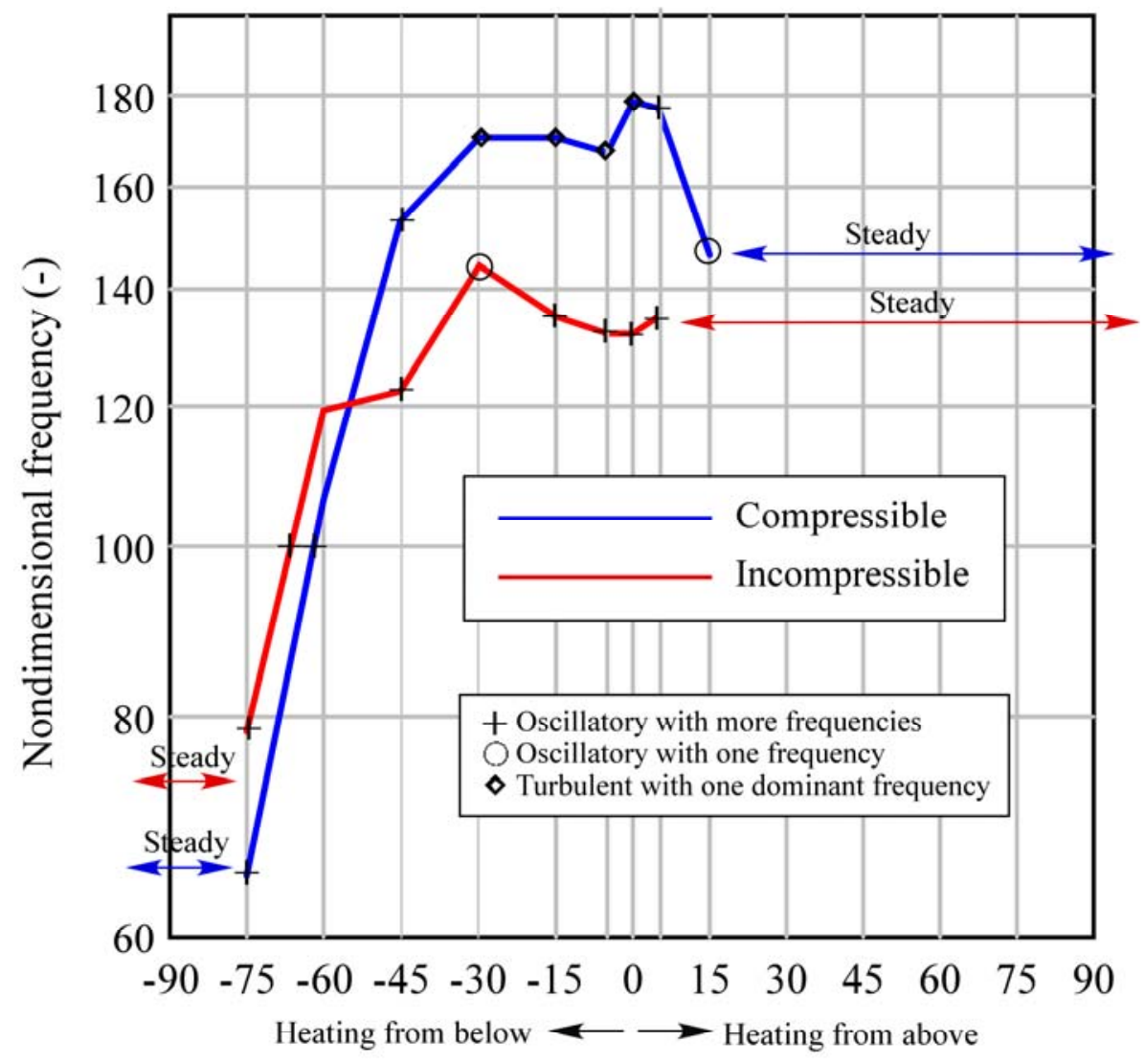

Inclination angle $\left({ }^{\circ}\right)$

Fig. 29: Frequency analysis for $\mathrm{Ra}=3 \times 10^{5}$ : comparison between incompressible and compressible ( $\varepsilon=0.6)$ flow solutions. 


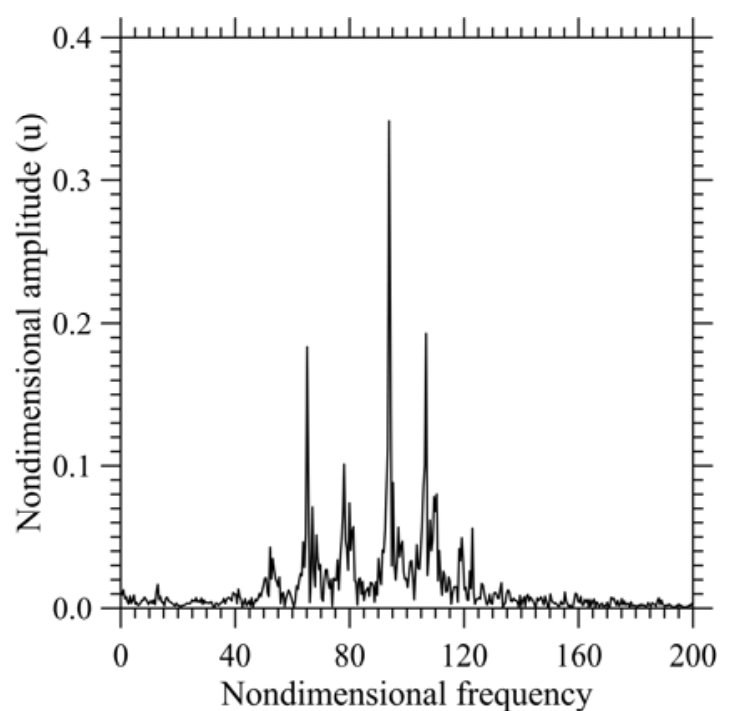

a) $-75^{\circ}$; $\mathrm{NOB}(\varepsilon=0.6)$

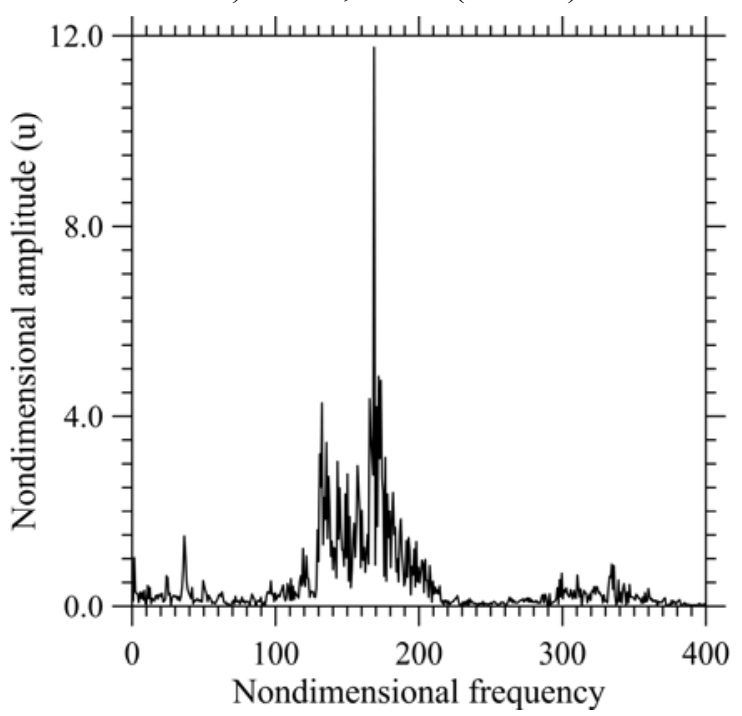

c) $-5^{\circ}$; $\mathrm{NOB}(\varepsilon=0.6)$

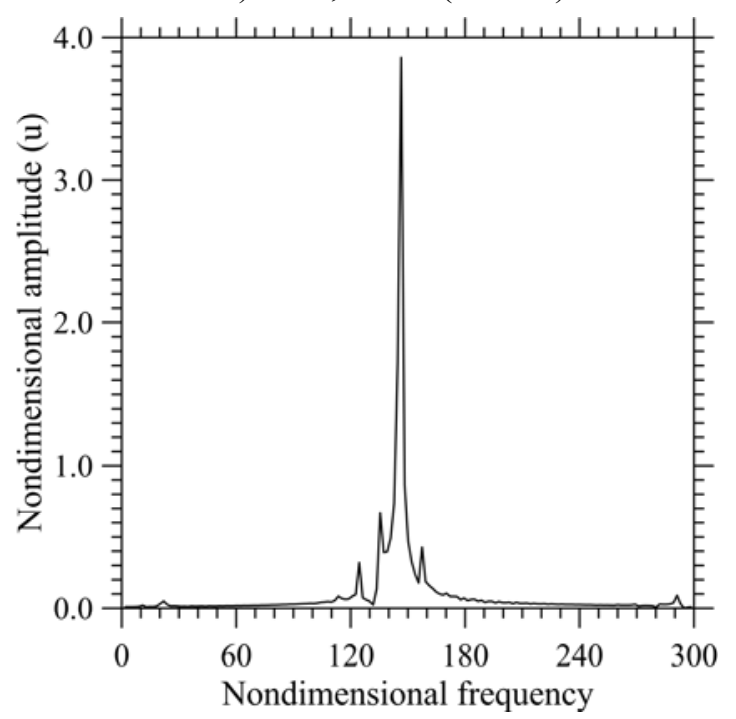

e) $15^{\circ} ; \operatorname{NOB}(\varepsilon=0.6)$

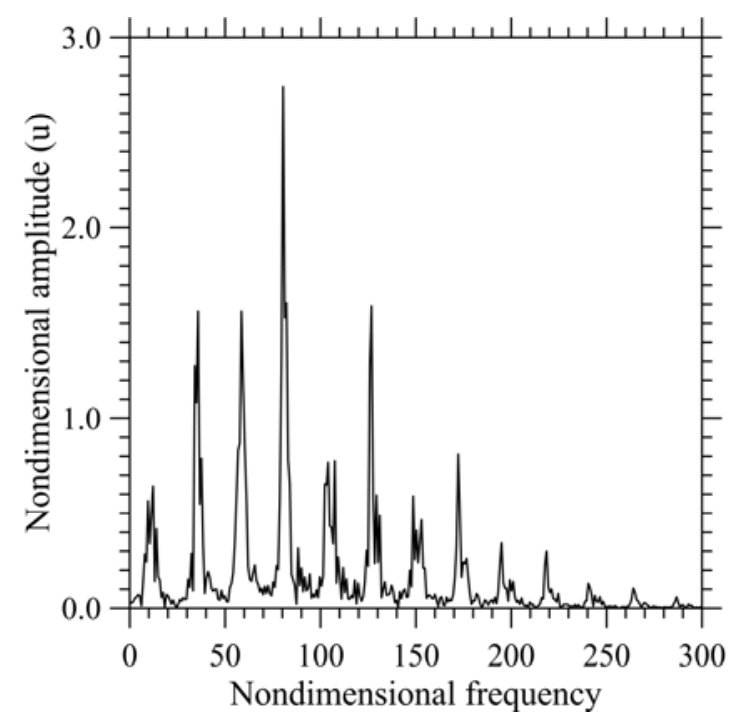

b) $-75^{\circ}$; Boussinesq

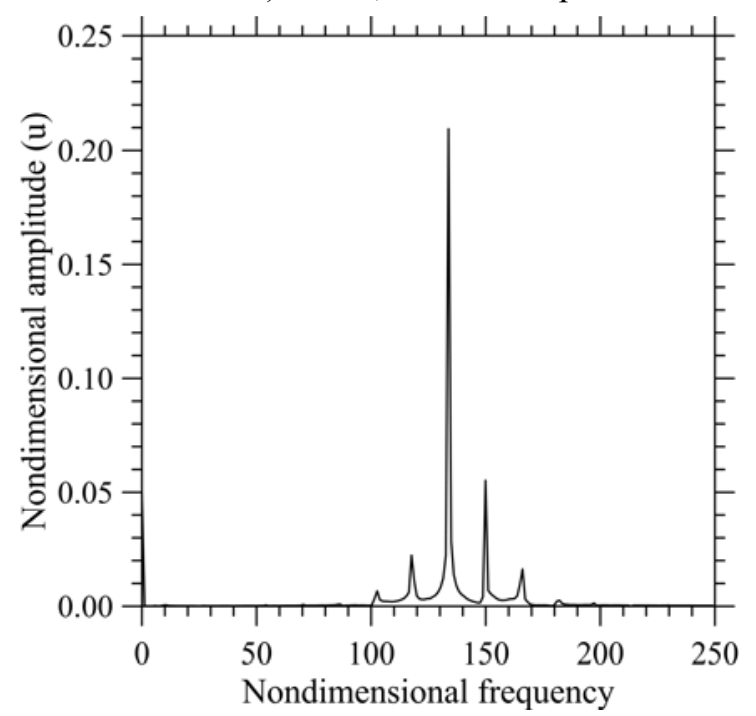

d) $-5^{\circ}$; Boussinesq

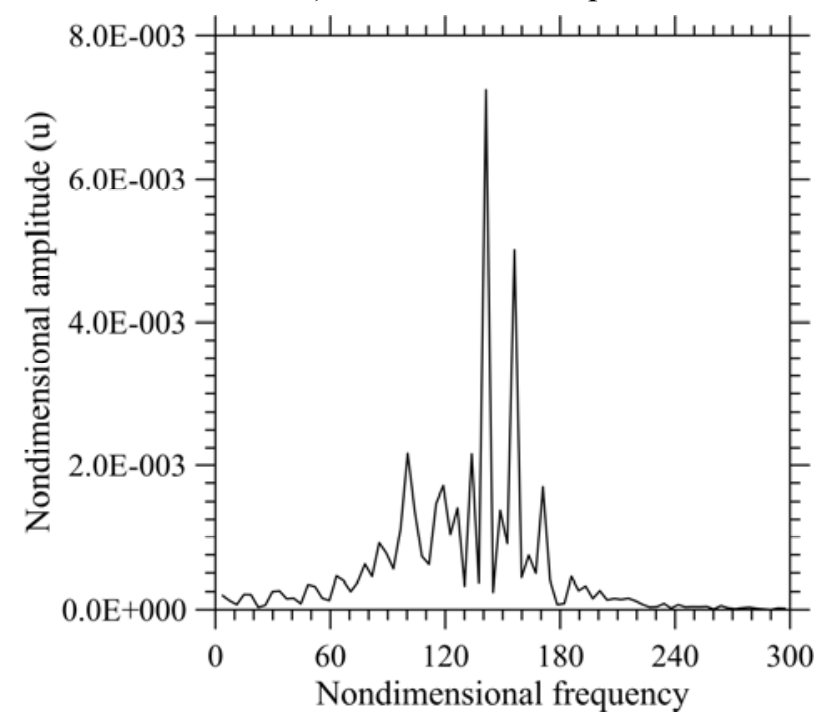

f) $15^{\circ}$; Boussinesq

Fig. 30: Examples of frequency spectra for $\mathrm{Ra}=3 \times 10^{5}$. 
When the Rayleigh number is increased to $\mathrm{Ra}=3 \times 10^{5}$, the most interesting phenomena occur in a given neighbourhood of the condition $\theta=0^{\circ}$ (Fig. 30). For negative values of $\theta$, the frequency spectra become much more complex when NOB effects are present. This difference is very evident for $\theta=-5^{\circ}$ (Figs. 30cd) and it tends to become less significant as $\theta$ tends to $\theta=-75^{\circ}$ (Fig. 30ab, for this value the spectra are rather similar from a qualitative point of view).

The flows are turbulent with one dominant frequency for $-30^{\circ} \leq \theta \leq 0^{\circ}$, and multi-modal for $\theta<-30^{\circ}$.

Following the same approach undertaken for $\mathrm{Ra}=1.9 \times 10^{5}$, additional simulations for an intermediate value of $\varepsilon(\varepsilon=0.2)$ and for the maximum considered value $\varepsilon=0.6$ (neglecting alternately the dependence of viscosity or density on temperature) have been used to identify the relative importance of the different effects causing the qualitative change from single frequency to the broadband state seen, e.g., for $\theta=-5^{\circ}$ (Figs. 30c and 30d). Along these lines, Fig. 31 clearly shows that, in this case, the broadband nature of the spectrum must be ascribed primarily to the joint effect of variable viscosity and variable density as when one of these two dependences is not taken into account, the spectrum becomes simpler (Fig. 32).

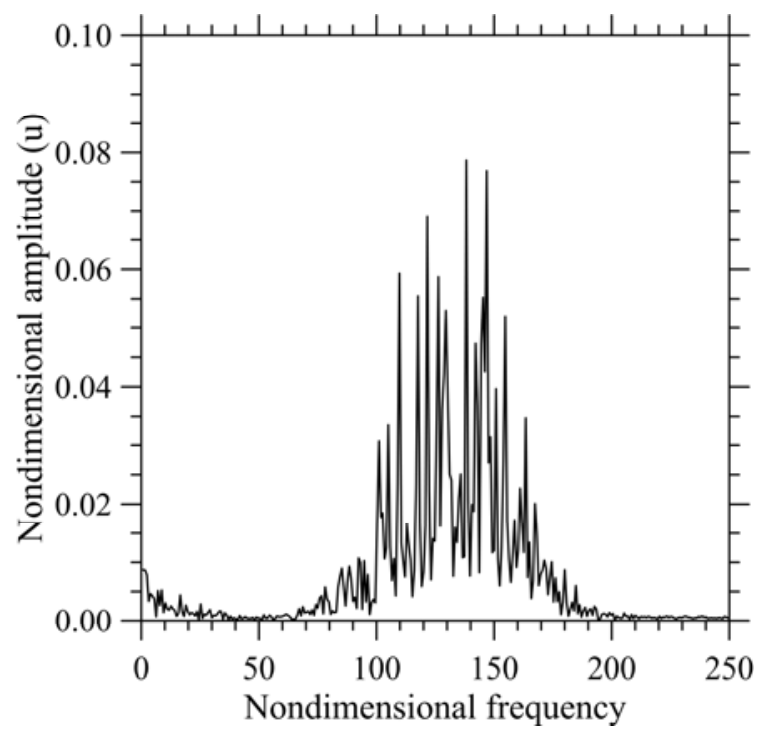

Fig. 31: Frequency spectra for $\mathrm{Ra}=3 \times 10^{5}, \varepsilon=0.2, \theta=-5^{\circ}$.

Such a trend, however, is reversed for $\theta=15^{\circ}$ (as the frequency spectrum is simpler in the NOB case, see Fig. 30ef). 

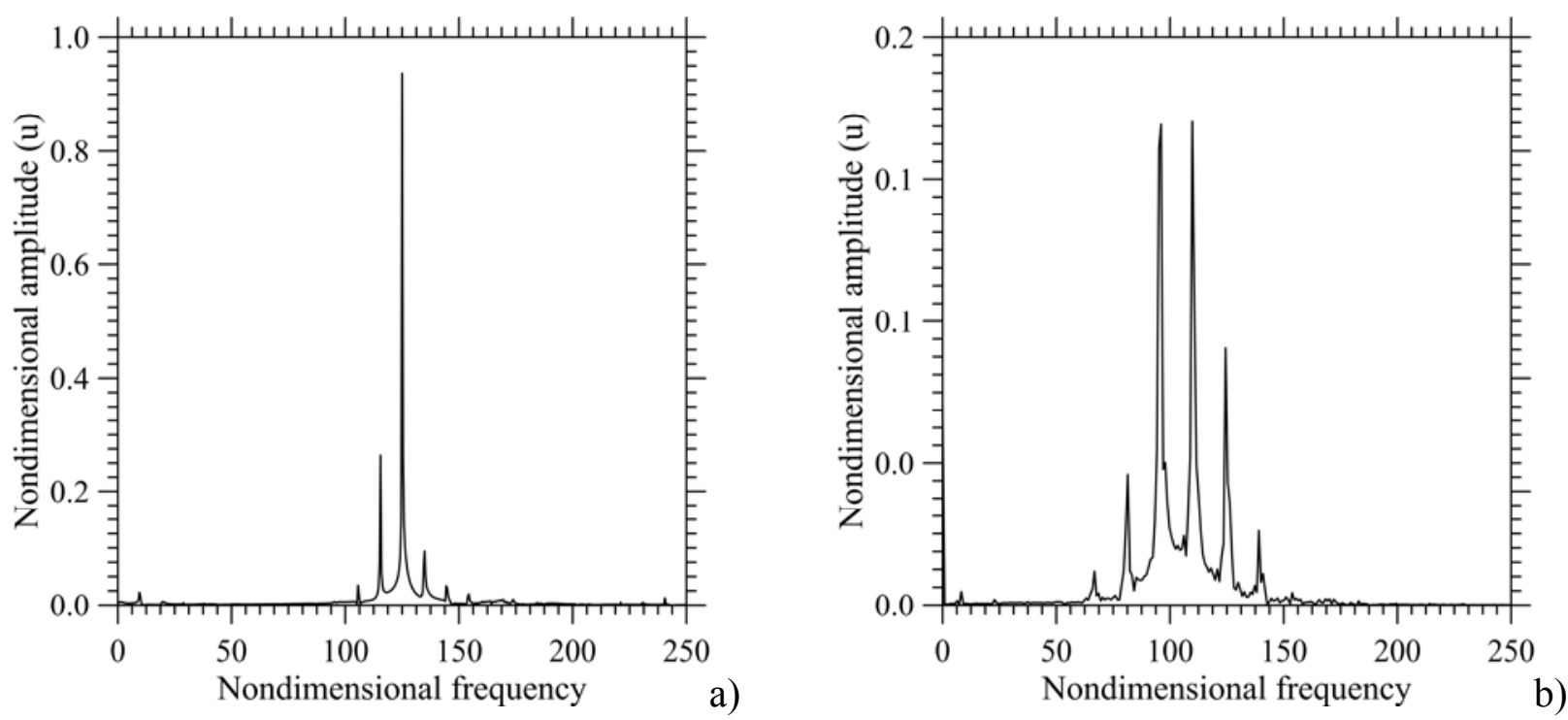

Fig. 32: Frequency spectra for $\mathrm{Ra}=3 \times 10^{5}, \varepsilon=0.6, \theta=-5^{\circ}$ and different models: a) Constant viscosity, variable density; b) Constant density, variable viscosity.

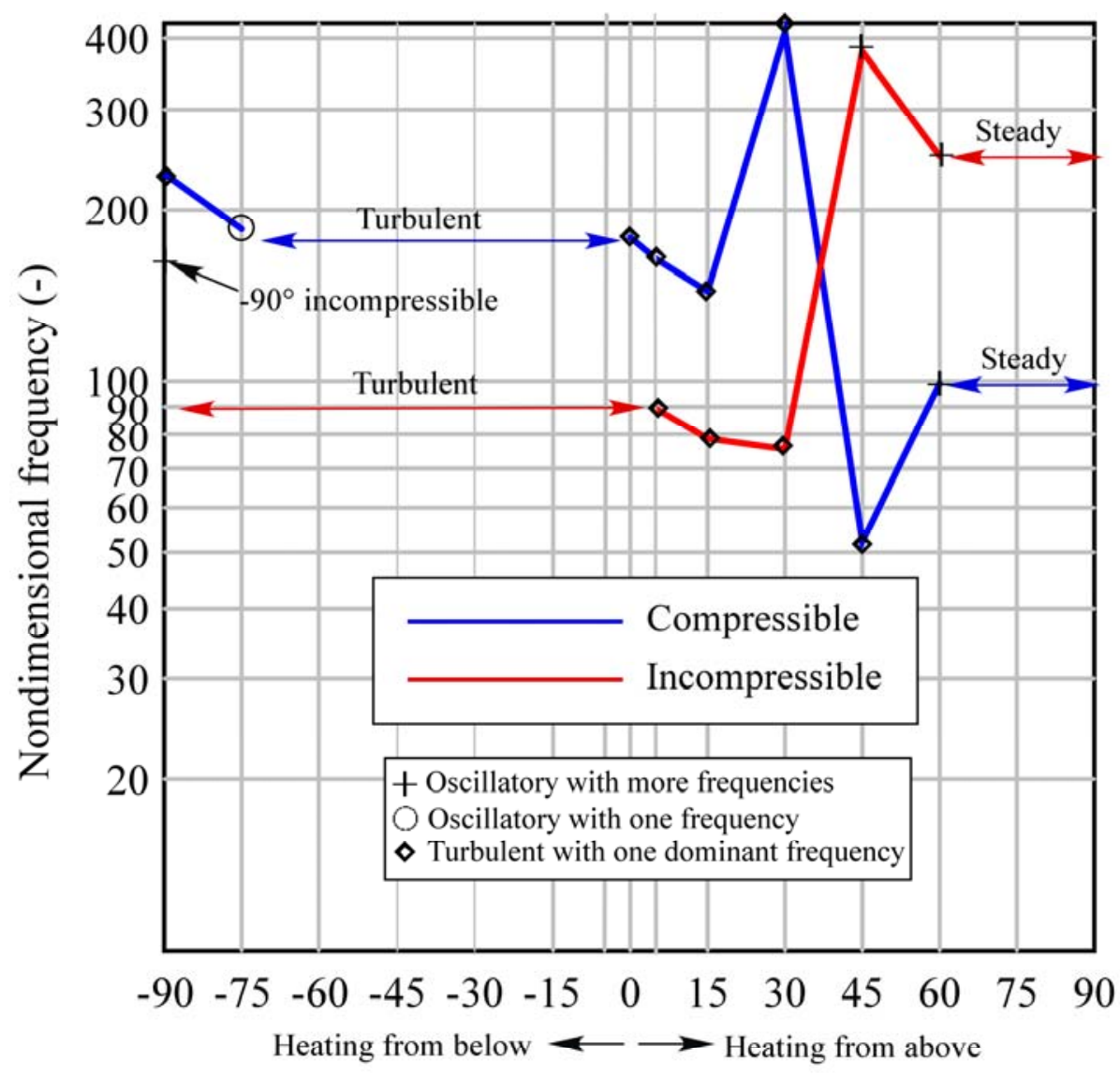

Inclination angle $\left(^{\circ}\right)$

Fig. 33: Frequency analysis for $\mathrm{Ra}=3 \times 10^{6}$ : comparison between incompressible and compressible ( $\varepsilon=0.6)$ flow solutions. 
Finally, for $\mathrm{Ra}=3 \times 10^{6}$ interesting dynamics can be seen when the inclination increases from $\theta=30^{\circ}$ to $\theta=45^{\circ}$ (Fig. 33). By comparing the frames for $\theta=45^{\circ}$ in Fig 34, it is evident that the peak with maximum amplitude (corresponding to the dominant frequency $f_{\mathrm{D}} \cong 380$ ) in the spectrum for the $\mathrm{OB}$ case (Fig. 34d) disappears when NOB effects are taken into account (with the dominant mechanism of oscillations being transferred to a much smaller frequency $f_{\mathrm{D}} \cong 50$ ).

Comparison of Figs. 34ab and 34cd also leads to another very interesting observation. A notable change can be also spotted in the general trend followed by the slopes of the turbulent spectra for $\theta=45^{\circ}$ and $\theta=-45^{\circ}$, respectively.

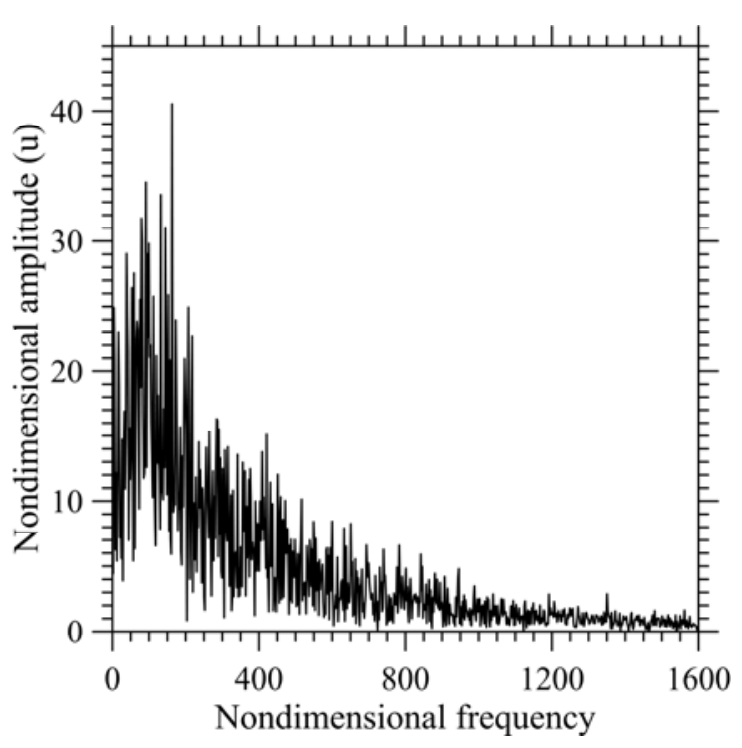

a) $-45^{\circ}$; $\mathrm{NOB}(\varepsilon=0.6)$

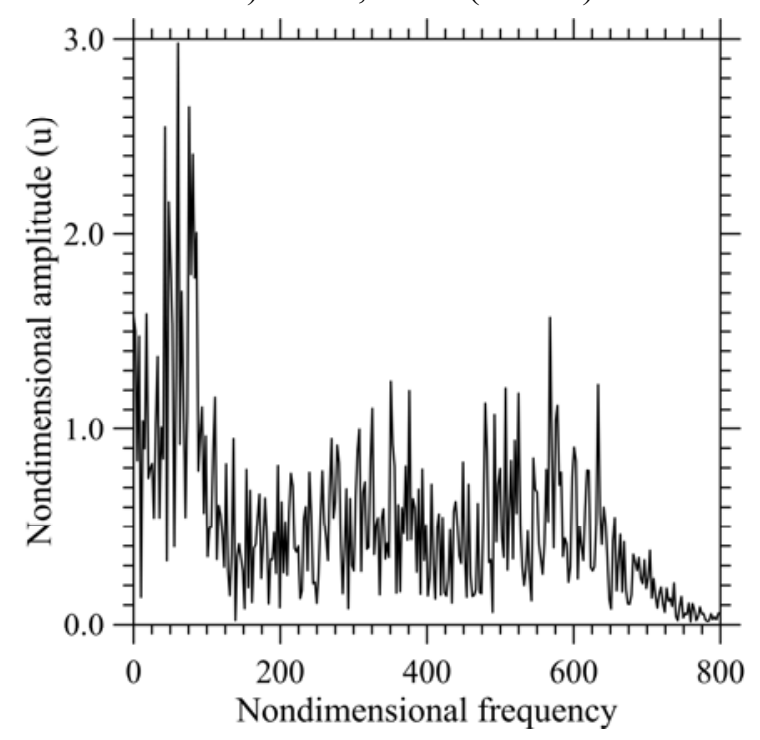

c) $45^{\circ} ; \mathrm{NOB}(\varepsilon=0.6)$

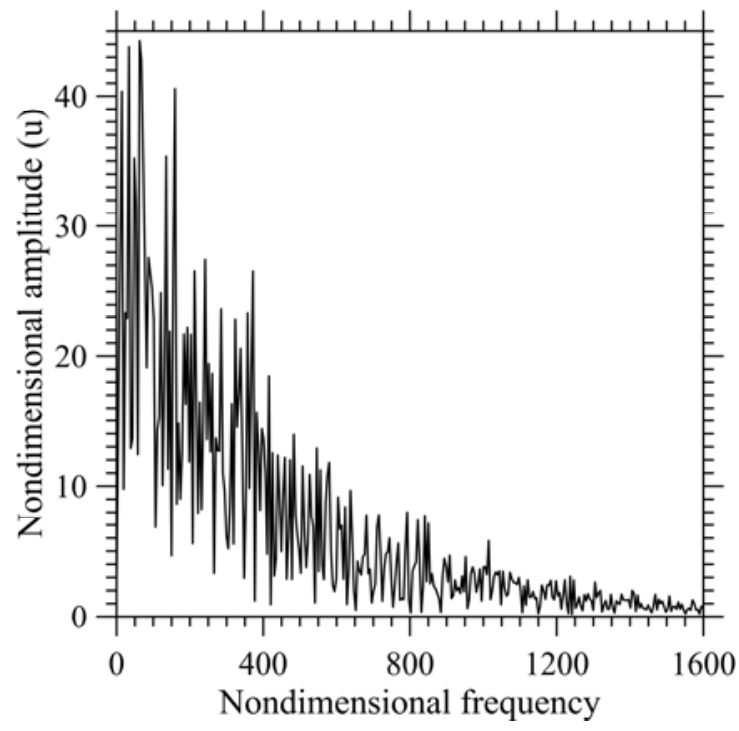

b) $-45^{\circ}$; Boussinesq

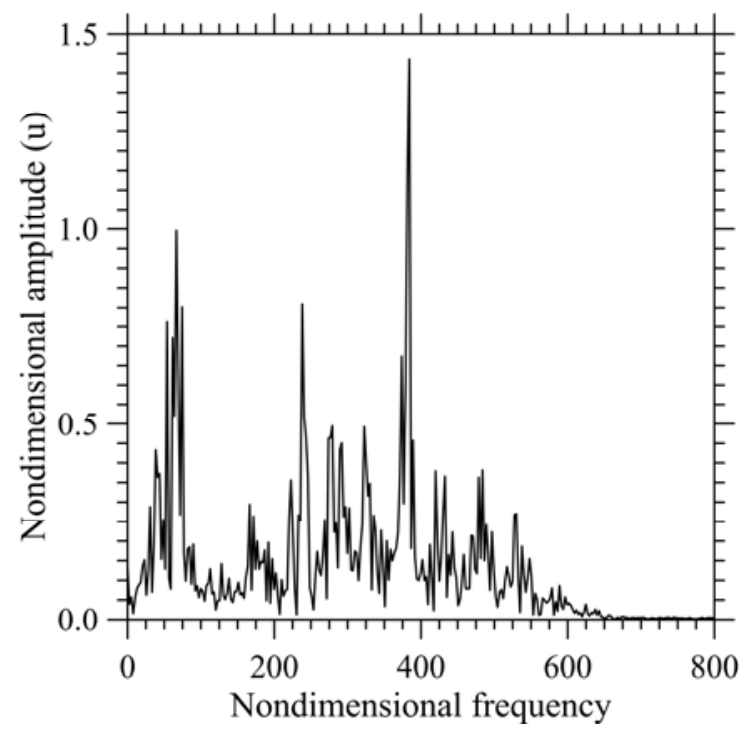

d) $45^{\circ}$; Boussinesq

Fig. 34: Examples of frequency spectra for $\mathrm{Ra}=3 \times 10^{6}$. 
Using the same procedure of De et al. [61], i.e. by plotting the frequency and related amplitude data using logarithmic scales for the axes and comparing the resulting plot with a power law $\mathrm{P}(f)=$ $\left(f \mid f_{\mathrm{c}}\right)^{-\mathrm{s}}$ where $f_{\mathrm{c}}$ is a fitting parameter, we have found that for $\theta=-45^{\circ}$ the frequency spectrum of velocities aligns perfectly with a $f^{-5 / 3}$ law in a wide band of frequencies (as implicit in the Kolmogorov similarity hypothesis, see Fig. 35a), whereas, for $\theta=45^{\circ}$ the exponent $\mathrm{s}$ is relatively close to 1.4 (see Fig. 35b; in agreement with earlier findings by Sano et al. [77], this may indicate that turbulence in the thermal convection may not be as simple as the theory proposed by Kolmogorov, especially when the temperature gradient has a heating-from-above stabilising vertical component).
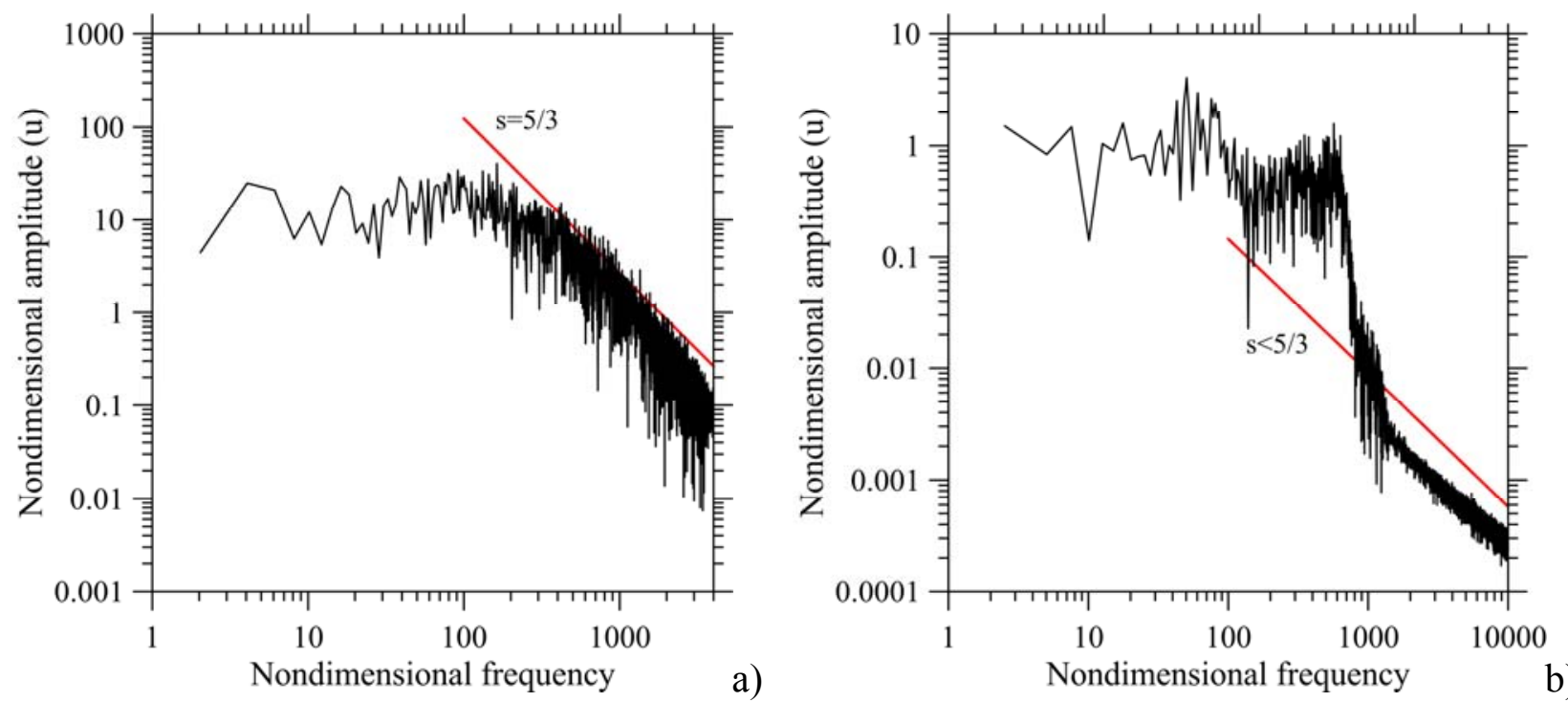

Fig. 35: Frequency spectra for $\mathrm{Ra}=3 \times 10^{6}, \varepsilon=0.6(\mathrm{NOB})$ : a) $\theta=-45^{\circ}$; b). $\theta=45^{\circ}$.

\section{Conclusions}

In general, the distinct modes of thermal (buoyancy) convection in a given geometric system can be delineated by considering various combinations of the possible symmetries along the horizontal and vertical directions. Remarkably, the existence of these symmetries implies the possibility of symmetry rupture, which is one of the fundamental concepts at the root of pattern formation phenomena in thermal convection. In the present work we have addressed the fundamental situation in which one or more of such symmetries can be broken due to fluid-dynamic instabilities occurring in the systems and/or because NOB (non-Boussinesq) effects are excited. The former are typically produced when a characteristic flow parameter (the Rayleigh number, $\mathrm{Ra}$, in our case) is sufficiently increased: the latter can become significant if a relatively large temperature difference is considered (regardless of the considered magnitude of the Rayleigh number).

Starting from the canonical case of a differentially heated slender vertical cavity, we have also examined the transversal role played in the above scenario by the presence of stabilizing or 
destabilizing components of buoyancy (according to the sign of the vertical component of the temperature gradient). This has been achieved by allowing the angle of inclination $\theta$ of the cavity with respect to the vertical direction to vary in space.

The results have shown that an interpretation of the variegated multitude of regimes produced by changes in Ra, $\theta$ and the intensity of NOB effects is not as straightforward as expected.

Starting from a canonical case (conditions for which the flow in the classical vertical enclosure $\left(\theta=0^{\circ}\right)$ with aspect ratio $A=8$ would be stable and steady in the OB limit and simply time-periodic in the variable-property framework, i.e. $\mathrm{Ra}=1.9 \times 10^{5}$ ), we have found that by inclining the axis of the cavity such that the heated wall goes below the cooled wall $\left(\theta<0^{\circ}\right)$, new oscillatory modes are excited. Such modes give rise to multi-modal (OMF) states for $-75^{\circ} \leq \theta \leq-5^{\circ}$ in the $\mathrm{OB}$ limit and simply periodic (OOF) regimes for $-45^{\circ} \leq \theta \leq-5^{\circ}$, when NOB effects are taken into account; interestingly, a further increase in the inclination in the latter case $\left(\theta \leq-60^{\circ}\right)$ can make the variableproperty flow steady.

Vice versa, when the cavity is inclined in such a way that the vertical component of buoyancy has a stabilizing role (heating from above), consideration of the NOB effects makes the flow oscillatory for $5^{\circ} \leq \theta \leq 15^{\circ}$, with steady conditions being recovered (regardless of the used model) for $\theta>15^{\circ}$. An increase in the Rayleigh number to $\mathrm{Ra}=3 \times 10^{5}$ has a remarkable impact on the NOB regimes of convection established for $\theta<0^{\circ}$ as witnessed by the changes experienced by the flows with timeperiodic behaviour seen for the smaller value of $\mathrm{Ra}$, which are taken over by relatively turbulent states. Similarly, for this value of $\mathrm{Ra}\left(3 \times 10^{5}\right)$, oscillatory regimes occupy the range of positive values of $\theta\left(5^{\circ} \leq \theta \leq 15\right)$ even if NOB effects are disregarded.

When the Rayleigh number is increased by an order of magnitude, as a feature common to all solutions, the complexity of the frequency spectrum increases significantly over the entire range of inclinations considered. An exception, however, is represented by the NOB regimes for $\theta \leq-60^{\circ}$, for which the flow retains a simply periodic (OOF) or multi-modal (OMF) behaviour. Interestingly, when the inclination is such that convective modes typical of RB convection enter the dynamics $(\theta<0)$, turbulence manifests in the frequency spectrum (the patterning behaviour still giving rise to recognizable structures), whereas if the temperature gradient has a vertical component corresponding to heating the system from above $(\theta>0)$, the complexity of the flow can be seen especially in terms of flow topology (featured by the emergence of many eddies).

A proper explanation for these fascinating trends must be sought in the non-trivial interplay between the typical modes of RB convection and those of the boundary-layer regime for the vertical cavity. The latter are much more sensitive to NOB effects (at least for the conditions considered in the present work) due to the significant thickening of the boundary layer on the hot side and the critical role played by disturbances growing in such boundary layers in the process leading to the onset of unsteadiness.

Future work shall be devoted to the exploration of regimes for which addressing the problem in the framework of direct numerical simulation would no longer be possible, with the ensuing need to implement adequate turbulent models (such as RANS or LES). 


\section{ACKNOWLEDGMENTS}

We would like to thank Hermes Ferialdi for the kind support provided in executing the additional simulations required to address the Reviewers' comments (and the related frequency spectrum analysis shown in Figures 27, 28, 31 and 32).

\section{References}

[01] Nield D.A., (1994), Convection induced by an inclined temperature gradient in a shallow horizontal layer, Int. J. Heat Fluid Flow, 15(2): 157-162.

[02] Delgado-Buscalioni R., Crespo del Arco E., Bontoux P., Ouazzani J., (1998), Convection and instabilities in differentially heated inclined shallow rectangular boxes, Compte Rend. Acad. Sci.. II B (Mechanics-Physics-Astronomy), 326(11): 711-718.

[03] Delgado-Buscalioni R. and Crespo del Arco E., (1999), Stability of thermally driven shear flows in long inclined cavities with end-to-end temperature gradient, Int. J. Heat Mass Transfer., 42: 2811-2822.

[04] Delgado-Buscalioni R. and Crespo del Arco E., (2001), Flow and heat transfer regimes in inclined differentially heated cavities, Int. J. Heat Mass Transfer, 44: 1947-1962.

[05] Baltasar J., Carvalho M.G., Coelho P., Costa m, (1997), Flue gas recirculation in a gas-fired laboratory furnace: Measurements and modelling, Fuel, 76(10): 919-929.

[06] von Backström T. W. and Gannon A.J., (2000), Compressible Flow Through Solar Power Plant Chimneys, J. Sol. Energy Eng., 122(3): 138-145.

[07] Elmo M, and Cioni O., (2003), Low Mach number model for compressible flows and application to HTR, Nuclear Engineering and Design, 222: 117-124.

[08] Hu S., Henager Jr. C. H., Heinisch H. L., Stan M., Baskes M. I., Valone S. M., (2009), Phasefield modeling of gas bubbles and thermal conductivity evolution in nuclear fuels, Journal of Nuclear Materials, 392(2): 292-300

[09] Martineau R. C., Berry R. A., Esteve A., Hamman K. D., Knoll D. A., Park R., Taitano W., (2010), Comparison of natural convection flows under VHTR type conditions modeled by both the conservation and incompressible forms of the Navier-Stokes equations, Nuclear Engineering and Design, 240: 1371-1385.

[10] Bensaci C.E., Labed A., Zellouf M., Moummi. A., (2017), Numerical study of natural convection in an inclined enclosure: application to flat plate solar collectors, Mathematical Modelling of Engineering Problems, 4(1): 1-6

[11] Lipps, F.B., (1971) Two-dimensional numerical experiments in thermal convention with vertical shear, J. Atm. Sci, 28, 3-19.

[12] Thorpe, S. A., (1987) Transitional phenomena and the development of turbulence in stratified fluids: A review, J. Geophys. Res. 92C, 5231-5248.

[13] Lappa M., (2009). Thermal Convection: Patterns, Evolution and Stability (John Wiley \& Sons, Chichester, England, 2009). 
[14] Vest C.M. and Arpaci V.S., (1969), Stability of natural convection in a vertical slot, J. Fluid Mech., 36: 1-15.

[15] Mizushima J. and Gotoh K., (1976), The stability of natural convection in a vertical fluid layer, J. Fluid Mech., 73 : 65-75.

[16] Bergholz R.F., (1978), Instabilities of steady natural convection in a vertical fluid layer, $J$. Fluid Mech., 84: 743-768.

[17] Elder J.W., (1965), Laminar free convection in a vertical slot, J. Fluid Mech., 23: 77-98.

[18] Seki N., Fukusako S., Inaba H., (1978), Visual observation of natural convective flow in a narrow vertical cavity, J. Fluid Mech., 84: 695-704.

[19] Chen C.F. and Thangam S., (1985), Convective stability of a variable viscosity fluid in a vertical slot, J. Fluid Mech.,161: 161-173.

[20] Chen F.L. and Wu C.H., (1993), Unsteady convection fllows in a vertical slot containing variable viscosity fluids, Int. J. Heat Mass Transfer, 36(17): 4233 -4246.

[21] Wakitani S., (1994), Experiments on convective instability of large Prandtl number fluids in a vertical slot, ASME J.Heat Transfer, 116: 120 -126.

[22] Roux B., Grondin J.C., Bontoux P., Davis G.D.V., (1980), Reverse transition from multicellular to monocellular motion in vertical fluid layer, Physico Chem Hydrodynam, 3(3F): 292-298.

[23] Lee Y. and Korpela S. A., (1983), Multicellular natural convection in a vertical slot, J. Fluid Mech. 126: 91-121.

[24] Chenoweth D. R. and Paolucci S., (1986), Natural convection in an enclosed vertical air layer with large horizontal temperature differences, J. Fluid Mech., 169: 173-210.

[25] Brenier B., Roux B., Bontoux P., (1986), Comparaison des méthodes tau-Chebyshev et Galerkin dans l'étude de la stabilité des mouvements de convection naturelle. Problème des valeurs propres parasites, J. Theorical. Appl. Mech., 5(1): 95-119.

[26] Le Quéré P., (1990), A note on multiple and unsteady solutions in twodimensional convection in a tall cavity, Trans. ASME, J. Heat Transfer 112: 965-974.

[27] Wakitani S., (1997), Development of multicellular solutions in natural convection in an airfilled vertical cavity, Trans. ASME, J. Heat Transfer 119: 97-101.

[28] Wakitani S., (1998), Flow patterns of natural convection in an air-filled vertical cavity, Phys. Fluids, 10(8): 1924-1928.

[29] Christon M.A., Gresho P.M., Sutton S.B., (2002), Computational predictability of timedependent natural convection flows in enclosures (including a benchmark solution), Int. J. Numer. Methods Fluids, 40: 953-980.

[30] Le Quéré P., Masson R., Perrot P., (1992), A Chebyshev collocation algorithm for 2D nonBoussinesq convection, J. Comput. Phys., 103: 320-335.

[31] Weisman C., Calsyn L., Dubois C., Le Quéré P., (2001), Sur la nature de la transition à l'instationnaire d'un écoulement de convection naturelle en cavité différentiellement chauffée à grands écarts de température. Compte Rendu de l'Académie des Sci, 329(Série II b): 343-350.

[32] Bouloumou O., Serre E., Bontoux P., Fröhlich J., (2012), A 3D pseudo-spectral low Machnumber solver for buoyancy driven flows with large temperature differences, Computers \& Fluids, 66: 107-120. 
[33] Ortiz-Pérez A. S., and Dávalos-Orozco L. A., (2011), Convection in a horizontal fluid layer under an inclined temperature gradient, Phys. Fluids., 23, 084107.

[34] Ortiz-Pérez A. S., and Dávalos-Orozco L. A., (2015), Convection in a horizontal fluid layer under an inclined temperature gradient with a negative vertical Rayleigh number, Int. J. Heat Mass Transfer, 90: 1214-1220.

[35] Saury D., Benkhelifa A., Penot F., (2012), Experimental determination of first bifurcations to unsteady natural convection in a differentially-heated cavity tilted from 0 to 180, Exper. Therm. Fluid Sci. 38: 74-84.

[36] Torres J. F., Henry D., Komiya A., and Maruyama S., (2015), Transition from multiplicity to singularity of steady natural convection in a tilted cubical enclosure, Phys. Rev. E, 92(2), 023031.

[37] Williamson N., Armfield S.W., Lin W., Kirkpatrick M.P., (2016), Stability and Nusselt number scaling for inclined differentially heated cavity flow, Int. J. Heat Mass Transfer, 97: 787-793.

[38] Lappa M., (2016), A Mathematical and Numerical Framework for the Analysis of Compressible Thermal Convection in Gases at very high Temperatures, J. Comput. Phys., 313 : 687-712.

[39] Oberbeck A., (1879), Ueber die Wärmeleitung der Flüssigkeiten bei Berücksichtigung der Strömungen infolge von Temperaturdifferenzen, Ann. Phys. Chem., 7: 271-292.

[40] Boussinesq J., (1903), Théorie analytique de la chaleur, mise en harmonie avec la thermodynamique et avec la théorie mécanique de la lumière, Vol. 2, Paris, Gauthier-Villars, 1901-1903.

[41] Gray D. and Giorgini A., (1976), The validity of the Boussinesq approximation for liquids and gases, Heat and Mass Transfer, 15: 545-551.

[42] Paolucci S., (1982), On the filtering of sound from the Navier-Stokes equations, Technical Report SAND 82-8251, Sandia National Laboratories, Livermore, 1982.

[43] Müller B., (1998), Low Mach number asymptotics of the Navier-Stokes equations, J. Eng. Math. 34(1-2), 97-109.

[44] Munz C.-D., Roller S., Klein R., Geratz K.J., (2003), The extension of incompressible flow solvers to the weakly compressible regime, Computers \& Fluids, 32(2): 173-196.

[45] Beccantini A., Studer E., Gounand S., Magnaud J.-P., Kloczko T., Corre C. and Kudriakov S., (2008), Numerical simulations of transient injection flow at low Mach number regime, Int. J. Numer. Meth. Engng, 76: 662-696.

[46] Benteboula S. and Lauriat G., (2009), Numerical simulations of anisothermal laminar vortex rings with large density variations, Int. J. Heat \& Fluid Flow, 30: 186-197.

[47] Jang D.S., Jetli R., Acharya S. (1986), Comparison of the PISO, SIMPLER and SIMPLEC algorithms for the treatment of the pressure-velocity coupling in steady flow problems, Numer Heat Transfer 10: 209-228.

[48] Moukalled F., Mangani L. and Darwish M., (2016), The Finite Volume Method in Computational Fluid Dynamics - An Advanced Introduction with OpenFOAM and Matlab, Springer International Publishing, 2016, New York). 
[49] Rhie C.M. and Chow W.L., (1983), Numerical study of the turbulent flow past an airfoil with trailing edge separation. AIAA J 21:1525-1532.

[50] Choi SK, Nam HY, Cho M (1994) Systematic comparison of finite-volume calculation methods with staggered and nonstaggered grid arrangements. Numer Heat Transfer, Part B 25 (2): 205-221

[51] Choi SK, Nam HY, Cho M (1994) Use of staggered and nonstaggered grid arrangements for incompressible flow calculations on nonorthogonal grids. Numer Heat Transfer, Part B 25(2): 193-204.

[52] Paillère H. and Le Quéré P., (2000) Modelling and simulation of natural convection flows with large temperature differences: a benchmark problem for low Mach number solvers, 12th Séminaire de Mécanique des Fluides Numérique, CEA Saclay, France, 25-26 Jan., 2000.

[53] CEA/Nuclear Reactor Division, Numerical Workshop, January 25-27, 2000. Modeling and simulation of natural flows with large temperature differences: a benchmark problem for low Mach number solvers. In: 12th Seminar "Computational Fluid Dynamics", INSTN Saclay, France.

[54] INRIA and MAB, Numerical Workshop, June 21-25, 2004. Mathematical and Numerical Aspects of Low Mach Number Flows. Porquerolles, France.

[55] Paillère H., Le Quéré P., Weisman C., Vierendeels J., Dick E., Braack M., Dabbene F., Beccantini A., Studer E., Kloczko T., Corre C., Heuveline V., Darbandi M., and Hosseinizadeh S.F., (2005), Modelling of natural convection flows with large temperature differences: A benchmark problem for low Mach number solvers. Part 2. contributions to the june 2004 conference, ESAIM: Mathematical Modelling and Numerical Analysis, 39(3): 617-621.

[56] Paolucci S., (1990), Direct numerical simulation of two-dimensional turbulent natural convection in an enclosed cavity, J. Fluid Mech., 215, 229-262.

[57] Farhangnia M., Biringen S, Peltier L. J., (1996), Numerical Simulation of Two-dimensional Buoyancy-driven Turbulence in a Tall Rectangular Cavity, Int. J. Numer. Meth. Fluids, 23(12): 1311 - 1326.

[58] Trias F.X., Soria M., Oliva A. and Pérez-Segarra C.D, (2007), Direct numerical simulations of two- and three-dimensional turbulent natural convection flows in a differentially heated cavity of aspect ratio 4, J. Fluid Mech., 586: 259-293.

[59] Shishkina O., Stevens R.J.A.M., Grossmann S. and Lohse D., (2010), Boundary layer structure in turbulent thermal convection and its consequences for the required numerical resolution, New J. Phys. 12, 075022 (17 pages).

[60] Kerr R.M., (1996), Rayleigh number scaling in numerical convection, J. Fluid Mech. 310, 139179.

[61] De A.K., Eswaran V., Mishra P.K., (2017), Scalings of heat transport and energy spectra of turbulent Rayleigh-Bénard convection in a large-aspect-ratio box, Int. J. Heat Fluid Flow, 67, 111-124.

[62] Scheel J., Kim E., and White K., (2012), Thermal and viscous boundary layers in turbulent Rayleigh-Bénard convection, J. Fluid Mech., 711, 281-305. 
[63] Lappa M., (2011), Some considerations about the symmetry and evolution of chaotic Rayleigh-Bénard convection: the flywheel mechanism and the "wind" of turbulence, $C . R$., Méc. 339: 563-572.

[64] Altaç Z. and Ugurlubilek N., (2016), Assessment of turbulence models in natural convection from two- and three-dimensional rectangular enclosures, Int. J. Therm. Sci., 107: 237-246.

[65] Mason P. J., (1994), Large-Eddy Simulation of the Convective Atmospheric Boundary Layer, J. Atmos. Sci. 46, 1492-1516.

[66] Kimmel S.J. and Domaradzki J. A., (2000), Large eddy simulations of Rayleigh-Bénard convection using subgrid scale estimation model, Phys. Fluids 12, 169-184.

[67] Salat J., Xin S., Joubert P., Sergent A., Penot F., Le Quere P., (2004), Experimental and numerical investigation of turbulent natural convection in a large air-filled cavity, Int. J. Heat Fluid Flow, 25: 824-832.

[68] Le Quéré P., Weisman C., Paillère H., Vierendeels J., Dick E., Becker R., Braack M., Locke J., (2005), Modelling of natural convection flows with large temperature differences: a benchmark problem for low Mach number solvers. Part 1: reference solutions, Mathematical Modelling and Numerical Analysis, 39(3):609-616.

[69] Salinger A. G., Lehoucq R. B., Pawlowski R. P. and Shadid J. N., (2002), Computational bifurcation and stability studies of the 8:1 thermal cavity problem, Int. J. Numer. Meth. Fluids, 40(8): 1059-1073.

[70] Zhang J., Childress S., and Libchaber A., (1997), Non-Boussinesq effect: Thermal convection with broken symmetry. Phys. Fluids, 9(4), 1034-1042.

[71] Robinson F. and Chan K., (2004), Non-Boussinesq simulations of Rayleigh-Bénard convection in a perfect gas, Phys. Fluids, 16(5): 1321-1333.

[72] Chen Y. and Pearlstein A.J., (1989), Stability of free-convection flows of variable-viscosity fluids in vertical and inclined slots, J. Fluid Mech., 198: 513-541.

[73] Fujimura K. and Kelly R.E., (1993), Mixed mode convection in an inclined slot, J. Fluid Mech., 246: 545-568.

[74] Kaloni P.N. and Qiao N., (1996), On the nonlinear stability of thermally driven shear flow heated from below, Phys. Fluids, 8: 639-641.

[75] Goldhirsch I., Pelz R.B. and Orszag S.A., (1989), Numerical simulation of thermal convection in a two-dimensional finite box, J. Fluid Mech., 199: 1-28

[76] Bouabdallah S., Ghernaout B., Teggar M., Benchatti A. and Benarab F.Z., (2016), Onset of Natural Convection and Transition Laminar-Oscillatory Convection Flow in Rayleigh-Bénard Configuration, Int. J. Heat Technol., 34(1): 151-157, DOI: 10.18280/ijht.340122

[77] Sano M. , Wu X.Z. , Libchaber A. , (1989), Turbulence in helium-gas free convection, Physical Review A, 40(11), 6421-6430. 